\title{
Dihedral symmetries of multiple polylogarithms
}

\author{
Susama AgarWala
}

This paper finds relationships between multiple polylogarithms with a dihedral group action on the arguments. I generalize the combinatorics developed in Gangl, Goncharov and Levin's $R$-deco polygon representation of multiple polylogarithms to find these relations. These relations between $R$-deco polygons, and between $R$-deco polygons and iterated integrals, can only be defined up to a primitive co-ideal.

1. The various Hopf algebras of $R$-deco polygons

1.1. Bar constructions on $\boldsymbol{R}$-deco polygons

1.2. Multi-rooted trees

1.3. Hopf algebras of trees associated to dissected polygons

1.4. From generating sets to differentials

1.5. Properties of almost compatible algebras

2. Permutations of a polygon

2.1. Order 2 generator of the dihedral group

2.2. Order $n$ generator of the dihedral group

2.2.1 Relating $B_{\phi_{2}}$ to $B_{\phi_{4}}$

2.2.2 Introducing a new symmetry. 
This paper studies the relationships between multiple polylogarithms that differ by a dihedral permutation on their arguments. Goncharov has shown [8] dihedral relations between multiple zeta values, and has conjectured such a relation on the level of multiple polylogarithms. To study the dihedral symmetries of multiple polylogarithms, I use a technique developed by Gangl et al. [7], that represents multiple polylogarithms as decorated rooted oriented polygons, $R$-deco polygons. Goncharov [9] has shown a Hopf algebra structure underlying multiple polylogarithms. The authors of [7] give a Hopf algebra structure to the $R$-deco polygons and construct a coalgebra homomorphism between their Hopf algebra of polygons and the Hopf algebra of multiple polylogarithms. The $R$-deco polygon structure of multiple polygons has recently become interesting objects in physics. These polygons are used to define symbols of multiple polylogarithms [6]. These symbols and their Hopf algebraic structure have important applications in understanding amplitudes in super Yang-Mills theories in 4 dimensions [5, 11].

Multiple polylogarithms are interesting number theoretical objects in their own right. As a nested sum they are written

$$
\mathbb{L i}_{n_{1}, \ldots, n_{r}}\left(x_{1}, \ldots, x_{r}\right)=\sum_{0<k_{1}<\ldots<k_{r}} \frac{x_{1}^{k_{1}}, \ldots, x_{r}^{k_{r}}}{k_{1}^{n_{1}}, \ldots, k_{r}^{n_{r}}}
$$

The multiple polylogarithm, evaluated at $x_{i}=1$ gives the multiple zeta value $\zeta\left(n_{1}, \ldots, n_{r}\right)$. The weight of the multiple polylogarithm is $w=\sum_{i=1}^{r} n_{i}$ and its depth is $r$. In [9], this multiple sum is written in terms of Chen's iterated integrals. Thus multiple polylogarithms inherit the bar complex defined on iterated integrals [3]. There is a motivic generalization of Chen's iterated integrals. Bloch and Kriz [1] define a Hopf algebra of algebraic cycles, $\chi_{\text {Mot }}$, over a field $F$ formed by taking the 0th cohomology of a bar complex based on a differential graded algebra (DGA) associated to the cycles. In [7], the authors determine that there are elements of $\chi_{\text {Mot }}$ that correspond to multiple polylogarithms.

Iterated integrals also make their appearance in physics. Goncharov [10] shows a relationship between the Hopf algebra of multiple polylogarithms, via iterated integrals, and the Hopf algebra of rooted trees developed by Connes and Kreimer for renormalizing Feynman integrals [4]. Brown [2] develops a method for evaluating Feynman integrals, under Schwinger parametrization, in terms of iterated integrals, although the arguments for these iterated integrals are more complicated than those for multiple polylogs. In short, there is a lot of activity suggesting a close tie between multiple 
polylogarithms and the calculations that appear in various quantum field theories.

This paper studies multiple polylogarithms from a combinatorial point of view, primarily on the level of $R$-deco polygons. Let $D_{2 r}$ be the dihedral group on $r$ elements,

$$
D_{2 r}=\left\langle\sigma, \tau \mid \tau^{2}=\sigma^{r}=1, \sigma \tau=\tau \sigma^{-1}\right\rangle
$$

In this paper, I study the relationship between the multiple polylogarithms

$$
\mathbb{L i}_{n_{1}, \ldots, n_{r}}\left(x_{1}, \ldots, x_{r}\right) \text { and } \operatorname{Li}_{g\left(n_{1}, \ldots, n_{r}\right)}\left(g\left(x_{1}, \ldots, x_{r}\right)\right)
$$

for any $g \in D_{2 r}$. Section one of this paper generalizes the algorithm defined in [7] for assigning decorated trees to multiple polylogarithms. There the authors associate to each multiple polylogarithm an $R$-deco polygon, and a family of dissections to each polygon. The differential structure of the iterated integral associated to each multiple polylogarithm defines a dual tree structure to the dissections, and a Hopf algebra structure to the $R$ - deco polygons. Each polygon is mapped to an element of the bar complex on the algebra of $R$-deco polygons, $\mathcal{P}_{\bullet}^{(\star)}(R)$. I introduce a generalization of a rooted tree, called a multi-rooted tree. I show that if the dual trees associated to dissections generate a Hopf algebra, the trees dual to single dissections define a differential on the $\mathcal{P}_{\bullet}^{(\star)}(R)$. Combinatorial relationships between these different tree structures allow me to compare multiple polylogarithms of weight $r$ under the action of the dihedral group $D_{2 r}$. Section 2 of this paper calculates the action of $\tau$ and $\sigma$ in $D_{2 r}$ on $\mathcal{P}_{\bullet}^{(\star)}(R)$. Contrary to a conjecture by Gangl and Levin, I find that the difference between the bar element associated to an $R$-deco polygon and its image under the action of the dihedral group cannot completely be expressed as a sum of products of bar elements associated to subpolygons. On the level of $R$-deco polygons, this result holds up to a primitive coideal.

\section{The various Hopf algebras of $R$-deco polygons}

This paper studies multiple polylogarithms by studying the iterated integral associated to them. Let $w=\sum_{i}^{n} k_{i}$ be the weight of the iterated integral

$$
\begin{aligned}
& I_{k_{1}, \ldots, k_{n}}(\gamma)\left(0 ; x_{1}, \ldots, x_{n} ; x_{n+1}\right) \\
& \quad=\int_{\gamma} \frac{d t_{1}}{t_{1}-x_{1}} \wedge \bigwedge_{i=1}^{k_{1}-1} \frac{d t_{1_{i}}}{t_{1_{i}}} \cdots \wedge \frac{d t_{n}}{t_{n}-x_{n}} \bigwedge_{i=1}^{k_{n}-1} \frac{d t_{n_{i}}}{t_{n_{i}}}
\end{aligned}
$$


with $\gamma$ a path in $\mathbb{C}$ such that $\gamma(0)=0, \gamma(1)=x_{n+1}$. The value of this integral depends on the homotopy class of $\gamma$ [3]. If $\gamma$ is a straight path in the real line, $\gamma(t) \in \mathbb{R}$, from $x_{i}$ to $x_{i+1} \in \mathbb{R}$ I drop the notation $\gamma$. These iterated integrals can be related to multiple logarithms

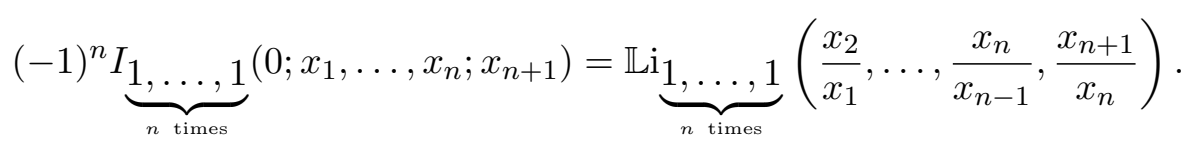

These iterated integrals live in the 0th cohomology of the associated bar complex defined by Chen. The general class of iterated integrals, $I_{\bullet}(R)$ have a Hopf algebra structure, as shown by Goncharov in [8]. The space of iterated integrals can be endowed with an algebra structure, under path composition. In [10], the author further shows that these iterated integrals have a motivic counterpart, $I^{\mathcal{M}}\left(0, x_{1}, \ldots, x_{n+1}\right)$ with $x_{i} \in F$, for a field $F$, that is an element of the fundamental motivic Hopf algebra over $F$. The natural differential on the iterated integrals defines a bar complex on algebra of iterated integrals. Those iterated integrals corresponding to multiple polylogarithms live in the 0th cocycle of this bar complex.

In [7], Gangl et al. associate to each multiple polylogarithm an $R$-deco polygon. For instance, the integral

$$
\underbrace{I_{1, \ldots, 1}}_{n \text { times }}\left(0 ; x_{1}, \ldots, x_{n} ; x_{n+1}\right)
$$

is associated to an oriented $n+1$-gon with sides labeled from $x_{1}$ to $x_{n+1}$. Note that in this case, $x_{i} \neq 0$ by construction. The authors define a differential on the algebra of $R$-deco polygons, that mimics the differential on iterated integrals. This defines a bar complex on $R$-deco polygons. The authors of loc. cit. associate a bar element to each polygon. They define a family of dual trees to the $R$-deco polygons which induce a Hopf algebra structure on the algebra of $R$-deco polygons. This translates to a Hopf algebra sitting in the the 0th cocycle of the bar complex of $R$-deco polygons. In this paper, I call this $B_{\phi_{2}}$. This structure on the $R$-deco polygons is compatible with the parallel structure on iterated integrals in that there is a coalgebra homomorphism from this to $I_{\bullet}(R)$,

$$
\Phi: \Lambda\left(B_{\phi_{2}}\right) \rightarrow I_{\bullet}(R) .
$$


Specifically, if $0 \notin R$, and the polygon $P$ has sides labeled $\left\{x_{1}, \ldots, x_{n+1}\right\}$ then

$$
\Phi\left(\mathfrak{T}_{\phi_{2}}(P)\right)=\underbrace{I_{1, \ldots, 1}}_{n \text { times }}\left(0 ; x_{1}, \ldots, x_{n} ; x_{n+1}\right) .
$$

Relating dihedral symmetries of multiple polylogarithms can be simplified to a combinatorial problem on the dihedral symmetries of decorated polygons.

This section generalizes the above construction. I define a class of Hopf algebras associated to these decorated polygons that are useful in solving the combinatorics of how polylogarithms vary as their order of the arguments are changed. Section 1.1 gives a definition of $R$-deco polygons and their dissections, the vector space they generate, $V_{\bullet}(R)$, its exterior product algebra $\mathcal{P}_{\bullet}^{(\star)}(R)$, and the associated bar complexes. Section 1.2 defines the Hopf algebra of multi-rooted trees, $\mathcal{T}^{\bullet}(R)$, and the linearization map $\Lambda$. I show that $\Lambda$ is a bialgebra homomorphism from $\mathcal{T}^{\bullet}(R)$ to the commutative Hopf algebra of words on $R$-deco polygons. Section 1.3 introduces a family of dissection compatible Hopf algebras of $\mathcal{T}^{\bullet}(R)$ generated by sets associated to dissections of $R$-deco polygons. Section 1.4 , shows that these generating sets define differentials on $\mathcal{P}_{\bullet}^{(\star)}(R)$. It also introduces a family of almost compatible algebras. Finally, Section 1.5 defines a relationship between the linearizations of dissection compatible Hopf algebras and almost compatible algebras that is useful for the calculations in Section 2. Furthermore, I show that the linearization of the latter also gives rise a Hopf algebra.

\subsection{Bar constructions on $R$-deco polygons}

Let $R$ be a set. Usually, $R$ is the set underlying a field.

Definition 1. Let $P_{n}$ be the convex oriented polygon with $n+1 \geq 2$ sides, with sides labeled by elements in $R$. One of those sides is a distinguished side, called a root side. One of the endpoints of the root side is marked as the first vertex. Orient $P_{n}$ by starting at the first vertex and ending at the root side. The polygon $P_{n}$ is an $R$-deco polygon, as defined in [7].

In this paper, I draw polygons to be oriented counterclockwise. I sometimes specify a polygon in terms of its labels, proceeding counterclockwise 
and ending with the root side. Therefore,

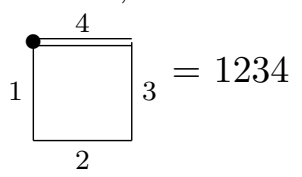

The $R$-deco polygons generate a vector space.

Definition 2. Let $V_{\bullet}(R)$ be the graded vector space over $\mathbb{Q}$ generated by $R$-deco polygons. Let $V_{n}(R)$ be the vector space over $\mathbb{Q}$ generated by $R$-deco $n+1$-gons, with $n \geq 0$ and $V_{0}(R)$ identified with $\mathbb{Q}$

$V_{\bullet}(R)=\mathbb{Q}<\{1, P \mid P$ is an $R$-deco polygon $\}>=\oplus_{n=0}^{\infty} V_{n}(R) ; \quad V_{0}(R)=\mathbb{Q}$.

The 'weight' of an element in $V_{n}(R)$ is $n$.

An $R$-deco polygon can be equipped with arrows, as in [7]. An arrow of a polygon is drawn from a vertex of a polygon to a side of a polygon. It divides the interior of the polygon into two regions. A trivial arrow of a polygon $P$ goes from a vertex to an adjacent side. A non-trivial arrow of $P$ is an arrow that does not end on a side adjacent to its starting vertex. Two arrows are said to be non-intersecting if they share no points in common other than possibly the starting vertex.

Definition 3. Arrows of $P_{n}$ are defined by their starting vertex and ending edge. Write ${ }_{i} \alpha_{j}$ for an dissecting arrow of the polygons $P$ that starts at the $i$ th vertex and ends on the $j$ th edge. For non-trivial arrows, $j \neq i, i-1$ $\bmod (n+1)$. Call ${ }_{i} \alpha_{j}$ a backwards arrow if $j<i$. Otherwise it is a forwards arrow.

Example 4. The arrow ${ }_{1} \alpha_{4}$ is a trivial arrow in the first polygon below. In the second polygon, ${ }_{2} \alpha_{4}$ and ${ }_{4} \alpha_{2}$ are non-intersecting, non-trivial arrows.
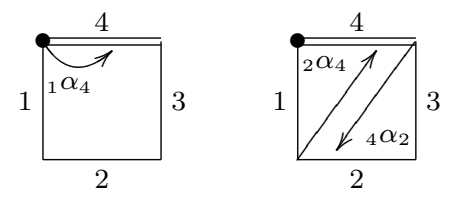

Regions associated to dissection arrows can be viewed as polygons in their own right. If $\alpha$, is an arrow of $P$, contracting $\alpha$ to a point results in a set of two polygons $\left\{P_{\alpha}, Q_{\alpha}\right\}$ associated to the two regions of $P$ as follows.

(1) The labels of the sides and the orientations of $P_{\alpha}$ and $Q_{\alpha}$ are inherited from $P$. 
(2) If $\alpha$ lands on a non-root side of $P$, then the subpolygon corresponding to the region that contains the root side of the original polygon inherits the root, and the side that $\alpha$ lands on becomes the new root for the other subpolygon.

(3) If $\alpha$ ends on the root side of $P$, then both subpolygons inherit the original root side as their root. See Example 6.

Under this construction, dissection preserves the polygon weight of the. If $\alpha$ is a trivial arrow, then one of the resulting subpolygons is the trivial subpolygon, $1 \in V_{0}(R)=\mathbb{Q}$ whereas the other subpolygon is the original polygon. For this reason, for most of the discussion in this paper, we ignore the presence of trivial arrows.

Definition 5. A dissection $d$ of $P$ is a set of non-intersecting arrows of $P$. Denote by $D(P)$ the set of dissections of the polygon $P$, including the trivial dissection (no arrows). The cardinality of a dissection, $|d|$ is the number of non-trivial arrows in $d$.

Example 6. For example, consider $P=123456$, and dissection $d=\{\alpha$, $\beta, \gamma\}$ as drawn. Contracting along the arrows in $d$ gives the a set of four polygons.

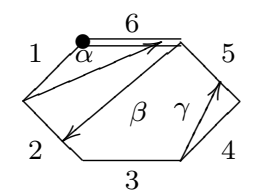

The arrow $\beta$ is an arrow not ending on the root side of $P$. Contracting along $\beta$ gives the pair of polygons

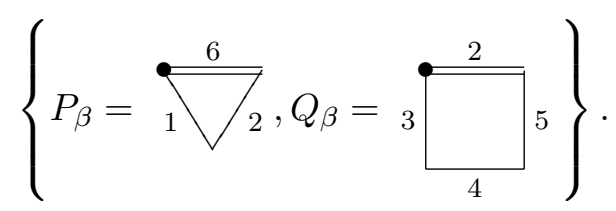

One can think of $\alpha$ now as an arrow in $P_{\beta}=126$. It ends on the root side of $P$. Contracting along both arrow $\alpha$ and $\beta$ gives the three polygons

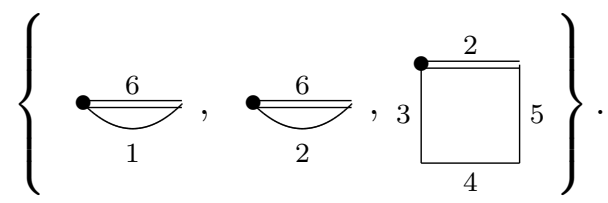


Finally, consider $\gamma$ as an arrow in $Q_{\beta}=3452$. It does not end on the root side of either $Q_{\beta}$ or $P$. Contracting all arrows in $d$ gives

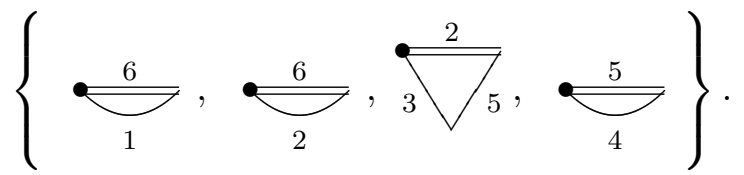

The order of contracting arrows in $d$ does not affect the set of polygons associated to it.

The polygons $P_{\alpha}$ and $Q_{\alpha}$ above are called the polygons associated to the dissecting arrow $\alpha$. If $d \in D(P)$ is a dissection with $i$ arrows, there is a set of $i+1$ subpolygons, $\left\{P_{0}, \ldots P_{i}\right\}$ associated to the dissection $d$, formed by contracting the arrows in $d$. Since dissection preserves weight, if each $P_{j} \in V_{n_{j}}(R)$, and $P \in V_{n}(R)$, then $\sum_{j=0}^{i} n_{j}=n$. Two polygons $P_{i}$ and $P_{j}$ associated to a dissection are adjacent if regions they correspond to share a dissecting arrow as a boundary.

When discussing polygons associated to dissections, it is useful to label the regions associated to dissection consisting of a single arrow. For $\alpha=d \in$ $D(P)$, the subpolygons associated to $\alpha$ are sometimes referred to as the root polygon, $P_{\alpha}^{\bullet}$, which is the subpolygon that contains the root side and first vertex of $P$, and the cut off polygon, $P_{\alpha}^{\sqcup}$, which is the other subpolygon. At other times, it is convenient to consider whether the subpolygon lies to the left or the right of the arrow, as determined by the orientation of the arrow. In this case, the left polygon is indicated $P_{\alpha}^{l}$ and the right polygon is indicated $P_{\alpha}^{r}$. Note that if $\alpha$ is a forwards arrow, $P_{\alpha}^{l}=P_{\alpha}^{\bullet}$. If it is a backwards arrow, $P_{\alpha}^{l}=P_{\alpha}^{\sqcup}$. In Example 6, since $\beta$ is a backwards arrow,

$$
P_{\beta}^{l}=P_{\beta}^{\sqcup}={ }_{3}^{\underbrace{2}_{4}}, \quad P_{\beta}^{r}=P_{\beta}^{\bullet-}=1 \underbrace{2}_{2} .
$$

Definition 7. Let $\mathcal{P}_{\bullet}^{(\star)}(R)$ be the exterior product algebra of $V_{\bullet}(R)$. It is bigraded, the subscript $\bullet$ corresponds to the weight, or Adams grading, of the vector space $V_{\bullet}(R)$, and the superscript $(\star)$ corresponds to the exterior product grading, also referred to as the degree.

The algebra $\mathcal{P}_{\bullet}^{(\star)}(R)$ can be endowed with a degree 1 differential operator to form a DGA $\left(\mathcal{P}_{\bullet}^{(\star)}(R), \partial\right)$. There are several such operators on this algebra, 
which I discuss in Section 1.4. I consider the bar constructions associated to each DGAs, $B_{\partial}\left(\mathcal{P}_{\bullet}^{(\star)}(R)\right)$.

Definition 8. Let $(\mathcal{A}, \partial)$ be a DGA with $\mathcal{A}$ a connected graded exterior product algebra, and $\partial$ a degree 1 differential operator. The bar construction $B_{\partial}(\mathcal{A})$ associated to $\left(\mathcal{A}_{\bullet}, \partial\right)$ is the reduced tensor algebra $\bar{T}\left(\mathcal{A}_{\bullet}\right)=\oplus_{i=0}^{\infty} \mathcal{A}_{\geq 1}^{\mid i}$, commutative under the shuffle product, $\amalg$, with tensor symbol denoted by |. The bicomplex structure of $B_{\partial}(\mathcal{A})$ is given by the differential operators $D_{1}$ and $D_{2}$.

The coproduct on $B_{\partial}(\mathcal{A})$ is induced from the deconcatenation coproduct on $\bar{T}(\mathcal{A})$

$$
\Delta\left[a_{1}|\cdots| a_{n}\right]=\sum_{i=0}^{n}\left[a_{1}|\cdots| a_{i}\right] \otimes\left[a_{i+1}|\cdots| a_{n}\right]
$$

It is compatible with the shuffle product on $T(\mathcal{A})$.

In this paper, I consider $\mathcal{A}_{\bullet}=\mathcal{P}_{\bullet}^{(\star)}(R)$. Given a differential operator $\partial$, the bar construction $B_{\partial}\left(\mathcal{P}_{\bullet}^{(\star)}(R)\right)$ is generated by terms of the form $\left[a_{1}|\cdots| a_{n}\right]$ where each $a_{i} \in \mathcal{P}_{\bullet}^{\left(k_{i}\right)}(R)$ is homogeneous in the exterior product grading of degree $k_{i}$.

(1) Define $D_{1}: \mathcal{P}_{\bullet}^{(\star)}(R)^{\mid n} \rightarrow \mathcal{P}_{\bullet}^{(\star)}(R)^{\mid n-1}$ to be the operator defined

$$
D_{1}\left(\left[a_{1}|\cdots| a_{n}\right]\right)=\sum_{i=1}^{n-1}-(-1)^{\sum_{j \leq i}\left(\operatorname{deg} a_{j}-1\right)}\left[a_{1}|\cdots| a_{i} \wedge a_{i+1}|\cdots| a_{n}\right] .
$$

(2) Define $D_{2}: \mathcal{P}_{\bullet}^{(\star)}(R)^{\mid n} \rightarrow \mathcal{P}_{\bullet}^{(\star)}(R)^{\mid n}$ to be the operator defined

$$
D_{2}\left(\left[a_{1}|\cdots| a_{n}\right]\right)=\sum_{j=1}^{n}(-1)^{\sum_{k<j}\left(\operatorname{deg} a_{k}-1\right)}\left[a_{1}|\cdots| \partial a_{j} \mid \cdots a_{n}\right] .
$$

Since $D_{1}$ does not involve the differential defining the DGA, this differential is the same for all $B_{\partial}\left(\mathcal{P}_{\bullet}^{(\star)}(R)\right)$. If $\partial$ and $\partial^{\prime}$ are different differential operators on $\mathcal{P}_{\bullet}^{(\star)}(R)$, the differential $D_{2}$ is different on $B_{\partial}\left(\mathcal{P}_{\bullet}^{(\star)}(R)\right)$ and $B_{\partial^{\prime}}\left(\mathcal{P}_{\bullet}^{(\star)}(R)\right)$.

Remark 9. The bar construction defined in this paper is different than the one defined in [7], specifically they differ by the overall sign of $D_{1}$. The objects in this paper have different weights than those considered in [12], 
Chapter 2, Section 2.2, otherwise, the construction in this paper and Loday and Valette agrees. It is worth noting that this bar construction also differs from that of Bloch and Kriz [1], where the shuffle product and coproduct have a very different sign convention.

\subsection{Multi-rooted trees}

In this paper, I define several Hopf algebras associated to the vector space of $R$-deco polygons $V_{\bullet}(R)$. These are defined by introducing dual tree structures to polygons and their dissections. In this subsection, I define these trees.

Definition 10. A tree is a finite contractible graph with oriented edges. Vertexes with all edges flowing away from them are called roots. Vertexes with all edges flowing into it are called leaves. A tree may have many roots, in which case is called a multi-rooted tree. If a tree has a single vertex, that vertex is both a root and a leaf.

Unlike for single-rooted trees, leaves on multi-rooted trees can have multiple edges coming into them.

Remark 11. In this paper, root vertexes are marked by a circle. I do not explicitly indicate the orientation of the edges, and leave it to be assumed from the pictures. Generally, root vertexes are drawn at the top of the tree, while the edges flow down.

Let $\mathcal{T} \bullet(R)$ be the augmented bialgebra over $\mathbb{Q}$ of multi-rooted non-planar trees with vertexes decorated by $R$-deco polygons. As with trees, a multirooted tree $T \in \mathcal{T}^{\bullet}(R)$ induces a partial order on its vertexes. A path in $T$ from the vertex $v_{1}$ in $T$ to $v_{2}$ in $T$, is a linear subtree with $v_{1}$ as a root and $v_{2}$ as leaf vertex, with orientation inherited from $T$. If $v_{1}$ and $v_{2}$ are two vertexes of a tree $T$,

$$
v_{1} \prec v_{2} \text { in } T \Longleftrightarrow \exists \text { a path in } T \text { from } v_{1} \text { to } v_{2} .
$$

A linear order of $T$ is a total ordering of the vertexes of $T$ that respects the partial order.

The algebra structure of $\mathcal{T}^{\bullet}(R)$ is given as follows. It is graded by number of vertexes in the tree

$$
\left.\mathcal{T}^{\bullet}(R)=\bigoplus_{n=0}^{\infty} \mathcal{T}^{n}(R)=\mathbb{Q}\langle T| T \text { has } n \text { vertexes }\right\rangle ; \quad \mathcal{T}^{0}(R)=\mathbb{Q} .
$$


The unit is the empty tree,

$$
\mathbb{I}_{\mathcal{T} \cdot(R)}=T_{\emptyset}
$$

The sum of two trees $T_{1}$ and $T_{2}$ is formal.

The algebra $\mathcal{T}^{\bullet}(R)$ is a commutative algebra with the product of trees being the disjoint union of trees, or a forest.

Definition 12. For a tree $T \in \mathcal{T}^{\bullet}(R)$, let $c$ be a non-empty subset of edges of $T$, and $\left\{t_{1}, \ldots t_{k}\right\}$ be the set of trees formed by removing the edges in $c$. The subset $c$ is a proper admissible cut of $T$ if, for any individual $t_{i}$, the edges of $c$ that have endpoints in $t_{i}$ either all flow into $t_{i}$ or all flow from $t_{i}$.

Example 13. For example for the tree $T={ }_{B}{ }^{A} \bigodot_{C} D$ the set $\{\alpha, \gamma\}$ is not a proper admissible cut, but the set $\{\alpha, \beta\}$ is.

Let $c$ be a proper admissible cut of $\mathcal{T}^{\bullet}(R)$, and $\left\{t_{1}, \ldots, t_{k}\right\}$ the set of subtrees of $T$ formed by removing the edges in $c$ from $T$. The definition of a proper admissible cut partitions the trees $\left\{t_{1}, \ldots, t_{k}\right\}$ by whether they are connected to the edges in $c$ by terminal vertexes, or initial vertexes. This partitions the set of subtrees in two, the set $\left\{t_{l_{1}}, \ldots, t_{l_{n}}\right\}$ of subtrees which the elements of $c$ share at most a terminal vertex in $t_{l_{i}}$, and the set $\left\{t_{r_{1}}, \ldots, t_{r_{m}}\right\}$ of subtrees which the elements of $c$ share at most an initial vertex in $t_{r_{i}}$.

Definition 14. - The leaf forest of a proper admissible cut is

$$
L(c)=\prod_{i=1}^{n} t_{l_{i}}
$$

- The root forest is

$$
R(c)=\prod_{i=1}^{m} t_{r_{i}}
$$

In the above example, for $c=\{\alpha, \beta\}$, the pruned forest is

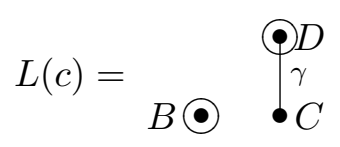


and the root forest is

$$
R(c)=\bullet A
$$

In addition to proper admissible cuts, one considers two other cuts. The empty cut is defined such that $L_{\text {empty }}(T)=1$ and $R_{\text {empty }}(T)=T$. The full cut is defined such that $L_{\text {full }}(T)=T$ and $R_{\text {full }}(T)=1$. The set of admissible cuts consists of proper admissible cuts, the empty cut and the full cut.

Definition 15. The coproduct on $\mathcal{T}^{\bullet}(R)$ is defined

$$
\Delta(T)=\sum_{c \text { admis. }} R(c) \otimes L(c) .
$$

I denote the contribution of the admissible cut $c$ to the coproduct as

$$
\Delta_{c}(T)=R(c) \otimes L(c)
$$

In this notation $\Delta(T)=\sum_{c \text { admis. }} \Delta_{c}(T)$.

Recall that in a coassociative bialgebra $\mathcal{T}^{\bullet}(R)$, for every $T \in \mathcal{T}^{\bullet}(R)$,

$$
(\Delta \otimes \mathbb{I}) \Delta(T)=(\mathbb{I} \otimes \Delta) \Delta(T) .
$$

Lemma 16. The algebra $\mathcal{T}^{\bullet}(R)$ is a coassociative Hopf algebra.

Proof. Since $\mathcal{T}^{\bullet}(R)$ is connected and graded, if it is a bialgebra, it is a Hopf algebra.

First I show that $\mathcal{T}^{\bullet}(R)$ is a bialgebra. The coproduct defined in (1.3) is compatible with multiplication on $\mathcal{T}^{\bullet}(R)$ :

$$
\Delta(T S)=\Delta(T) \Delta(S)
$$

for $S, T \in \mathcal{T}^{\bullet}(R)$. Let $L_{T}, L_{S}$ be the pruned forests of $T$ and $S$, and $R_{T}$ and $R_{S}$ the root forests of $T$ and $S$. Then

$$
\Delta(T) \Delta(S)=\sum_{d \text { admis. of } T c \text { admis. of } S} R_{S}(c) R_{T}(d) \otimes L_{S}(c) L_{T}(d) .
$$

Since the product of trees is the disjoint union, an admissible cut of $T S$ is an element of the form $d \cup c$, where $d$ is an admissible cut of $T$, and $c$ is an 
admissible cut of $S$. Therefore,

$$
\Delta(T S)=\sum_{d \cup c \text { admis. of } T S} R_{S}(c) R_{T}(d) \otimes L_{S}(c) L_{T}(d)=\Delta(T) \Delta(S) .
$$

To see coassociativity, consider $c$, an admissible cut of $T$. Write

$$
\Delta_{c}(T)=R(c) \otimes L(c)
$$

Let $c_{r}$ be an admissible cut of the forest $R(c)$. Then

$$
\left(\Delta_{c_{r}} \otimes \mathbb{I}\right) \Delta_{c}(T)=R_{c_{r}}\left(R_{c}(T)\right) \otimes L_{c_{r}}\left(R_{c}(T)\right) \otimes L_{c}(T)
$$

Since the trees in the forest $R(c)$ are subtrees of $T, c_{r}$ is also an admissible cut of $T$. The edges in $c$ are an admissible cut of the forest formed by the product $R_{c_{r}}(T) \cdot L_{c_{r}}(T)$. Write $c=c_{1} \cup c_{2}$, with $c_{1}$ an admissible cut of $R_{c_{r}}(T)$ and $c_{2}$ and admissible cut of $L_{c_{r}}(T)$. Then $c^{\prime}=c_{r} \cup c_{1}$ is an admissible cut of $T$. The components of $R_{c^{\prime}}(T)=R_{c_{1}}\left(R_{c_{r}}(T)\right)$ are attached to the source vertexes of the edges in $c^{\prime}$ while the edges in $L_{c_{1}}\left(R_{c_{r}}(T)\right)$ are attached only to the terminal vertexes of the edges in $c_{1}$. Furthermore, note that by construction,

$$
R_{c^{\prime}}(T)=R_{c_{1}}\left(R_{c_{r}}(T)\right)=R_{c_{r}}\left(R_{c}(T)\right)
$$

Since $L_{c_{1}}\left(R_{c_{r}}(T)\right)$ is part of the forest $L_{c^{\prime}}(T)$,

$$
L_{c}(T)=L_{c_{1}}\left(R_{c_{r}}(T)\right) \cdot L_{c_{2}}(T)=L_{c_{2}}\left(L_{c^{\prime}}(T)\right)
$$

Since $c_{2}$ is an admissible cut of $L_{c_{r}}(T)$,

$$
L_{c_{r}}\left(R_{c}(T)\right)=R_{c_{2}}\left(L_{c^{\prime}}(T)\right)
$$

Combining Equations (1.5) (1.6) (1.7) and (1.8) gives

$$
R_{c^{\prime}}(T) \otimes R_{c_{2}}\left(L_{c^{\prime}}(T)\right) \otimes L_{c_{2}}\left(L_{c^{\prime}}(T)\right)=\left(\mathbb{I} \otimes \Delta_{c_{2}}\right) \Delta_{c^{\prime}}(T) .
$$

Remark 17. Note that if $T \in \mathcal{T}^{\bullet}(R)$ is a single-rooted tree, the coproduct defined above matches the coproduct and definition of admissible cut in [4]. For a single-rooted tree, $R(c)$ is always a tree. If $T$ is multi-rooted, $R(c)$ may be a forest.

Definition 18. Let $W(R)$ be the algebra of non-commutative words on $R$-deco polygons. 
The algebras $W(R)$ and $\bar{T}\left(V_{\bullet}(R)\right)$ are isomorphic as commutative Hopf algebras. There is a commutative product given by the shuffle product and a coproduct given by deconcatenation. This is the same as given in the bar construction in (1.2). If $w=w_{1}, \ldots, w_{n} \in W(R)$, with the $w_{i}$ non-trivial $R$-deco polygons,

$$
\Delta w_{1} \cdots w_{n}=\sum_{i=0}^{n}\left(w_{1} \otimes \cdots \otimes w_{i}\right) \otimes\left(w_{i+1} \otimes \cdots \otimes w_{n}\right) .
$$

There is a natural identification

$$
(W(R), \amalg, \Delta) \simeq\left(\bar{T}\left(V_{\bullet}(R)\right), \amalg, \Delta\right) \simeq\left(\bar{T}\left(\mathcal{P}_{\bullet}^{(1)}(R)\right), \amalg, \Delta\right) .
$$

There is an algebra homomorphism from the algebra of trees, $\mathcal{T}^{\bullet}(R)$, to the algebra of words, $W(R)$, which identifies the partial order represented by $T$ with a sum of words in $W(R)$. I first need to define linearizations of trees.

Definition 19. For $T \in \mathcal{T}^{n}(R)$, a partial order preserving a linearization of $T$ is a word on $R$-deco polygons

$$
\lambda(T)=\lambda_{1} \otimes \lambda_{2} \otimes \cdots \otimes \lambda_{n} \in W(R)
$$

where each $\lambda_{i}$ is an $R$ deco polygon labeling a vertex of $T$. If $\lambda_{i} \prec \lambda_{j}$ as vertexes in $T$, then $i<j$.

Let $\operatorname{Lin}(T)$ be the set of partial order preserving linearizations of trees. For any $\lambda \in \operatorname{Lin}(T)$, the polygon $\lambda_{1}$ is always the label of a root of $T$ and $\lambda_{n}$ is always the label of a leaf of $T$. A forest in $\mathcal{T}^{\bullet}(R)$ also represents a partial order on its vertexes. The linearization of trees extends naturally to forests.

In this paper, the partial order of $T$ is viewed as the sum of its partial order preserving linearizations. I define a map from trees to words by mapping each tree to the sum of its linearizations:

$$
\begin{aligned}
\Lambda: \mathcal{T}^{\bullet}(R) & \rightarrow W(R) \\
T & \mapsto \sum_{\lambda \in \operatorname{Lin}(T)} \lambda(T) \\
T^{\prime} \cdot T & \mapsto \sum_{\lambda^{\prime} \in \operatorname{Lin}\left(T^{\prime}\right)} \lambda^{\prime}\left(T^{\prime}\right) \amalg \sum_{\lambda \in \operatorname{Lin}(T)} \lambda(T)=\Lambda(T) \amalg \Lambda\left(T^{\prime}\right),
\end{aligned}
$$

where $\amalg$ is the shuffle product on $W(R)$. 
Example 20. Let $T$ be the tree,

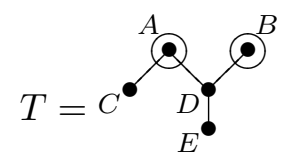

It has two root vertexes $A$ and $B$. Then

$$
\lambda(T)=A \otimes B \otimes C \otimes D \otimes E \quad \text { and } \quad \lambda^{\prime}(\mathrm{T})=\mathrm{B} \otimes \mathrm{A} \otimes \mathrm{D} \otimes \mathrm{E} \otimes \mathrm{C}
$$

are two partial order preserving linearizations of $T$. The sum of all partial ordered preserving linearizations is

$$
\Lambda(T)=(A \amalg B) \otimes(C \amalg(D \otimes E))+A \otimes C \otimes B \otimes D \otimes E .
$$

Theorem 21. The map $\Lambda: \mathcal{T}^{\bullet}(R) \rightarrow W(R)$ is a bialgebra homomorphism.

Proof. The algebra homomorphism comes from the construction of the map $\Lambda$. The coalgebra homomorphism is shown here.

For $T \in \mathcal{T}^{n}(R)$, the coproduct on $T$ is

$$
\Delta(T)=\sum_{c \text { admis. }} R(c) \otimes L(c)
$$

and the coproduct on the image, $\Lambda(T)$, is

$$
\Delta \Lambda(T)=\sum_{i=0}^{n} \sum_{\lambda \in \operatorname{Lin}(T)}\left(\lambda_{1} \otimes \ldots \otimes \lambda_{i}\right) \otimes\left(\lambda_{i+1} \otimes \ldots \otimes \lambda_{n}\right)
$$

Any decomposition of a partial order preserving linearization $\lambda(T),\left[\lambda_{1} \otimes\right.$ $\left.\cdots \otimes \lambda_{i}\right]$ and $\left[\lambda_{i+1} \otimes \cdots \otimes \lambda_{n}\right]$, can be written as a tensor product of partial order preserving linearization of forests of the form $\rho(R)$ and $\eta(L)$ with $R$ and $L$ sub-forests of $T$ defined by the vertex sets $\left\{\lambda_{1} \cdots \lambda_{i}\right\}$ and $\left\{\lambda_{i+1} \cdots \lambda_{n}\right\}$, respectively. The set of edges of $T$ that connect the vertexes $\lambda_{j}$ to $\lambda_{k}$ for $j \leq i$ and $k>i$ define an admissible cut of $T$.

For each admissible cut $c$, the trees in the forests $L(c)$ and $R(c)$ are subtrees of $T$. Let $\eta_{c} \in \operatorname{Lin}(L(c))$ and $\rho_{c} \in \operatorname{Lin}(R(c))$ be partial order preserving 
linearizations. Then

$$
(\Lambda \otimes \Lambda) \circ \Delta(T)=\sum_{c \text { admis. }} \sum_{\substack{\left(\eta_{c}, \rho_{c}\right) \in \\ \operatorname{Lin}(R(c)) \times \operatorname{Lin}(L(c))}} \rho_{c} \otimes \eta_{c},
$$

where the interior sum is taken over all partial order preserving linearizations of $R(c)$ and $L(c)$. By definition of admissible cut, each pair of partial order preserving linearizations $\rho_{c} \otimes \eta_{c}$, corresponds to a decomposition of a partial order preserving linearizations $\lambda$ of $T,\left[\lambda_{1} \otimes \cdots \otimes \lambda_{i}\right] \otimes\left[\lambda_{i+1} \otimes \cdots \otimes \lambda_{n}\right]$ where the vertexes of $R(c)$ precede the vertexes of $L(c)$.

Remark 22. Words in $W(R)$ represent a partial order on its letters. For $u$ and $v$ letters in $W=\sum_{i} W_{i} \in W(R), u \prec v$ if $u=x_{i_{j}}$ and $v=x_{i_{k}}$ in $W_{i}$ with $i_{j} \leq i_{k}$. Similarly, $v \prec u$ if $u=x_{i_{j}}$ and $v=x_{i_{k}}$ in $W_{i}$ with $i_{j} \geq i_{k}$. Under this definition, the map $\Lambda$ is an order preserving Hopf algebra homomorphism.

To complete the analysis in this paper, I need to introduce a method of inserting letters into words.

Definition 23. Define two insertion products on $W(R)$

$$
\begin{aligned}
& u \star_{\prec v}: W(R) \rightarrow W(R) \\
& x_{1} \otimes \cdots \otimes x_{n} \rightarrow \begin{cases}\sum_{i<k} x_{1} \otimes \cdots u \otimes x_{i} \cdots \otimes x_{n}, & \text { if } v=x_{k} \\
0, & v \notin\left\{x_{1}, \ldots, x_{k}\right\}\end{cases}
\end{aligned}
$$

and

$$
\begin{aligned}
& u \star_{\succ v}: W(R) \rightarrow W(R) \\
& x_{1} \otimes \cdots \otimes x_{n} \rightarrow\left\{\begin{array}{ll}
\sum_{i>k} x_{1} \otimes \cdots x_{i} \otimes u \cdots \otimes x_{n}, & \text { if } v=x_{k} \\
0 & v \notin\left\{x_{1}, \ldots, x_{k}\right\}
\end{array} .\right.
\end{aligned}
$$

If $w=1$ then

$$
u \star_{\prec 1} 1=u \star_{\succ 1} 1=u .
$$

To see this as a product, generalize the insertion of a letter to the intertwining of a word with another. 
Definition 24. Let $w$ and $w^{\prime}$ be two words. Define a set of words $\left\{W_{i} \mid i \in I\right\}$ such that $w \amalg w^{\prime}=\sum_{i \in I} W_{i}$,

$$
\left(w, w^{\prime}\right) \rightarrow \begin{cases}\sum_{\substack{i \in I \text { s.t. } \\ u \prec v \\ 0,}}^{\star_{u \prec v}: W(R) \otimes W(R) \rightarrow W(R)}, \quad \text { if } u \text { and } v \text { letters of } w \text { and } w^{\prime} \text { resp. } \\ \text { if } u \text { or } v \text { not letters of } w \text { and } w^{\prime} \text { resp. }\end{cases}
$$

Similarly,

$$
\left(w, w^{\prime}\right) \rightarrow \begin{cases}\sum_{\substack{i \in I \text { s.t. } \\ u \succ v \\ 0,}}^{\star_{u \succ v}: W(R) \otimes W(R) \rightarrow W(R)} W_{i}, & \text { if } u \text { and } v \text { letters of } w \text { and } w^{\prime} \text { resp. } \\ \text { if } u \text { or } v \text { not letters of } w \text { and } w^{\prime} \text { resp. }\end{cases}
$$

In the product $w \star_{u \prec v} w^{\prime}$, the letter $u \prec v$, while $v \succ u$ in $w \star_{u \succ v} w^{\prime}$. In this notation, $u \star_{\prec v} w:=u \star_{u \prec v} w$ and $u \star_{\succ v} w:=u \star_{u \succ v} w$.

For further analysis, I extend this product for shuffles of words. For $v$ a letter of the word $w$ and $v^{\prime}$ a letter of the word $w^{\prime}$, define

$$
\left(u \star_{\prec\left\{v, v^{\prime}\right\}} w \amalg w^{\prime}\right):=\left(u \star_{\prec v} w\right) \star_{u \prec v^{\prime}} w^{\prime},
$$

and

$$
\left(u \star_{\succ\left\{v, v^{\prime}\right\}} w \amalg w^{\prime}\right):=(u \star \succ v w) \star_{u \succ v^{\prime}} w^{\prime} .
$$

These operators can be lifted to grafting operators on trees. If $w=\Lambda(T)$, and $w^{\prime}=\Lambda\left(T^{\prime}\right)$, for $T$, and $T^{\prime}$ in $\mathcal{T}^{\bullet}(R)$,

$$
\left(u \star \prec\left\{v, v^{\prime}\right\} w \amalg w^{\prime}\right)=\Lambda(S)
$$

where $S$ is the multi-rooted tree formed by connecting the vertex labeled $v$ in $T$ and the vertex labeled $v^{\prime}$ in $T^{\prime}$ to a new root vertex labeled $u$. The other insertion operator corresponds to connecting the two marked vertexes to a new leaf, with the label $u$.

The coproduct on the images of these insertion operators behaves as follows. 
Lemma 25. Write $w=x_{1} \otimes \cdots \otimes x_{n}$. The coproduct

$$
\begin{aligned}
\Delta\left(u \star \prec x_{k} w\right)= & \sum_{a=0}^{k-1}\left(x_{1} \otimes \cdots \otimes x_{a}\right) \otimes u \prec_{\prec x_{k}}\left(x_{a+1} \otimes \cdots \otimes x_{n}\right) \\
& +\left(x_{1} \otimes \cdots \otimes x_{k-1}\right) \amalg u \otimes\left(x_{k} \otimes \cdots \otimes x_{n}\right) \\
& +\sum_{a=k}^{n} u \star \prec x_{k}\left(x_{1} \otimes \cdots \otimes x_{a}\right) \otimes\left(x_{a+1} \otimes \cdots \otimes x_{n}\right) .
\end{aligned}
$$

Similarly, the coproduct The coproduct

$$
\begin{aligned}
\Delta\left(u \star_{\succ x_{k}} w\right)= & \sum_{a=0}^{k-1}\left(x_{1} \otimes \cdots \otimes x_{a}\right) \otimes u \star_{\succ x_{k}}\left(x_{a+1} \otimes \cdots \otimes x_{n}\right) \\
& +\sum_{a=k}^{n} u \star_{\succ x_{k}}\left(x_{1} \otimes \cdots \otimes x_{a}\right) \otimes\left(x_{a+1} \otimes \cdots \otimes x_{n}\right) \\
& +\left(x_{1} \otimes \cdots \otimes x_{k}\right) \otimes u \amalg\left(x_{k+1} \otimes \cdots \otimes x_{n}\right) .
\end{aligned}
$$

Proof. The proof is straight forward from the definition of coproduct on all words in the sum $u \amalg w$, such that the letter $u$ appears to the left of $x_{k}$, in the case of $\star \prec x_{k}$, or to the right of $x_{k}$, in the case of $\star \succ x_{k}$.

\subsection{Hopf algebras of trees associated to dissected polygons}

To continue to generalizing the construction in [7], I associate a family of (multi-rooted) tree structures to each dissection of a polygon. In Section 1.4, I define a family of bar complexes on $\mathcal{P}_{\bullet}^{(\star)}(R)$ and associate to each polygon a bar element in each bar complex.

The rest of this paper is concerned with subalgebras $\mathfrak{T}_{\phi} \subset \mathcal{T}^{\bullet}(R)$ generated by sets corresponding to polygons and their dissections.

Definition 26. A dual tree algebra $\mathfrak{T}_{\phi} \subset \mathcal{T}^{\bullet}(R)$ is generated by a dual tree generating set, which assigns to each polygon dissection pair $(P, d)$ an element of $\mathcal{T}^{\bullet}(R), T_{\phi, d}(P)$,

$$
\phi=\left\{T_{\phi, d}(P) \mid P R-\text { deco polygon; } \quad d \in D(P)\right\} .
$$

In this paper, the generators, $T_{\phi, d}(P) \in \mathcal{T}^{\bullet}(R)$ are trees with an overall sign. 
Definition 27. I write the overall sign associated to a $\operatorname{tree} \operatorname{sign}\left(T_{\phi, d}(P)\right)$. For $d \in D(P)$, sometimes I write this $\operatorname{sign}_{\phi}(P, d)$. When the polygon is clear, I write $\operatorname{sign}_{\phi}(d)$.

I am particularly interested in the cases when $\mathfrak{T}_{\phi}$ has a Hopf algebraic structure.

Definition 28. The dual tree algebra $\mathfrak{T}_{\phi}$ is a dissection compatible Hopf algebra if

(1) The dual tree algebra $\mathfrak{T}_{\phi}$, generated by the set $\phi$, is a sub-Hopf algebra of $\mathcal{T}^{\bullet}(R)$.

(2) The edges of each tree $T_{\phi, d}(P)$ correspond to non-trivial arrows in $d$.

(3) Let $d^{\prime}$ be a sub-dissection of $d, d^{\prime} \subset d \in D(P)$, corresponding to the subtree $T$ of $T_{\phi, d}(P)$. There exists an $R$-deco polygon $Q$ such that $T$ and $T_{\phi, d^{\prime}}(Q)$, with $d^{\prime} \in D(Q)$, agree up to a sign. In fact

$$
\operatorname{sign}\left(T_{\phi, d^{\prime}}(Q)\right) T=T_{\phi, d^{\prime}}(Q) .
$$

(4) Consider $d^{\prime} \subset d \in D(P)$ as above. The generator $T_{\phi, d \backslash d^{\prime}}(P)$ is formed by replacing the subtree $T$ in $T_{\phi, d}(P)$ with a single vertex labeled $Q$.

(5) For each subdissection $d^{\prime} \subset d$, with corresponding generator $T_{\phi, d^{\prime}}(Q)$,

$$
\operatorname{sign}\left(T_{\phi, d}(P)\right)=\operatorname{sign}\left(T_{\phi, d^{\prime}}(Q)\right) \operatorname{sign}\left(T_{\phi, d \backslash d^{\prime}}(P)\right) .
$$

The third condition of Definition 28 ensures that a dissection compatible Hopf algebra is coassociative. Note that there is no requirement that the labels of the vertexes of $T_{\phi, d}(P)$ correspond to the subpolygons associated to the dissection. However, conditions 3 and 4 impose strong conditions on the vertex labels of the generators of dissection compatible Hopf algebras. In most of the examples I consider in this paper, the vertexes of the generators are labeled by the the subpolygons associated to the relevant dissection. Below I give some examples of some generators of dissection compatible Hopf algebras.

Example 29. Consider the following pair of polygon and dissection:

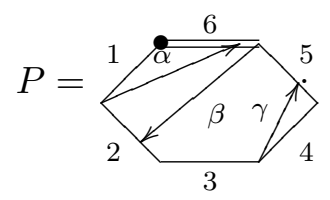


Four possible elements of dual tree generating sets are
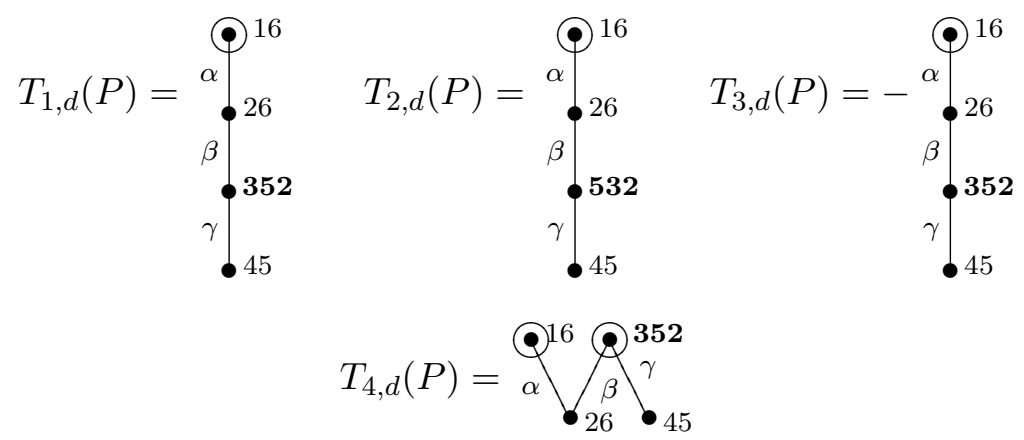

For a fixed $P$ and $d \in D(P)$, the generators $T_{1, d}(P)$ and $T_{3, d}(P)$ differ only by an overall sign. The only multi-rooted tree in this example is $T_{4, d}(P)$. The vertexes in $T_{2, d}(P)$ do not correspond to the set of subpolygons associated to the dissections $d$ of $P$. Instead of a vertex labeled with the polygon 352 , there is a polygon labeled 532 .

Next I give examples of four dual tree generating sets $\phi_{i}$, with $i \in$ $\{1, \ldots, 4\}$ such that $\mathfrak{T}_{\phi_{i}}$ is a dissection compatible Hopf algebra. The generators $T_{i, d}$ in Example 29 correspond to elements in $\phi_{i}$. Before defining these Hopf algebras and the construction of the corresponding generators, I establish some notation.

Definition 30. Let $\tau$ be a map that reverses the orientation of a polygon.

Specifically, for $P=r_{1} \cdots r_{n+1}$, with $r_{i} \in R, \tau \in D_{2(n+1)}$ and $\tau(P)=$ $r_{n} \cdots r_{1} r_{n+1}$. For example,
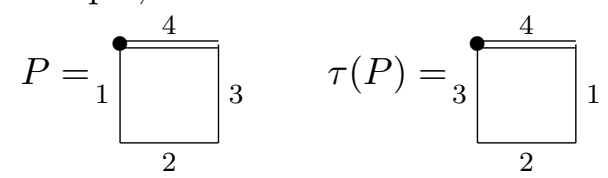

Definition 31. Define $\chi(\alpha)$ to be the weight of the cut off polygon of the arrow $\alpha$. That is, $P_{\alpha}^{\sqcup} \in \mathcal{P}_{\chi(\alpha)}^{(1)}(R)$.

Example 32. The following are four dissection compatible Hopf algebras.

$\mathfrak{T}_{\phi_{1}}$ : This is a single-rooted dual tree algebra generated by the set $\phi_{1}$. The root vertex of $T_{\phi_{1}, d}(P) \in \phi_{1}$ is labeled by the subpolygon that contains the original root side and first vertex of $P$. The edges, corresponding to arrows in $d$, are oriented to flow away from the root vertex. Since this is 
a single-rooted tree, I need only consider the final vertexes of any edge (corresponding to the dissecting arrow $\alpha$.) The initial vertex is either the final vertex of a different arrow, or the unique root, whose label has been defined. Consider the edge corresponding to the dissecting arrow $\alpha$. The final vertex is labeled by the subpolygon corresponding to the region further away from the first vertex/root side of $P$. In Example 29, $T_{1, d}(P) \in \phi_{1}$.

$\mathfrak{T}_{\phi_{2}}$ : This is a single-rooted dual tree algebra generated by the set $\phi_{2}$. The single root vertex of $T_{\phi_{2}, d}(P) \in \phi_{2}$ is labeled by the subpolygon that contains the original root side and first vertex of $P$. The edges, corresponding to arrows in $d$, are oriented to flow away from the root vertex. If $\alpha$ is a forwards arrow, then the final vertex is labeled by the subpolygon corresponding to the region further from the root. If $\alpha$ is a backwards arrow, then the final vertex is labeled by the same polygon with reversed orientation. The generator has an overall sign

$$
\operatorname{sign}\left(T_{\phi_{2}, d}(P)\right)=(-1)^{\sum_{\alpha \in d \text { backwards }} \chi(\alpha)} .
$$

In Example 29, $T_{2, d}(P) \in \phi_{2}$.

$\mathfrak{T}_{\phi_{3}}$ : This is a single-rooted dual trees algebra generated by the set $\phi_{3}$. The single root vertex of $T_{\phi_{3}, d}(P) \in \phi_{3}$ is labeled by the subpolygon that contains the original root side and first vertex of $P$. The edges, corresponding to arrows in $d$, are oriented to flow away from the root vertex. The final vertex is labeled by the subpolygon corresponding to the region further away from the first vertex/root side of $P$. The generator has an overall sign

$$
\operatorname{sign}\left(T_{\phi_{3}, d}(P)\right)=(-1)^{\# \text { backwards arrows in } d} .
$$

In Example 29, $T_{3, d}(P) \in \phi_{3}$.

$\mathfrak{T}_{\phi_{4}}$ : This is a multi-rooted dual tree algebra generated by the set $\phi_{4}$, with generators $T_{\phi_{4}, d}(P) \in \phi_{4}$. The edges, corresponding to arrows in $d$, flow from the region to the left of the arrow to the region to the right. The initial vertex of an edge is labeled with the subpolygon associated to the region to the left, and the final vertex by the subpolygon associated to the right. For the polygon pair in Example 29, $T_{4, d}(P) \in \phi_{4}$. 
In the dissection compatible sub-Hopf algebras in Example 32, write the generating set of $\mathfrak{T}_{\phi_{i}}$

$$
\phi_{i}=\left\{T_{\phi_{i}, d}(P) \mid d \in D(P) ; \quad P R-\text { deco polygon }\right\}
$$

for $i \in\{1,2,3,4$,$\} . Note that the different generating sets \phi_{1}$ and $\phi_{3}$ generate isomorphic Hopf algebras $\mathfrak{T}_{\phi_{1}} \simeq \mathfrak{T}_{\phi_{3}}$ under the relation

$$
T_{\phi_{1}, d}(P)=\operatorname{sign}_{\phi_{3}}(d)\left(T_{\phi_{3}, d}(P)\right) \text {. }
$$

Each $\mathfrak{T}_{\phi_{i}}$ satisfies conditions $2-5$ of Definition 28. To see that these are dissection compatible Hopf algebras, it remains to check that they are subHopf algebras. The Hopf algebra $\mathfrak{T}_{\phi_{2}}$ is exactly the Hopf algebra defined in [7], Section 6. It remains to check that $\mathfrak{T}_{\phi_{1}}, \mathfrak{T}_{\phi_{3}}$ and $\mathfrak{T}_{\phi_{4}}$ are Hopf algebras.

Lemma 33. The algebras $\mathfrak{T}_{\phi_{1}}, \mathfrak{T}_{\phi_{3}}$ and $\mathfrak{T}_{\phi_{4}}$ are Hopf algebras.

Proof. Since all three are graded subalgebras of $\mathcal{T} \bullet(R)$, it is sufficient to show that these are sub-bialgebras. The product structure and coproduct structure on each are inherited from $\mathcal{T}^{\bullet}(R)$. It remains to check that

$$
\Delta: \mathfrak{T}_{\phi_{i}} \rightarrow \mathfrak{T}_{\phi_{i}} \otimes \mathfrak{T}_{\phi_{i}}
$$

for $i \in\{1,3,4\}$. For this, it is sufficient to work only with the generators.

Let $\left\{P_{1}, \ldots, P_{|d|+1}\right\}$ be the vertexes labeling $T_{\phi_{4}, d}(P)$. Since the edges of $T_{\phi_{4}, d}(P)$ correspond to arrows to the dissection $d$, an admissible cut, $c$, of $T_{\phi_{4}, d}(P)$ can be thought of as a subdissection $c \subset d$. Let $\left\{Q_{1}, \ldots Q_{n}\right\}$ be the polygons associated to the dissection $c \in D(P)$, with $R(c)=\prod_{i=1}^{j} T_{i}$ and $L(c)=\prod_{i=j+1}^{n} T_{i}$. Write $d=\left(\cup_{k=1}^{n} d_{i}\right) \cup c$ with the subdissection $d_{i}$ corresponding to the edges in $T_{i}$. It remains to check that

$$
T_{i}=\operatorname{sign}_{\phi_{4}}\left(d_{i}\right) T_{\phi_{4}, d_{i}}\left(Q_{i}\right)
$$

For each $\beta \in d \backslash c$, let $P_{k}$ and $P_{j}$ be the regions of $P$ to the left and right of $\beta$, respectively. By definition, $\beta \in d_{i}$ for some $i$. Then $P_{k}$ and $P_{j}$ are also the sub-regions of $Q_{i}$ to the left and right of $\beta \in d_{i}$. Thus, $T_{d_{i}}=T_{4, d_{i}}\left(Q_{i}\right)$.

The argument is similar for $\mathfrak{T}_{\phi_{1}}$ and $\mathfrak{T}_{\phi_{3}}$. Since they are isomorphic, it is sufficient to work only with $\phi_{1}$. Let $\left\{P_{1}, \ldots, P_{|d|+1}\right\}$ be the vertex labels of $T_{\phi_{1}, d}(P) \in \phi_{1}$. Consider the admissible cut $c$ with $L(c)=\prod_{i=1}^{n-1} T_{i}$ and $R(c)=T_{n}$. Then $\left\{Q_{1}, \ldots, Q_{n}\right\}$ are the polygons associated to $c$. The dissection $d$ can be written $d=\left(\cup_{i=1}^{n} d_{i}\right) \cup c$ with $d_{i}$ corresponding to the edges 
of $T_{i}$. For each $\beta \in d \backslash c$, let $P_{i}$ be the region of $P$ on the root side of $\beta$ and $P_{j}$ on the cutoff side. Let $\beta \in d_{k}$. Then $P_{i}$ and $P_{j}$ correspond to the regions corresponding to the root and the cutoff sides of $\beta \in d_{k} \in D\left(Q_{k}\right)$. Thus $T_{i}=\operatorname{sign}_{\phi_{1}}\left(d_{i}\right) T_{\phi_{1}, d_{i}}\left(Q_{i}\right)$.

\subsection{From generating sets to differentials}

A dual tree generating set that defines a dissection compatible Hopf algebra also defines a degree one differential on $\mathcal{P}_{\bullet}^{(\star)}(R), \partial: \mathcal{P}_{\bullet}^{(i)}(R) \rightarrow \mathcal{P}_{\bullet}^{(i+1)}(R)$ satisfying $\partial \circ \partial=0$ and the Leibniz rule

$$
\partial_{\phi}(a \wedge b)=\left(\partial_{\phi} a\right) \wedge b+(-1)^{i} a \wedge \partial_{\phi}(b)
$$

where $a \in \mathcal{P}_{\bullet}^{(i)}(R)$.

Let $\phi$ be a dual tree generating set. Consider the subset of dissections $d \in D(P)$ such that $|d|=1$. Write the corresponding elements of $\phi$

$$
T_{\phi, d}(P)=\operatorname{sign}_{\phi}(d) \bullet_{\bullet P_{d}^{2}}^{P_{d}^{1}} .
$$

This structure defines a an operator on $\mathcal{P}_{\bullet}^{(\star)}(R)$.

Definition 34. Define

$$
\partial_{\phi}(P)=\sum_{d \in D(P) ;|d|=1} \operatorname{sign}_{\phi}(d) P_{d}^{1} \wedge P_{d}^{2} .
$$

Theorem 35. If $\phi$ generates a dissection compatible Hopf algebra, $\mathfrak{T}_{\phi}$, then $\partial_{\phi}$ is a degree one differential operator on $\mathcal{P}_{\bullet}^{(\star)}(R)$.

Proof. By construction,

$$
\begin{aligned}
\partial_{\phi} \circ \partial_{\phi}(P)= & \sum_{\substack{\alpha, \beta \\
\text { dis. arrow }}}\left(P_{\beta}^{1}\right)_{\alpha}^{1} \wedge\left(P_{\beta}^{1}\right)_{\alpha}^{2} \wedge P_{\beta}^{2}-P_{\beta}^{1} \wedge\left(P_{\beta}^{2}\right)_{\alpha}^{1} \wedge\left(P_{\beta}^{2}\right)_{\alpha}^{2} \\
& +\left(P_{\alpha}^{1}\right)_{\beta}^{1} \wedge\left(P_{\alpha}^{1}\right)_{\beta}^{2} \wedge P_{\alpha}^{2}-P_{\alpha}^{1} \wedge\left(P_{\alpha}^{2}\right)_{\beta}^{1} \wedge\left(P_{\alpha}^{2}\right)_{\beta}^{2} .
\end{aligned}
$$

Some of these terms are 0 . For instance, if $\beta \notin D\left(P_{\alpha}^{i}\right)$ then $D\left(P_{\alpha}^{i}\right)_{\beta}^{i}=0$. 
This can be calculated by considering the sum

$$
\left(\Delta_{\alpha} \otimes \mathbb{I}\right) \Delta_{\beta}-\left(\mathbb{I} \otimes \Delta_{\alpha}\right) \Delta_{\beta}+\left(\Delta_{\beta} \otimes \mathbb{I}\right) \Delta_{\alpha}-\left(\mathbb{I} \otimes \Delta_{\beta}\right) \Delta_{\alpha}
$$

on the level of Hopf algebras, and passing from the tensor product to the wedge product.

Ignoring the sign and the vertex labels, the generator $T_{\phi,\{\alpha, \beta\}}(P)$ is one of three possible trees
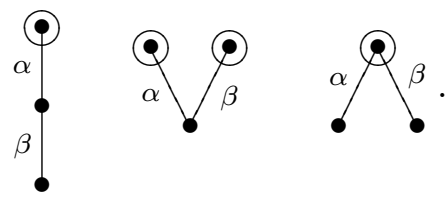

In the case of the linear tree, the first two terms of (1.9) are equal, and therefore cancel. The other two are 0. For the non-linear single-rooted tree, the sum of the first and third terms in (1.9) gives $\left(P_{\beta}^{1}\right)_{\alpha}^{1} \otimes P_{\beta}^{2} \amalg P_{\alpha}^{2}$. The other two are 0 . For the non-linear multi-rooted tree, the sum of the second and fourth terms in (1.9) gives $P_{\beta}^{1} \amalg P_{\alpha}^{2} \otimes\left(P_{\alpha}^{2}\right)_{\beta}^{2}$. The other two are 0 . In both cases, the shuffle product goes to zero as one passes to the wedge product.

Thus, $\partial_{\phi} \circ \partial_{\phi}=0$ as desired.

Since the dual tree generating sets $\phi_{i}, i \in\{1,2,3,4\}$ in Example 32 generate dissection compatible Hopf algebras $\mathfrak{T}_{\phi_{i}}$, they define degree 1 differential operators $\partial_{i}$, respectively on $\mathcal{P}_{\bullet}^{(\star)}(R)$.

Example 36. The differentials defined by the sets $\phi_{1}, \phi_{2}, \phi_{3}$ and $\phi_{4}$ are

(1) $\partial_{1}(P)=\sum_{d \in D(P),|d|=1} P_{d}^{\bullet} \wedge P_{d}^{\sqcup}$

(2) $\partial_{2}(P)=\sum_{d \text { forwards arrow }} P_{d}^{\bullet-} \wedge P_{d}^{\sqcup}+\sum_{d \text { backwards arrow }}(-1)^{\chi(d)}$ $P_{d}^{\bullet} \wedge \tau\left(P_{d}^{\sqcup}\right)$

(3) $\partial_{3}(P)=\sum_{d \text { forwards arrow }} P_{d}^{\bullet-} \wedge P_{d}^{\sqcup}-\sum_{d \text { backwards arrow }} P_{d}^{\bullet-} \wedge P_{d}^{\sqcup}$

(4) $\partial_{4}(P)=\sum_{d \in D(P),|d|=1} P_{d}^{l} \wedge P_{d}^{r}$

These differentials defined by dual tree generating sets are not all distinct. Specifically,

$$
\operatorname{sign}_{\phi_{3}}(\alpha)= \begin{cases}\operatorname{sign}_{\phi_{4}}(\alpha), & \text { if } \alpha \text { forwards } \\ -\operatorname{sign}_{\phi_{4}}(\alpha), & \text { if } \alpha \text { backwards. }\end{cases}
$$


As a result, $\partial_{3}=\partial_{4}$.

Definition 37. The difference set between two dual tree generating sets $\phi$ and $\psi$ is

$$
\begin{gathered}
\mathcal{S}=\left\{\alpha \in D(P) \| \alpha \mid=1 ; P R-\operatorname{deco} ; T_{\phi, \alpha}(P)=\operatorname{sign}_{\phi}(\alpha) \bullet_{P_{\alpha}^{2}}^{P_{\alpha}^{1}}\right. \\
\text { and } \left.T_{\psi, \alpha}(P)=-\operatorname{sign}_{\phi}(\alpha) \bullet_{P_{\alpha}^{1}}^{P_{\alpha}^{2}}\right\} .
\end{gathered}
$$

This condition on single dissections can be generalized to general trees.

Definition 38. Let $\mathfrak{T}_{\phi}$ be a dissection compatible Hopf algebra. Let $\mathcal{S}$ be the difference set between $\phi$ and another dual tree generating set $\psi$. The dual tree algebra $\mathfrak{T}_{\psi}$ is almost $\phi$ compatible if, for any dissection $d$ of any $R$ deco polygons $P, \operatorname{sign}_{\psi}(P, d)=(-1)^{|d \cap \mathcal{S}|} \operatorname{sign}_{\phi}(P, d)$, and the tree underlying the generator $T_{\psi, d}(P)$ is formed by reversing the orientation of the edges of $T_{\phi, d}(P)$ in $d \cap \mathcal{S}$.

The dissection compatible Hopf algebra, $\mathfrak{T}_{\phi}$ is trivially an almost $\phi$ compatible algebra.

Corollary 39. Let $\mathfrak{T}_{\phi}$ be a dissection compatible Hopf algebra, and $\mathfrak{T}_{\phi^{\prime}}$ an almost $\phi$ compatible algebra. Then $\partial_{\phi}=\partial_{\phi^{\prime}}$.

Proof. Let $\mathcal{S}$ be the difference set between $\phi$ and $\phi^{\prime}$. By definition,

$$
\begin{aligned}
\partial_{\phi}(P) & =\sum_{d \in D(P) ;|d|=1} \operatorname{sign}_{\phi}(d) P_{d}^{1} \wedge P_{d}^{2} \\
& =\sum_{d \notin D(P) \cap \mathcal{S}} \operatorname{sign}_{\phi}(d) P_{d}^{1} \wedge P_{d}^{2}-\sum_{d \in D(P) \cap \mathcal{S}} \operatorname{sign}_{\phi}(d) P_{d}^{2} \wedge P_{d}^{1} \\
& =\partial_{\phi^{\prime}}(P) .
\end{aligned}
$$

Since $\mathfrak{T}_{\phi^{\prime}}$ is almost $\phi$ compatible, $\partial_{\phi^{\prime}} \circ \partial_{\phi^{\prime}}=0$.

Almost compatible algebras are particularly important for the calculations in Section 2. I give an example of a such below.

Definition 40. Let $r e(P)$ be the set of non-trivial arrows ending on the root side of an $R$-deco polygon $P$ (the root ending arrows). To fix notation, 
for $P$ an $n$-gon, write $r e(P)=\left\{{ }_{2} \alpha, \ldots,{ }_{n-1} \alpha\right\}$, where ${ }_{i} \alpha$ starts at the $i$ th vertex.

Example 41. I define a dual tree algebra, $\mathfrak{T}_{\phi_{r e}}$ generated by the set of single-rooted trees $\phi_{\text {re }}$. For any dissection $d \in D(P)$, the root vertex of $T_{\phi_{\text {re }}, d}(P) \in \phi_{\text {re }}$ is labeled by the subpolygon that contains the original root side and last vertex of $P$. The edges of the generator are oriented to flow away from the root vertex. Consider the edge corresponding to the dissecting arrow $\alpha$. The initial vertex of $\alpha$ is labeled by the subpolygon corresponding to the region closer to the last vertex/root side of $P$. If $\alpha$ is a forwards arrow, then the final vertex is labeled by the subpolygon corresponding to the region further away. If $\alpha$ is a backwards arrow, then the final vertex is labeled by the same polygon with reversed orientation. The generator has an overall sign

$$
\begin{aligned}
\operatorname{sign}\left(T_{\phi_{r e}, d}(P)\right) & =(-1)^{|d \cap r e(P)|}(-1)^{\sum_{\alpha \in d \text { backwards }} \chi(\alpha)} \\
& =(-1)^{|d \cap r e(P)|} \operatorname{sign}_{\phi_{2}}(d) .
\end{aligned}
$$

The algebra $\mathfrak{T}_{\phi_{r e}}$ is almost $\phi_{2}$ compatible. The difference set between $\phi_{\text {re }}$ and $\phi_{2}$ is

$$
\mathcal{S}=\bigcup_{P R-\operatorname{deco}} \operatorname{re}(P)
$$

If $\alpha$ is a backwards arrow, then $\alpha \notin r e(P)$ by construction, and

$$
T_{\phi_{r e}, \alpha}(P)=(-1)^{\chi(\alpha)} \bigodot_{\bullet \tau\left(P_{\alpha}^{\sqcup}\right)}^{P_{\alpha}^{\bullet}} \quad ; \quad T_{\phi_{2}, \alpha}(P)=(-1)^{\chi(\alpha)} \boldsymbol{\bullet}_{\tau\left(P_{\alpha}^{\sqcup}\right)}^{P_{\alpha}^{\bullet}}
$$

Similarly, if $\alpha$ is a forwards arrow $\alpha \notin r e(P)$,

$$
T_{\phi_{r e}, \alpha}(P)=\bigodot_{\bullet P_{\alpha}^{\sqcup}}^{P_{\alpha}^{\star}} \quad ; \quad T_{\phi_{2}, \alpha}(P)=\bullet_{\bullet P_{\alpha}^{\sqcup}}^{P_{\alpha}^{\star}} .
$$

On the other hand, if $\alpha \in \operatorname{re}(P)$,

$$
T_{\phi_{r e}, \alpha}(P)=-\bullet_{\bullet P_{\alpha}^{*}}^{P_{\alpha}^{\sqcup}} \quad ; \quad T_{\phi_{2}, \alpha}(P)=\bigodot_{P_{\alpha}^{\sqcup}}^{P_{\alpha}^{\star}} .
$$


To see that $\mathfrak{T}_{\phi_{r e}}$ is not a dissection compatible Hopf algebra of $\mathcal{T}^{\bullet}(R)$, consider the polynomial dissection pair

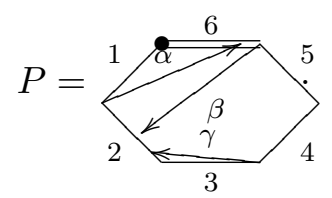

In $\mathfrak{T}_{\phi_{r e}}$, the generator

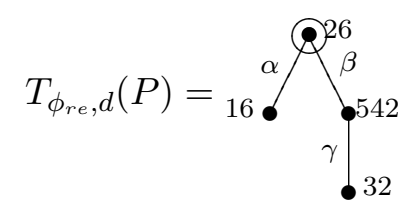

Consider the admissible cut $c=\{\beta\}$. The corresponding summand of the coproduct is

$$
\Delta_{c}\left(T_{\phi_{r e}, d}(P)\right)=\bullet^{26} \otimes \bigodot_{32}^{542}
$$

While $\bigodot_{16}^{26}=T_{\phi_{r e}, \alpha}(126), \bigodot_{32}^{542}=T_{\phi_{2}, \gamma}(5432)$. However, the generator $T_{\phi_{2}, \gamma}(5432) \notin \mathfrak{T}_{\phi_{r e}}$.

Lemma 42. The algebra $\mathfrak{T}_{\phi_{r e}}$ is not a dissection compatible Hopf algebra.

Proof. In fact, I show that $\mathfrak{T}_{\phi_{r e}}$ is not a Hopf algebra at all. Consider the generator $T_{\phi_{r e}, d}(P) \in \mathfrak{T}_{\phi_{r e}}$, and an admissible cut consisting of a single arrow, $c=\alpha \notin r e(P)$. The dissection $d \in D(P)$ can be decomposed into the sets

$$
d=d^{\bullet} \cup c \cup d^{\sqcup}
$$

where $d^{\bullet} \in D\left(P_{\alpha}^{\bullet}\right)$ and $d^{\sqcup} \in D\left(P_{\alpha}^{\sqcup}\right)$. Since $c=\alpha \notin r e(P)$, the root side of $P^{\sqcup}$ does not correspond to the root side of $P$. Furthermore, $d^{\sqcup} \cap \operatorname{re}(P)=\emptyset$. Thus, the above discussion shows that the corresponding summand in the coproduct is

$$
\Delta_{c}\left(T_{d}(P)\right)=T_{\phi_{r e}, d^{\bullet}}\left(P_{\alpha}^{\bullet}\right) \otimes T_{\phi_{2}, d^{\sqcup}}\left(P_{\alpha}^{\sqcup}\right) .
$$

If $d^{\sqcup} \cap \operatorname{re}\left(P_{\alpha}^{\sqcup}\right) \neq \emptyset$, then $T_{\phi_{2}, d^{\sqcup}}\left(P_{\alpha}^{\sqcup}\right) \notin \mathfrak{T}_{\phi_{r e}}$, and $\Delta\left(T_{d}(P)\right) \notin \mathfrak{T}_{\phi_{r e}} \otimes \mathfrak{T}_{\phi_{r e}}$. 
A class of differentials on $\mathcal{P}_{\bullet}^{(\star)}(R)$,

$$
\left\{\partial_{\phi} \mid \mathfrak{T}_{\phi} \text { dissection compatible Hopf algebra }\right\}
$$

defines a class of bar complexes $\left\{B_{\partial_{\phi}}\left(\mathcal{P}_{\bullet}^{(\star)}(R)\right)\right\}$. Continuing to generalize the construction of [7], I associate to each $R$-deco polygon an element of each $B_{\partial_{\phi}}\left(\mathcal{P}_{\bullet}^{(\star)}(R)\right)$.

Definition 43. If $\mathfrak{T}_{\phi}$ defines a differential $\partial_{\phi}$, define

$$
\begin{aligned}
\Lambda_{\phi} \quad: V_{\bullet}(R) & \rightarrow B_{\partial_{\phi}}\left(\mathcal{P}_{\bullet}^{(\star)}(R)\right) \\
P & \rightarrow \sum_{d \in D(P)} \Lambda\left(T_{\phi, d}(P)\right) .
\end{aligned}
$$

There is a natural way of identifying a subalgebra of $\bar{T}(V(R))$ that is generated by $R$-deco polygons

Definition 44. A polygon algebra defined by the dual tree generating set $\phi$ is

$$
B_{\phi}=\mathbb{Q}\left[\left\{\Lambda_{\phi}(P) \mid P R-\operatorname{deco}\right\}\right] .
$$

Its generators are in one to one correspondence with $R$-deco polygons.

In general, $B_{\phi}$ is a subalgebra of $\bar{T}((V(R))$

Proposition 45. If the dual tree generating set $\phi$ defines a dissection compatible Hopf algebra, then

$$
B_{\phi}=\mathbb{Q}\left[\left\{\Lambda_{\phi}(P) \mid P R-\text { deco polygon }\right\}\right]
$$

is a sub-Hopf algebra of $\bar{T}(V(R))$.

Proof. This is evident from the fact that the linearization map $\Lambda: \mathcal{T}^{\bullet}(R) \rightarrow$ $T\left(V_{\bullet}(R)\right)$ is a Hopf algebra homomorphism.

For $\mathfrak{T}_{\phi}$ a dissection compatible Hopf algebra, I show that $\Lambda_{\phi}(P)$ is contained in the 0th cocycles $B_{\partial_{\phi}}$, that is, $\Lambda_{\phi}(P) \in H^{0}\left(B_{\partial}\left(\mathcal{P}_{\bullet}^{(\star)}(R)\right)\right.$.

Theorem 46. Let the dual tree generating set $\phi$ generate a dissection compatible Hopf algebra $\mathfrak{T}_{\phi}$. Let $\partial_{\phi}$ be the associated differential. For $P$ an $R$ deco polygon, $\Lambda_{\phi}(P)$ is a 0 cocycle of $D_{1}+D_{2}$ in $B_{\partial_{\phi}}\left(\mathcal{P}_{\bullet}^{(\star)}(R)\right)$. 
Proof. Let $\pi_{k}$ be the projection of $\Lambda_{\phi}(P)$ onto its $k$ th direct sum component,

$$
\pi_{k}: \Lambda_{\phi}(P) \rightarrow \mathcal{P}_{\bullet}^{(\star)}(R)^{\mid k}
$$

The elements of $\Lambda_{\phi}(P) \in \bar{T}\left(\mathcal{P}_{\bullet}^{(1)}(R)\right)$. In general, if all $a_{i} \in P_{\bullet}^{(1)}(R)$, the differentials $D_{1}$ and $D_{2}$ are

$$
\begin{aligned}
& D_{1}\left(\left[a_{1}|\cdots| a_{n}\right]\right)=\sum_{i=1}^{n-1}-\left[a_{1}|\cdots| a_{i} \wedge a_{i+1}|\cdots| a_{n}\right] \\
& D_{2}\left(\left[a_{1}|\cdots| a_{n}\right]\right)=\sum_{j=1}^{n}\left[a_{1}|\cdots| \partial_{\phi}\left(a_{j}\right)|\cdots| a_{n}\right]
\end{aligned}
$$

Let $P$ be a polygon of weight $n$. The term $\pi_{n} \circ \Lambda_{\phi}(P)$ is a sum of $n$ fold tensors of 2-gons. Therefore $D_{2}\left(\pi_{n} \circ \Lambda_{\phi}(P)\right)=0$. Furthermore, the term $D_{1}\left(\pi_{1} \circ \Lambda_{\phi}(P)\right)=0$ by construction.

For $d \in D(P)$ with $|d|=k-1$, the dual tree $T_{\phi, d}(P)$ has $k$ vertexes, labeled by the set $\left\{P_{d}^{1}, \ldots, P_{d}^{k}\right\}$. Write

$$
\Lambda\left(T_{\phi, d}(P)\right)=\operatorname{sign}_{\phi}(d) \sum_{\text {Lin. }\left(T_{\phi, d}(P)\right)}\left[P_{d}^{\lambda_{1}}|\ldots| P_{d}^{\lambda_{k}}\right] .
$$

Comparing $D_{1}\left(\pi_{k} \circ \Lambda_{\phi}(P)\right)$ to $D_{2}\left(\pi_{k-1} \circ \Lambda_{\phi}(P)\right)$ for $k \in\{2, \ldots, n\}$ gives the expressions

$$
\begin{aligned}
& D_{1}\left(\pi_{k} \circ \Lambda_{\phi}(P)\right) \\
& \quad=\sum_{\substack{d \in D(P) \operatorname{Lin} .\left(T_{\phi, d}(P)\right) \\
|d|=k-1}} \sum_{i=1}^{k}-\operatorname{sign}_{\phi}(d)\left[P_{d}^{\lambda_{1}}|\ldots| P_{d}^{\lambda_{i}} \wedge P_{d}^{\lambda_{i+1}}|\ldots| P_{d}^{\lambda_{k}}\right]
\end{aligned}
$$

and

$$
\begin{aligned}
& D_{2}\left(\pi_{k-1} \circ \Lambda_{\phi}(P)\right) \\
& \quad=\sum_{\substack{d^{\prime} \in D(P) \operatorname{Lin} .\left(T_{\phi, d^{\prime}}(P)\right) \\
\left|d^{\prime}\right|=k-2}} \sum_{i=1}^{k-1} \operatorname{sign}_{\phi}\left(d^{\prime}\right)\left[P_{d^{\prime}}^{\lambda_{1}^{\prime}}|\cdots| \partial_{\phi} P_{d^{\prime}}^{\lambda_{i}^{\prime}}|\cdots| P_{d^{\prime}}^{\lambda_{k}^{\prime}}\right] .
\end{aligned}
$$

If $P_{d}^{\lambda_{i}}$ and $P_{d}^{\lambda_{i+1}}$ are not adjacent in $T_{\phi, d}(P)$, then there exists a unique partial order preserving linearization $\rho \in \operatorname{Lin}\left(T_{\phi, d}(P)\right)$ that switches only 
the order in which those two terms are written: $P_{d}^{\lambda_{i}}=P_{d}^{\rho_{i+1}}, P_{d}^{\lambda_{i+1}}=P_{d}^{\rho_{i}}$ and $P_{d}^{\lambda_{j}}=P_{d}^{\rho_{j}}$ if $j \notin\{i, i+1\}$. Therefore the terms

$$
\left[P_{d}^{\lambda_{1}}|\cdots| P_{d}^{\lambda_{i}} \wedge P_{d}^{\lambda_{i+1}}|\cdots| P_{d}^{\lambda_{k}}\right]+\left[P_{d}^{\rho_{1}}|\ldots| P_{d}^{\rho_{i}} \wedge P_{d}^{\rho_{i+1}}|\cdots| P_{d}^{\rho_{k}}\right]=0
$$

in the sum for $D_{1}$. In the remaining terms for $D_{1}$, the polygons $P_{d}^{\lambda_{i}}$ is adjacent to $P_{d}^{\lambda_{i+1}}$.

Consider $d \in D(P)$ such that $|d|=k-1$ and $d^{\prime}=d \backslash \alpha$. For each such pair of dissections, $\alpha$ is a dissecting arrow of $P_{d^{\prime}}^{\lambda_{i}^{\prime}}$ for some $i$. Therefore, the term

$$
\partial_{\phi}\left(P_{d^{\prime}}^{\lambda_{i}^{\prime}}\right)=\sum_{\alpha \in D\left(P_{d^{\prime}}^{\lambda_{i}^{\prime}}\right)} \operatorname{sign}_{\phi}(\alpha)\left(P_{d^{\prime}}^{\lambda_{i}^{\prime}}\right)_{\alpha}^{1} \wedge\left(P_{d^{\prime}}^{\lambda_{i}^{\prime}}\right)_{\alpha}^{2}
$$

appears in the expression for $D_{2}$. The polygons $\left(P_{d^{\prime}}^{\lambda_{i}^{\prime}}\right)_{\alpha}^{1}$ and $\left(P_{d^{\prime}}^{\lambda_{i}^{\prime}}\right)_{\alpha}^{2}$ are adjacent in $T_{\phi, d}(P)$. There is a unique linear order of $T_{\phi, d}(P), \lambda$, such that

$$
P_{d}^{\lambda_{j}}=\left\{\begin{array}{l}
P_{d^{\prime}}^{\lambda_{j}^{\prime}} \text { if } j<i \\
\left(P_{d^{\prime}}^{\lambda_{j}^{\prime}}\right)_{\alpha}^{1} \text { if } j=i \\
\left(P_{d^{\prime}}^{\lambda_{i}^{\prime}}\right)_{\alpha}^{2} \text { if } j=i+1 \\
P_{d}^{\lambda_{j-1}^{\prime}} \text { if } j>i+1
\end{array} .\right.
$$

That is

$$
\left[P_{d}^{\lambda_{1}^{\prime}}|\cdots|\left(P_{d^{\prime}}^{\lambda_{i}^{\prime}}\right)_{\alpha}^{1} \wedge\left(P_{d^{\prime}}^{\lambda_{i}^{\prime}}\right)_{\alpha}^{2}|\cdots| P_{d}^{\lambda_{k}^{\prime}}\right]=\left[P_{d}^{\lambda_{1}}|\cdots| P_{d}^{\lambda_{i}} \wedge P_{d}^{\lambda_{i+1}}|\cdots| P_{d}^{\lambda_{k}}\right]
$$

Since the right-hand side appears with the sign $\left(-\operatorname{sign}_{\phi}(d)\right)$ in $D_{1}\left(\Lambda_{\phi}(P)\right)$ and the left-hand side with the $\operatorname{sign} \operatorname{sign}_{\phi}\left(d^{\prime}\right) \operatorname{sign}_{\phi}(\alpha)$ in $D_{2}\left(\Lambda_{\phi}(P)\right)$ these terms cancel.

The coproduct on the Hopf algebras $B_{\phi}$ has a particularly nice form. If the dual tree generating set $\phi$ generates a dissection compatible Hopf algebra $\mathfrak{T}_{\phi}$, the duality between dissections $d \in D(P)$ and edges of a generator $T_{\phi, d}(P) \in \phi$ gives a concept of an admissible dissection for the polygon $P$.

Lemma 47. Let $\mathfrak{T}_{\phi}$ be a dissection compatible Hopf algebra. The following are equivalent:

(1) The dissection $c \in D(P)$ has a generator, $T_{\phi, c}(P)$, with only leaf and root vertexes labeled $\left\{P_{c}^{1}, \ldots, P_{c}^{|c|+1}\right\}$. 
(2) The dissection $c \in D(P)$ is an admissible cut of $T_{\phi, d}(P)$, for any $d=c \cup\left(\bigcup_{1}^{n} d_{i}\right)$. Furthermore,

$$
\Delta_{c}\left(T_{\phi, d}(P)\right)=\operatorname{sign}_{\phi}(c) \prod_{j=1}^{m} T_{\phi, d_{j}}\left(P_{c}^{j}\right) \otimes \prod_{k=m+1}^{|c|+1} T_{\phi, d_{k}}\left(P_{c}^{k}\right) .
$$

Proof. For $2 \Rightarrow 1$, note that from the definition of admissible cut, if $c$ is an admissible cut of $T_{\phi, d}(P)$, the generator $T_{\phi, c}(P)$ has only root and leaf vertexes. The vertex labels $\left\{P_{c}^{i}\right\}$ come from the fact that $\mathfrak{T}_{\phi}$ is a dissection compatible Hopf algebra.

For $1 \Rightarrow 2$, let $\left\{P_{c}^{1}, \ldots, P_{c}^{|c|+1}\right\}$ be the $R$-deco polygons decorating $T_{\phi, c}(P)$. For $c \subseteq d$, let $\left\{T_{d_{1}}, \ldots, T_{d_{|c|+1}}\right\}$ be the root and leaf subtrees of $T_{\phi, d}(P)$ formed by removing the edges in $c$. Since the subtrees $T_{d_{i}}$ are either only connected to initial vertexes of the edges in $c$, or only to the final vertexes, $c$ is an admissible cut of $T_{\phi, d}(P)$. Therefore,

$$
\Delta_{c}\left(T_{\phi, d}(P)\right)=\operatorname{sign}_{\phi}(c) \prod_{i=1}^{m} T_{d_{i}} \otimes \prod_{j=m+1}^{|c|+1} T_{d_{j}}
$$

The dissection $d \in D(P)$ can be written

$$
d=c \cup\left(\bigcup_{1}^{|c|+1} d_{i}\right)
$$

with $d_{i}$ corresponding to the edges in $T_{d_{i}}$. Since $\mathfrak{T}_{\phi}$ is a dissection compatible Hopf algebra, $d_{i} \in D\left(P_{i}\right)$ and $\operatorname{sign}_{\phi}\left(d_{i}\right) T_{d_{i}}=T_{\phi, d_{i}}\left(P_{c}^{i}\right)$, and $\operatorname{sign}_{\phi}(d)=$ $\operatorname{sign}_{\phi}(c) \prod_{i=1}^{|c|+1} \operatorname{sign}_{\phi}\left(d_{i}\right)$.

Definition 48. The dissection $c \in D(P)$ is an admissible dissection of $P$ in $\phi$ if the generator $T_{\phi, c}(P)$, has only leaf or root vertexes.

This definition is Hopf algebra (i.e., $\phi)$ specific. Consider two dissection compatible Hopf algebras, $\mathfrak{T}_{\phi}$ and $\mathfrak{T}_{\phi^{\prime}}$. An admissible cut of $T_{\phi, d}(P)$ need not be an admissible cut of $T_{\phi^{\prime}, d}(P)$. For instance, the dissection $d=\{\alpha, \beta\}$ of the polygon

$$
P=1 \underbrace{\frac{4}{\alpha / \beta} 3}_{2}
$$

is an admissible cut in $\mathfrak{T}_{\phi_{4}}$ but not in $\mathfrak{T}_{\phi_{2}}$. 
Theorem 49. Let $\mathfrak{T}_{\phi}$ be a dissection compatible Hopf algebra. For $c$ an admissible dissection of $P$ in $\phi$, let $\left\{P_{c}^{1}, \ldots, P_{c}^{m}\right\}$ be the labels of roots vertexes, and $\left\{P_{c}^{m+1}, \ldots, P_{c}^{|c|+1}\right\}$ the decorations of the leaf vertexes. Then

$$
\Delta\left(\Lambda_{\phi}(P)\right)=\sum_{c \text { admis. }} \amalg_{i=1}^{m} \Lambda_{\phi}\left(P_{c}^{i}\right) \otimes \amalg_{j=m+1}^{|c|+1} \Lambda_{\phi}\left(P_{c}^{j}\right) .
$$

Proof. Fix an admissible dissection $c$ of $P$ in $\mathfrak{T}_{\phi}$. By Lemma 47 and Definition 48, for any $d \in D(P)$, such that $c \subset d$, write $d=c \cup_{j=1}^{|c|+1} d_{i}$ with $d_{i} \in D\left(P_{c}^{i}\right)$ and

$$
\Delta T_{\phi, d}(P)=\sum_{c \text { admis. }} \prod_{i=1}^{m} T_{\phi, d_{i}}\left(P_{c}^{i}\right) \otimes \prod_{j=m+1}^{|c|+1} T_{\phi, d_{j}}\left(P_{c}^{j}\right)
$$

Write

$$
\Delta \circ \Lambda_{\phi}(P)=\Delta \circ \Lambda\left(\sum_{d \in D(P)} T_{\phi, d}(P)\right)=(\Lambda \otimes \Lambda) \circ \Delta\left(\sum_{d \in D(P)} T_{\phi, d}(P)\right)
$$

where the first equality comes from the definition of $\Lambda_{\phi}(P)$ and the second equality from Theorem 21. Expanding this,

$$
\begin{aligned}
(\Lambda & \otimes \Lambda) \Delta \sum_{d \in D(P)} T_{\phi, d}(P) \\
& =(\Lambda \otimes \Lambda)\left(\sum_{d \in D(P)} \sum_{c \subset d \text { admis. }} \prod_{i=1}^{m} T_{\phi, d_{i}}\left(P_{c}^{i}\right) \otimes \prod_{j=m+1}^{|c|+1} T_{\phi, d_{j}}\left(P_{c}^{j}\right)\right) .
\end{aligned}
$$

Reorganizing terms and changing the order of summation gives

$$
\begin{aligned}
& \sum_{c \text { admis. }} \amalg_{i=1}^{m} \sum_{d_{i} \in D\left(P_{c}^{i}\right)} \Lambda\left(T_{\phi, d_{i}}\left(P_{c}^{i}\right)\right) \otimes \amalg_{j=m+1}^{|c|+1} \sum_{d_{j} \in D\left(P_{c}^{j}\right)} \Lambda\left(T_{\phi, d_{j}}\left(P_{c}^{j}\right)\right) \\
& =\sum_{c \text { admis. }} \amalg_{i=1}^{m} \Lambda_{\phi}\left(P_{c}^{i}\right) \otimes \amalg_{j=m+1}^{|c|+1} \Lambda_{\phi}\left(P_{c}^{j}\right) .
\end{aligned}
$$

\subsection{Properties of almost compatible algebras}

In this subsection, I show that if the dual tree generating set $\psi$ defines an almost $\phi$ compatible algebra, $\mathfrak{T}_{\psi}$, the polygon algebra $B_{\psi}$ is a Hopf algebra, 
even if $\mathfrak{T}_{\psi}$ is not. First I show a relationship between the image of $\Lambda$ acting on general trees that differ on the orientation of the edges connecting certain vertexes.

Definition 50. Let $I$ be a subset of the edges of a tree $T$. Let $T^{I}$ be the tree obtained from $T$ by reversing the orientation of the edges in $I$.

Lemma 51. Let $T \in \mathcal{T}^{\bullet}(R)$ be a decorated multi-rooted tree. Let $I$ be a subset of $n$ edges of the tree $T$. Let $F=\prod_{i=1}^{n+1} t_{i}$ be the forest of multi-rooted trees created by removing the edges in $I$ in $T$. Then

$$
\sum_{J \subseteq I} \Lambda\left(T^{J}\right)=\amalg_{i=1}^{n+1} \Lambda\left(t_{i}\right)
$$

Remark 52. The set $I$ need not be an admissible cut of $T$.

Proof. Write $I=\left\{e_{1}, \ldots, e_{n}\right\}$ with $v_{j 1}$ and $v_{j 2}$ the endpoints of the edge $e_{j}$, such that $v_{j 1} \prec v_{j 2}$ in $T$. If $e_{j} \in J$ then $v_{j 2} \prec v_{j 1}$ in $T^{J}$, and $v_{j 1} \prec v_{j 2}$ if not.

If $t_{k}$ and $t_{l}$ are two trees in the forest $F$, the vertexes of $t_{k}$ are incomparable to the vertexes of $t_{l}$ in $F$. By construction, no tree $t_{k}$ has both $v_{j 1}$ and $v_{j 2}$ as vertexes, for any $j$. Group $\Lambda(F)$ into sums of those terms where $v_{j 1}$ is to the left of $v_{j 2}$ and sums of those where the opposite is true. The relative positions of $v_{j 2}$ and $v_{j 1}$ correspond to the two orientations of the edge $e_{j}$. Since there are two choices for each pair, this divides the terms of $\Lambda(F)$ into $2^{n}$ sums. This groups $\Lambda(F)$ into the sums in the statement of the lemma.

Example 53. (1) If $I=\{e\}$ is a single edge of a tree $T$, removing $e$ gives two subtrees, $\{R, P\}$. Under this notation,

$$
\Lambda(T)+\Lambda\left(T^{e}\right)=\Lambda(R) \amalg \Lambda(P)
$$

(2) Let $T$ be multi-rooted tree formed by connecting the trees in the forest $F=\prod_{i=1}^{n} T_{i}$ to a new root with label $s$ at the vertex $v_{i}$ of $T_{i}$. For $n=3$,

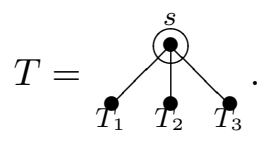


Define $I=\left\{e_{1}, e_{2}, e_{3}\right\}$ to be the edges that connect $s$ to $v_{i}$. Then

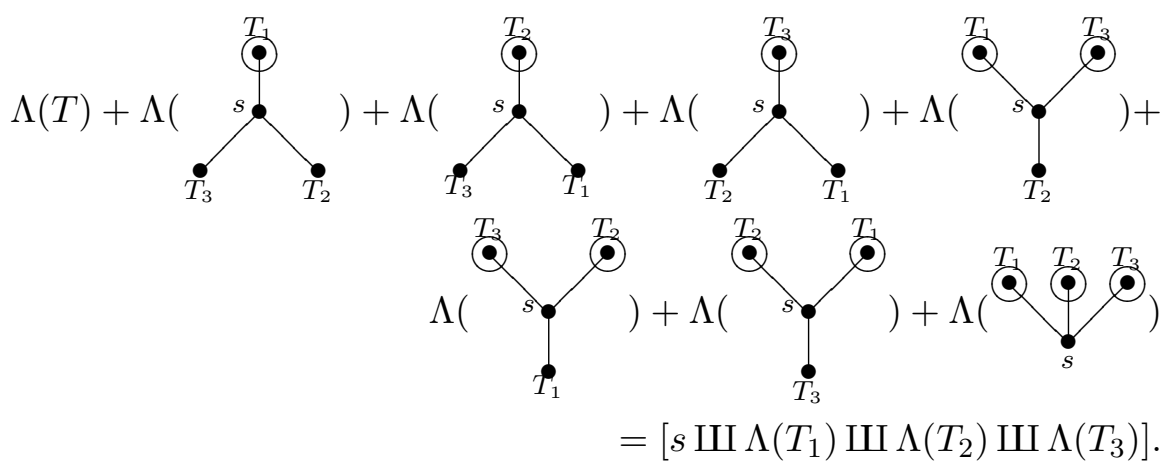

I use Lemma 51 to show a relationship between the polygon algebras $B_{\phi}$ and $B_{\psi}$, where $\mathfrak{T}_{\psi}$ is almost $\phi$ compatible, and $\mathfrak{T}_{\phi}$ is a dissection compatible Hopf algebra.

Theorem 54. Let $\phi$ and $\psi$ be two dual tree generating sets that define the same differential $\partial_{\phi}=\partial_{\psi}$. Let $\mathfrak{T}_{\phi}$ be a dissection compatible Hopf algebra and $\mathfrak{T}_{\psi}$ an almost $\phi$ compatible algebra. Let $\mathcal{S}$ be their difference set. Let $P$ be an $R$-deco polygon. For $d \in D(P)$, let $\left\{P_{d}^{1}, \ldots, P_{d}^{|d|+1}\right\}$ be the polygons decorating $T_{\phi, d}(P)$. Then

$$
\Lambda_{\psi}(P)-\Lambda_{\phi}(P)=\sum_{\substack{d \subset \mathcal{S} \\ \emptyset \neq d \in D(P)}}(-1)^{|d|} \operatorname{sign}_{\phi}(d) \amalg_{j=1}^{|d|+1} \Lambda_{\phi}\left(P_{d}^{j}\right) .
$$

for all $P \in V_{\bullet}(R)$.

Proof. For any $d \in D(P)$, let $I(d)=d \cap \mathcal{S}$. Write the generator

$$
T_{\phi, d}(P)=\operatorname{sign}_{\phi}(d) T \text {. }
$$

Using the notation from Lemma 51,

$$
T_{\phi, d}^{I(d)}(P)=\operatorname{sign}_{\phi}(d) T^{I(d)} .
$$

By the definition of almost $\phi$ compatible algebras,

$$
(-1)^{|I(d)|} T_{\phi, d}(P)=T_{\psi, d}^{I(d)}(P)
$$

and

$$
T_{\phi, d}(P)=T_{\psi, d}(P) \Leftrightarrow I(d)=\emptyset .
$$


Write the left-hand side of (1.10) as

$$
\sum_{d^{\prime} \in D(P)}\left((-1)^{\left|I\left(d^{\prime}\right)\right|} \Lambda\left(T_{\phi, d^{\prime}}^{I\left(d^{\prime}\right)}(P)\right)-\Lambda\left(T_{\phi, d^{\prime}}^{\emptyset}(P)\right)\right) .
$$

By Equation (1.11), I can ignore the dissections $d^{\prime}$ that do not intersect the set $\mathcal{S}$,

$$
\sum_{d^{\prime} \in D(P), I\left(d^{\prime}\right) \neq \emptyset}\left((-1)^{\left|I\left(d^{\prime}\right)\right|} \Lambda\left(T_{\phi, d^{\prime}}^{I\left(d^{\prime}\right)}(P)\right)-\Lambda\left(T_{\phi, d^{\prime}}^{\emptyset}(P)\right)\right) .
$$

Write the right-hand side of (1.10) as

$$
\sum_{\substack{d \subset \mathcal{S} \\ \emptyset \neq d \in D(P)}}(-1)^{|d|} \operatorname{sign}_{\phi}(d) \amalg_{j=1}^{|d|+1} \sum_{d_{j} \in D\left(P_{d}^{j}\right)} \Lambda\left(T_{\phi, d_{j}}\left(P_{d}^{j}\right)\right)
$$

Since $\mathfrak{T}_{\phi}$ is a dissection compatible Hopf algebra, write any subdissection $d \subset d^{\prime} \in D(P)$, as $d^{\prime}=d \cup\left(\cup_{j=1}^{|d|+1} d_{j}\right)$ with $d_{j} \in D\left(P_{d}^{j}\right)$. The forest

$$
\prod_{j=1}^{|d|+1} T_{\phi, d_{j}}\left(P_{d}^{j}\right)
$$

comes from to cutting $T_{\phi, d^{\prime}}(P)$ at the edges corresponding to $d$. Furthermore,

$$
\operatorname{sign}_{\phi}\left(P, d^{\prime}\right)=\operatorname{sign}_{\phi}(P, d)\left(\prod_{j=1}^{d+1} \operatorname{sign}_{\phi}\left(P_{d}^{j}, d_{j}\right)\right)
$$

Using Lemma 51, rewrite Equation (1.13) as

$$
\sum_{\substack{d \subset \mathcal{S} \\ \emptyset \neq d \in D(P)}} \sum_{d \subseteq d^{\prime} \in D(P)}(-1)^{|d|}\left(\sum_{\delta \subseteq d} \Lambda\left(T_{\phi, d^{\prime}}^{\delta}(P)\right)\right) .
$$


I do not consider dissections $d^{\prime}$ such that $d^{\prime} \cap \mathcal{S}=I\left(d^{\prime}\right)=\emptyset$, since this implies $\delta=d=0$. I reorganize the expression in (1.14) to

$$
\begin{aligned}
& \sum_{\substack{d^{\prime} \in D(P) \\
I\left(d^{\prime}\right) \neq \emptyset}} \sum_{\substack{\phi \neq d \subseteq I\left(d^{\prime}\right) \\
\delta \subseteq d}}(-1)^{|d|} \Lambda\left(T_{\phi, d^{\prime}}^{\delta}(P)\right) \\
& =\sum_{\substack{d^{\prime} \in D(P) \\
I\left(d^{\prime}\right) \neq \emptyset}} \sum_{\delta} \sum_{i=\max \{1,|\delta|\}}^{\left|I\left(d^{\prime}\right)\right|}(-1)^{i}\left(\begin{array}{c}
\left|I\left(d^{\prime}\right) \backslash \delta\right| \\
i-|\delta|
\end{array}\right) \Lambda\left(T_{\phi, d^{\prime}}^{\delta}(P)\right) .
\end{aligned}
$$

For any fixed $\delta \notin\left\{\emptyset, I\left(d^{\prime}\right)\right\}$, this expression vanishes, leaving

$$
\sum_{\substack{d^{\prime} \in D(P) \\ I\left(d^{\prime}\right) \neq \emptyset}}\left((-1)^{\left|I\left(d^{\prime}\right)\right|} \Lambda\left(T_{\phi, d^{\prime}}^{I\left(d^{\prime}\right)}(P)\right)-\Lambda\left(T_{\phi, d^{\prime}}^{\emptyset}(P)\right)\right)
$$

to match (1.12). The first term corresponds to the case where $\delta=I\left(d^{\prime}\right)$. The second term corresponds to the case, where $\delta=\emptyset$.

The following corollary shows that that $B_{\phi}$ and $B_{\psi}$ are isomorphic as Hopf algebras. The generators $\left\{\Lambda_{\phi}(P)\right\}$ and $\left\{\Lambda_{\psi}(P)\right\}$ define different bases of this vector space underlying $B_{\phi}$.

Corollary 55. Let $\phi, \psi$ and $\mathcal{S}$ be as in Theorem 54. Then $B_{\psi} \simeq B_{\phi}$ as Hopf algebras

Proof. The product and coproduct structure on $B_{\psi}$ is induced by the product and coproduct structures on $B_{\phi}$ and Equation (1.10). Therefore, $B_{\psi}$ is a Hopf algebra. In fact, Equation (1.10) shows that every generator, $\Lambda_{\psi}(P)$ of $B_{\psi}$, can be written in terms of sums of shuffles of generators of $B_{\phi}$. It remains to show that the map defined by this equation can be inverted.

By Theorem 54, write

$$
\Lambda_{\phi}(P)=\Lambda_{\psi}(P)-\sum_{\substack{d \subset \mathcal{S} \\ \emptyset \neq d \in D(P)}}(-1)^{|d|} \operatorname{sign}_{\phi}(d) \amalg_{j=1}^{|d|+1} \Lambda_{\phi}\left(P_{d}^{j}\right) .
$$

where $\left\{P_{d}^{1}, \ldots, P_{d}^{|d|+1 \mid}\right\}$ is the set of polygons decorating $T_{\phi, d}(P)$ and $T_{\psi, d}$ $(P)$. If $P \in V_{1}(R)$, that is, it is a 2 -gon,

$$
\Lambda_{\phi}(P)=\Lambda_{\psi}(P)
$$


If $P \in V_{2}(R)$,

$$
\Lambda_{\phi}(P)=\Lambda_{\psi}(P)+\sum_{\substack{d \subset \mathcal{S} \\ \emptyset \neq d \in D(P)}} \operatorname{sign}_{\phi}(d) \Lambda_{\phi}\left(P_{d}^{1}\right) \amalg \Lambda_{\phi}\left(P_{d}^{2}\right)
$$

where $P_{d}^{1}, P_{d}^{2} \in V_{1}(R)$. Therefore,

$$
\Lambda_{\phi}(P)=\Lambda_{\psi}(P)+\sum_{\substack{d \subset \mathcal{S} \\ \emptyset \neq d \in D(P)}} \operatorname{sign}_{\phi}(d) \Lambda_{\psi}\left(P_{d}^{1}\right) \amalg \Lambda_{\psi}\left(P_{d}^{2}\right) .
$$

By induction, suppose $\Lambda_{\phi}(P) \in B_{\psi}$ for all $P$ of weight less than $n$. If $P \in$ $V_{n}(R)$,

$$
\Lambda_{\phi}(P)=\Lambda_{\psi}(P)-\sum_{\substack{d \subset \mathcal{S} \\ \emptyset \neq d \in D(P)}}(-1)^{|d|} \operatorname{sign}_{\phi}(d) \amalg_{j=1}^{|d|+1} \Lambda_{\phi}\left(P_{d}^{j}\right) .
$$

Since each $P_{d}^{j}$ has weight less than $n, \Lambda_{\phi}\left(P_{d}^{j}\right) \in B_{\psi}$. Thus $\Lambda_{\phi}(P)$ can be written in terms of sums of shuffles of elements in $B_{\psi}$. Therefore, $\Lambda_{\phi}(P) \in$ $B_{\psi}$, and there is a one to one correspondence between the generators of $B_{\phi}$ and $B_{\psi}$.

Under these conditions, if, for all polygon dissection pairs $(P, d)$ every tree of the form $T_{\phi, d \cap \mathcal{S}}(P)$ is linear, then the result of Theorem 54 simplifies greatly.

Corollary 56. If in addition to the conditions for $\phi, \psi$ and $\mathcal{S}$ above, $T_{\phi, d \cap \mathcal{S}}(P)$ is a linear tree with sign, for all $d \in D(P)$, then

$$
\Lambda_{\phi}(P)-\Lambda_{\psi}(P)=\sum_{\alpha \in \mathcal{S}} \operatorname{sign}_{\phi}(\alpha) \Lambda_{\phi}\left(P_{\alpha}^{1}\right) \amalg \Lambda_{\psi}\left(P_{\alpha}^{2}\right),
$$

Proof. For $P$ an $R$-deco polygon, fix an $\alpha \in D(P)$ such that $\alpha \in \mathcal{S}$. Consider all $d \subset \mathcal{S}$ such that $\alpha$ is dual to the edge attached to the root in $T_{\phi, d}(P)$. Then the dissection $\{d \backslash \alpha\} \in D\left(P_{\alpha}^{2}\right)$. Let

$$
\rho_{\alpha}=\left\{\emptyset \neq d \in D(P) \mid d \subset \mathcal{S}, \alpha \in d, P_{\alpha}^{1} \text { root label of } T_{\phi, d}(P)\right\}
$$

be the set of all such $d$. Let $\left\{P_{d}^{1}, \ldots, P_{d}^{|d|+1}\right\}$ be the polygons decorating the generator $T_{\phi, d}(P)$ enumerated such that $P_{\alpha}^{1}=P_{d}^{1}$ labels the vertex of 
$T_{\phi, d}(P)$. From Theorem 54,

$$
\begin{aligned}
\Lambda_{\phi}(P)-\Lambda_{\psi}(P) & \\
= & -\sum_{\substack{d \subset \mathcal{S} \\
\emptyset \neq d \in D(P)}}(-1)^{|d|} \operatorname{sign}_{\phi}(d) \amalg_{j=1}^{|d|+1} \Lambda_{\phi}\left(P_{d}^{j}\right) \\
& =-\sum_{\alpha \in \mathcal{S}} \sum_{d \in \rho_{\alpha}}(-1)^{|d|} \operatorname{sign}_{\phi}(d) \Lambda_{\phi}\left(P_{\alpha}^{1}\right) \amalg_{j=2}^{|d|+1} \Lambda_{\phi}\left(P_{d}^{j}\right) .
\end{aligned}
$$

If $d=\alpha$, then $P_{d}^{2}=P_{\alpha}^{2}$. Break the sum in the last line of Equation (1.15) as

$$
\begin{aligned}
& \sum_{\alpha \in \mathcal{S} \cap D(P)} \operatorname{sign}_{\phi}(\alpha) \Lambda_{\phi}\left(P_{\alpha}^{1}\right) \amalg\left(\Lambda_{\phi}\left(P_{\alpha}^{2}\right)\right. \\
& \left.+\sum_{d \in \rho_{\alpha}, d \neq \alpha}(-1)^{|d|-1} \operatorname{sign}_{\phi}(d \backslash \alpha) \amalg_{j=2}^{|d|+1} \Lambda_{\phi}\left(P_{d}^{j}\right)\right) .
\end{aligned}
$$

Since $d \backslash \alpha \in D\left(P_{\alpha}^{2}\right)$, by Theorem 54

$$
\left.\Lambda_{\psi}\left(P_{\alpha}^{2}\right)=\Lambda_{\phi}\left(P_{\alpha}^{2}\right)+\sum_{d \in \rho_{\alpha}, d \neq \alpha}(-1)^{|d|-1} \operatorname{sign}_{\phi}(d \backslash \alpha)\right) \amalg_{j=2}^{|d|+1} \Lambda_{\phi}\left(P_{d}^{j}\right) .
$$

This gives

$$
\Lambda_{\phi}(P)-\Lambda_{\psi}(P)=\sum_{\alpha \in \mathcal{S} \in D(P)} \operatorname{sign}_{\phi}(\alpha) \Lambda_{\phi}\left(P_{\alpha}^{1}\right) \amalg \Lambda_{\psi}\left(P_{\alpha}^{2}\right) .
$$

Corollary 57. Under the same conditions as the previous corollary, one can also write

$$
\Lambda_{\phi}(P)-\Lambda_{\psi}(P)=\sum_{\alpha \in \mathcal{S} \cap D(P)} \operatorname{sign}_{\phi}(\alpha) \Lambda_{\psi}\left(P_{\alpha}^{1}\right) \amalg \Lambda_{\phi}\left(P_{\alpha}^{2}\right) .
$$

Proof. This result comes from the same argument as above, replacing the edge connected to the single root vertex of $T_{\phi, d}(P)$, for $d \subset \mathcal{S}$ with the edge connected to the single leaf vertex.

\section{Permutations of a polygon}

In this section, I examine the actions of $\sigma$ and $\tau$ on the Hopf algebra $\Lambda_{\phi_{2}}$. Recall that $\sigma$ and $\tau$ are linear automorphisms on $V_{\bullet}(R)$ such that 
for $P=12 \cdots n, \tau(P)=(n-1) \cdots 21 n$ reverses the orientation of $P$ and $\sigma(P)=2 \cdots n 1$ rotates the labels of the edges one position. Restricted to the sub-vector space $V_{n}(R),\left.\sigma\right|_{V_{n}(R)}$ and $\left.\tau\right|_{V_{n}(R)}$ generate the dihedral group $D_{2 n+2}$. I can extend $\sigma$ and $\tau$ to automorphisms of $B_{\phi_{2}}$ by defining $\left.\sigma\left(\Lambda_{\phi_{2}}(P)\right)=\Lambda_{\phi_{2}}(\sigma P)\right)$ and $\tau\left(\Lambda_{\phi_{2}}(P)\right)=\Lambda_{\phi_{2}}(\tau P)$. After defining relations between $\Lambda_{\phi_{2}}(\sigma P), \Lambda_{\phi_{2}}(\tau P)$ and $\Lambda_{\phi_{2}}(P)$, one can apply the coalgebra homomorphism

$$
\Phi: \Lambda\left(\mathfrak{T}_{2}\right) \rightarrow \mathcal{I}_{\bullet}(R)
$$

defined in [7] to establish relationships between iterated integrals with the appropriate dihedral action on the arguments.

\subsection{Order 2 generator of the dihedral group}

First I calculate $\Lambda_{\phi_{2}}(P) \pm \Lambda_{\phi_{2}}(\tau P)$. Since $\tau$ fixes the label of the root side of the polygon $P$, it is useful to examine an almost $\phi_{2}$ compatible algebra $\mathfrak{T}_{\psi}$ such that the difference set between $\phi_{2}$ and $\psi$ consists of arrows ending on the root side. This is exactly the algebra $\mathfrak{T}_{\phi_{r e}}$ discussed in Example 41.

Lemma 58. If $P$ is an $R$-deco polygon of weight $n$,

$$
\left.\Lambda_{\phi_{2}}(P)-\Lambda_{\phi_{r e}}(P)=\sum_{i=2}^{n} \Lambda_{\phi_{2}}\left(P_{i \alpha}^{\bullet-}\right)\right) \amalg \Lambda_{\phi_{r e}}\left(P_{i}^{\sqcup} \dot{ }\right) .
$$

Proof. For $i<j,{ }_{j} \alpha \in D\left(P_{i}^{\sqcup}\right)$ and ${ }_{i} \alpha \in D\left(P_{j}^{\bullet}\right)$. If $d=\left\{{ }_{i} \alpha,{ }_{j} \alpha\right\} \in D(P)$, $T_{\phi_{r e}, d}(P)$ and $T_{\phi_{2}, d}(P)$ are linear, as are the trees for and dissection $d \subset$ $r e(P)$. The arrows ${ }_{j} \alpha$ are forwards, so $\operatorname{sign}_{\phi_{2}}\left({ }_{j} \alpha\right)=1$. The result follows from Corollary 56.

Extend the action of $\tau$ to dissecting arrows and their associated sets of subpolygons.

Let $\alpha$ be a dissecting arrow of $P$, a polygon of weight $n$. Using the notation in Definition 3, if $\alpha \notin r e(P)$, write $\alpha={ }_{i} \alpha_{j}$ (for $j \neq n+1$ ). The map $\tau$ sends the polygon $P$ to $\tau(P)$ and the arrow ${ }_{i} \alpha_{j}$ to $\tau \alpha={ }_{n-i+2} \alpha_{n-j+1} \in$ $D(\tau(P))$. For a root ending arrow $\alpha={ }_{i} \alpha_{n+1} \in \operatorname{re}(P), \tau \alpha={ }_{n-i+2} \alpha_{n+1} \in$ $D(\tau(P))$. For a forward (backward) arrow $\alpha \notin r e(P)$, the arrow $\tau \alpha$ is 
backward (forward). The map $\tau$ take $r e(P)$ to itself. All arrows in $r e(P)$ are forward. The following is an example for $P$ of weight 5 .

Example 59. Recall that I use the shorthand ${ }_{i} \alpha$ to indicate the root ending arrow ${ }_{i} \alpha_{n+1} \in \operatorname{re}(P)$ (for $P \in V_{n}(R)$ ). Let $P=123456$, and $d=\left\{{ }_{3} \alpha, \beta\right\}$. Then $\tau d=\left\{{ }_{4} \alpha, \tau \beta\right\}$. Below are diagrams of $P$ and $\tau P$ with the dissections $d$ and $\tau d$ drawn in.
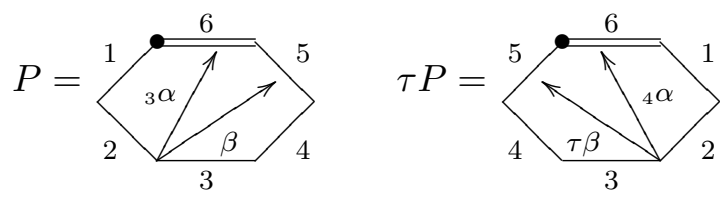

The arrows ${ }_{3} \alpha,{ }_{4} \alpha$ are in $r e(P)$ and $r e(\tau(P))$, respectively. The subpolygons associated to ${ }_{3} \alpha$ and ${ }_{4} \alpha$ are

$$
\tau\left(P_{3}^{\bullet}\right)=216=(\tau P)_{4}^{\sqcup} \quad ; \quad(\tau P)_{4}^{\bullet} \alpha=5436=\tau\left(P_{3 \alpha}^{\sqcup}\right)
$$

The subpolygons associated to $\beta$ and $\tau \beta$ are

$$
(\tau P)_{\tau \beta}^{\bullet}=5216=\tau\left(P_{\beta}^{\bullet-}\right) \quad ; \quad \tau P_{\beta}^{\sqcup}=435=(\tau P)_{\tau \beta}^{\sqcup} .
$$

For a general dissection of an arbitrary polygon, $d \in D(P)$, write $d \cap$ $r e(P)=\left\{i_{1} \alpha \ldots i_{j} \alpha\right\}$, with $i_{1}<i_{2} \cdots<i_{j}$. Let $\left\{P_{d}^{0}, \ldots, P_{d}^{|d|}\right\}$ be the set of polygons labeling the vertexes of $T_{\phi_{2}, d}(P)$ and $T_{\phi_{r e}, d}(P)$. Enumerate the set such that for $m \leq j, P_{d}^{m-1}$ and $P_{d}^{m}$ are adjacent, connected by $i_{m} \alpha$ with $P_{d}^{m-1} \prec P_{d}^{m}$ in $T_{\phi_{2}, d}(P)$ and $P_{d}^{m} \prec P_{d}^{m-1}$ in $T_{\phi_{r e}, d}(P)$. The set of polygons labeling $T_{\phi_{2}, \tau d}(\tau P)$ is $\left\{\tau P_{d}^{0}, \ldots, \tau P_{d}^{j}, P_{d}^{j+1}, \ldots, P_{d}^{|d|}\right\}$, with $\tau P_{m} \prec \tau P_{m-1}$ connected by $\tau_{i_{m}} \alpha$. If $d \cap \operatorname{re}(P)=\emptyset$ then $P_{0}$ is the label of the single root of all three generators. If $\beta \notin r e(P)$, write $d_{\beta}=d \cap D\left(P_{\beta}^{\sqcup}\right)$. The pruned subtrees of the admissible cut $c=\beta$ in $T_{\phi_{2}, d}(P), T_{\phi_{r e}, d}(P)$ and $T_{\phi_{2}, \tau d}(\tau P)$ are the same: $T_{\phi_{2}, d_{\beta}}\left(P_{\beta}^{\sqcup}\right)$ if $\beta$ is a forwards arrow, and $T_{\phi_{2}, d_{\beta}}\left(\tau P_{\beta}^{\sqcup}\right)$ if $\beta$ is a backwards arrow.

I summarize this in the following diagrams. Here $\beta, \gamma, \delta$, and $\epsilon$ are assumed to be forward arrows. For backwards arrows, replace $P_{\beta}^{\sqcup}$ with $\tau\left(P_{\beta}^{\sqcup}\right)$ 
and $d_{\beta}$ with $\tau d_{\beta}$.

$$
T_{\phi_{2}, d}(P)=\operatorname{sign}_{\phi_{2}}(d)
$$

The generators $T_{\phi_{2}, d}(P)$ and $T_{\phi_{r e}, d}(P)$ have different signs and different underlying tree structures, with labels $\left\{P_{d}^{0}, \ldots, P_{d}^{|d|}\right\}$. On the other hand, the generators $T_{\phi_{2}, \tau d}(\tau P)$ and $T_{\phi_{r e}, d}(P)$ have different signs, but the same underlying tree structure, if one exchanges $P_{d}^{m}$ with $\tau P_{d}^{m}$ for $m \leq j$. For any dissection $d \in D(P)$,

$$
\operatorname{sign}_{\phi_{r e}}(d)=(-1)^{|d \cap r e(P)|} \operatorname{sign}_{\phi_{2}}(d)=(-1)^{\sum_{\alpha \in d, \alpha \text { bw }} \chi(\alpha)}(-1)^{|d \cap r e(P)|}
$$

and

$$
\operatorname{sign}_{\phi_{2}}(\tau d)=(-1)^{\sum_{\alpha \in d \backslash r e(P), \alpha \text { fw }} \chi(\alpha)} .
$$

Recall that a coideal, $C$, of a coalgebra $(\mathcal{H}, \Delta, \varepsilon)$ has the structure

$$
\Delta(C) \subset \mathcal{H} \otimes C+C \otimes \mathcal{H}
$$

and $\varepsilon(C)=0$. It is a primitive coideal if

$$
\Delta\left(c_{i}\right)=1 \otimes c_{i}+c_{i} \otimes 1
$$

for all generators of $C$. 
Definition 60. Let $I_{n} \subset \mathcal{P}_{\bullet}^{(1)}(R)$ be the linear subspace generated by $\{P+$ $(-1)^{n} \tau P \mid P$ polygon of weight $\left.n\right\}$.

Note that $I_{1}=0$ is the trivial co-ideal. Each $I_{n}$ is a primitive co-ideal in $B_{\phi_{2}}$.

Definition 61. Define a set of quotient maps

$$
q_{n}: \bar{T}(V(R)) \rightarrow \bar{T}(V(R)) /\left(\sum_{k=1}^{n} I_{k}\right)
$$

Theorem 62. Let $P$ be an $R$-deco polygon of weight $n$. Let $q_{n}$ be the quotient map defined above. For $P \in V_{n}(R)$,

$$
\Lambda_{\phi_{r e}}(P)+(-1)^{n} \Lambda_{\phi_{2}}(\tau P) \in \operatorname{ker} q_{n}
$$

Proof. If $P$ is a polygon of weight $2, \Lambda_{\phi_{r e}}(P)-\Lambda_{\phi_{2}}(\tau P)=0$. For $P=a b c \in$ $V_{2}(R)$,

$$
\begin{aligned}
& \frac{c}{a / b} \\
& \Lambda_{\phi_{r e}}(P)=\quad P \quad+[b c \mid a b]-[b c \mid a c]-[a c \mid b a]
\end{aligned}
$$
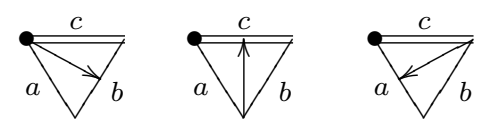

While for $\tau P$,

$$
\begin{gathered}
\sum_{b}^{c} \sum_{b}^{c} \sum_{a}^{c} \\
\Lambda_{\phi_{2}}(\tau P)={ }_{\tau P}
\end{gathered}
$$
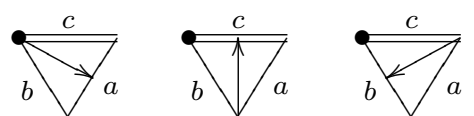

Therefore,

$$
\Lambda_{\phi_{r e}}(P)+\Lambda_{\phi_{2}}(\tau P)=P+\tau P \in I_{2} .
$$

Suppose the theorem holds for all $k<n$.

Let $P$ be an $R$-deco polygon of weight $n$. Consider the dissections $d \in$ $D(P)$, with $d \cap \operatorname{re}(P)=\left\{i_{1} \alpha, \ldots, i_{j} \alpha\right\}$. Let $\left\{P_{d}^{0}, \ldots, P_{d}^{|d|}\right\}$ be the set of polygons decorating $T_{\phi_{r e}, d}(P)$ and $T_{\phi_{2}, d}(P)$, with each $P_{d}^{i} \in V_{n_{i}}(R)$ with $\sum_{i=0}^{|d|}$ $n_{i}=n$. For $m \leq j$, the polygons $P_{d}^{m-1}$ and $P_{d}^{m}$ are adjacent, connected by $i_{m} \alpha$, and $P_{d}^{m} \prec P_{d}^{m-1}$ in $T_{\phi_{r e}, d}(P)$ and $P_{d}^{m-1} \prec P_{d}^{m}$ in $T_{\phi_{2}, d}(P)$. I define a series of trees (with sign) $\left\{T_{m, d}(P)\right\}$, with $m \leq j$ formed by replacing 
the polygons $\left\{P_{d}^{0}, \ldots, P_{d}^{m}\right\}$ in $T_{\phi_{r e}, d}(P)$ with the polygons $\left\{(-1)^{n_{0}} \tau P_{d}^{0}, \ldots\right.$, $\left.(-1)^{n_{m}} \tau P_{d}^{m}\right\}$. In this series, $T_{\phi_{r e}, d}(P)=T_{-1, d}(P)$. For example

$$
T_{0, d}(P)=\operatorname{sign}_{\phi_{r e}}(d)(-1)^{n_{0} T_{\phi_{2}, d_{\epsilon}}\left(P_{\epsilon}^{\sqcup}\right)} T_{T_{\phi_{2}, d_{\delta}}\left(P_{\delta}^{\sqcup}\right)}(P)=\operatorname{sign}_{\phi_{r e}}(d)(-1)^{n_{0}+n_{1}} T_{\phi_{2}, d_{\epsilon}}\left(P_{\epsilon}^{\sqcup}\right)
$$

For $m \leq j, \Lambda\left(T_{m-1, d}(P)+T_{m, d}(P)\right) \in \operatorname{ker} q_{n_{m}}$. The alternating sum,

$$
\sum_{m=0}^{j}(-1)^{m} \Lambda\left(T_{m-1, d}(P)+T_{m, d}(P)\right)=\Lambda\left(T_{\phi_{r e}, d}(P)+(-1)^{j} T_{j, d}(P)\right)
$$

is in $\operatorname{ker} q_{\sum_{m=1}^{j} n_{m}}$. Since

$$
\begin{aligned}
(-1)^{j} \operatorname{sign}\left(T_{j, d}\right) & =(-1)^{\sum_{i=0}^{j} n_{i}} \operatorname{sign}_{\phi_{2}}(d) \\
& =(-1)^{\sum_{i=0}^{j} n_{i}}(-1)^{\sum_{\alpha \in d, \alpha \text { bw }} \chi(\alpha)}=(-1)^{n} \operatorname{sign}_{\phi_{2}}(\tau d),
\end{aligned}
$$

for all $d \in D(P), d \neq \emptyset$,

$$
(-1)^{j} T_{j, d}(P)=(-1)^{n} T_{\phi_{2}, \tau d}(\tau P) .
$$

Applying $\Lambda$ on these trees and generators gives

$$
\Lambda\left(\sum_{\substack{d \in D(P) \\ d \neq \emptyset}} T_{\phi_{r e}, d}(P)+(-1)^{n} T_{\phi_{2}, \tau d}(\tau P)\right) \in \operatorname{ker}\left(q_{n-1}\right) .
$$

Writing $\Lambda\left(T_{\phi_{r e}, \emptyset}(P)\right)=[P]$ Equation (2.3) gives

$$
\Lambda_{\phi_{r e}}(P)-[P]+(-1)^{n}\left(\Lambda_{\phi_{2}}(\tau P)-[\tau P]\right) \in \operatorname{ker} q_{n-1},
$$


and

$$
\Lambda_{\phi_{r e}}(P)+(-1)^{n} \Lambda_{\phi_{2}}(\tau P) \in \operatorname{ker} q_{n}
$$

Combining Theorem 62 with Lemma 58 gives the following result.

Theorem 63. If $P$ is an $R$-deco polygon of weight $n$,

$$
q_{n}\left(\Lambda_{\phi_{2}}(P)+(-1)^{n} \Lambda_{\phi_{2}}(\tau P)\right)=q_{n}\left(\sum_{i=2}^{n-1}(-1)^{n-i} \Lambda_{\phi_{2}}\left(P_{i}^{\bullet} \alpha\right) \amalg \Lambda_{\phi_{2}}\left(\tau\left(P_{i \alpha}^{\sqcup}\right)\right)\right) .
$$

Proof. Let $P$ be a polygon of weight $n$. Apply $q_{n}$ to both sides of (2.1)

$$
q_{n}\left(\Lambda_{\phi_{2}}(P)-\Lambda_{\phi_{r e}}(P)\right)=q_{n}\left(\sum_{i=2}^{n} \Lambda_{\phi_{2}}\left(P_{i \alpha}^{\bullet}\right) \amalg \Lambda_{\phi_{r e}}\left(P_{i \alpha}^{\sqcup}\right)\right)
$$

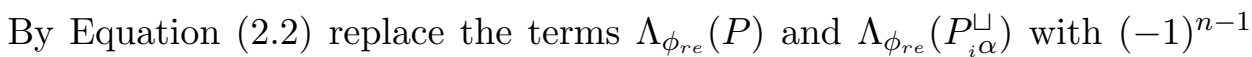
$\Lambda_{\phi_{2}}(\tau P)$ and $(-1)^{n-i} \Lambda_{\phi_{2}}\left(\tau P_{i}^{\sqcup}\right)$ to get

$$
q_{n}\left(\Lambda_{\phi_{2}}(P)+(-1)^{n} \Lambda_{\phi_{2}}(\tau P)\right)=q_{n}\left(\sum_{i=2}^{n}(-1)^{n-i} \Lambda_{\phi_{2}}\left(P_{i \alpha}^{\bullet}\right) \amalg \Lambda_{\phi_{2}}\left(\tau\left(P_{i}^{\sqcup} \dot{ }\right)\right)\right)
$$

as desired.

This show that $\Lambda_{\phi_{2}}(P)$ and $\Lambda_{\phi_{2}}(\tau P)$ can be compared up to a primitive coideal. This relation between decorated polygons of different orientation is reminiscent of a relation between iterated integrals on $R \subset \mathbb{C}^{\times}$. Recall that for iterated integrals, there is the relation [10]

$I\left(0 ; x_{1}, \ldots, x_{n} ; y\right) I\left(0 ; w_{1}, \ldots, w_{m} ; y\right)=I\left(0 ;\left(x_{1}, \ldots, x_{n}\right) \amalg\left(w_{1}, \ldots, w_{m}\right) ; y\right)$.

Lemma 64. For $r_{i} \in R$,

$$
\begin{aligned}
& I\left(0 ; r_{1}, \ldots, r_{n} ; r_{n+1}\right)+(-1)^{n} I\left(0 ; r_{n}, \ldots, r_{1} ; r_{n+1}\right) \\
& \quad=\sum_{i=2}^{n}(-1)^{n-i} I\left(0 ; r_{1}, \ldots, r_{i-1} ; r_{n+1}\right) I\left(0 ; r_{n}, \ldots, r_{i} ; r_{n+1}\right) .
\end{aligned}
$$


Proof. This proof is also presented in [10]. Rewrite the right hand side of (2.4) as

$$
\sum_{i=2}^{n}(-1)^{n-i} I\left(0 ;\left(r_{1}, \ldots, r_{i-1}\right) \amalg\left(r_{n}, \ldots, r_{i}\right) ; r_{n+1}\right) .
$$

For a fixed $i$ each term in the shuffle product in Equation (2.4) can be broken down into two groups, the terms where $r_{i-1}$ comes before $r_{i}$ and the terms where it comes after. By the alternating signs, the former cancel with a term in the shuffle

$$
I\left(0 ;\left(r_{1}, \ldots, r_{i}\right) \amalg\left(r_{n}, \ldots, r_{i+1}\right) ; r_{n+1}\right),
$$

and the latter in the shuffle

$$
I\left(0 ;\left(r_{1}, \ldots, r_{i-2}\right) \amalg\left(r_{n}, \ldots, r_{i}, r_{i-1}\right) ; r_{n+1}\right) .
$$

What remains are the terms (from $i=2)(-1)^{n} I\left(0 ; r_{n}, \ldots, r_{1} ; r_{n+1}\right)$ and (from $i=n) I\left(0 ; r_{1}, \ldots, r_{n} ; r_{n+1}\right)$, which are the terms on the left-hand side of (2.4).

Remark 65. The relationship expressed in (2.4) is exact on iterated integrals, while there is a relation only up to a primitive coideal on the level of polygons, as shown in Theorem 63. This is in contradiction to the conjecture by Gangl and Brown that relationships between dihedral symmetries of $R$-deco polygons can be expressed purely in terms of shuffle products of polygons of lower weights. It also shows that the coalgebra map between the bar elements associated to polygons and iterated integrals, (1.1)

$$
\Phi: B_{\phi_{2}} \rightarrow \mathcal{I}_{\bullet}(R)
$$

is not injective: the coideals $I_{n} \in \operatorname{ker} \Phi$ for $n \geq 1$.

\subsection{Order $n$ generator of the dihedral group}

In this subsection, I consider the rotation map, $\sigma$ on $\mathcal{P}_{\bullet}^{(\star)}(R)$ that sends the $R$-deco polygon $P$ to $\sigma P$. For $P=12 \ldots n, \sigma P=2 \ldots n 1$ is the polygon rotated clockwise, changing the root side. When restricted to $V_{n}(R),\left.\sigma\right|_{V_{n}(R)}$ is the order $n$ generator of the dihedral group. In order to examine this rotation, I work with $\partial_{\phi_{4}}$, which reflect the symmetry of the change, and relate the corresponding elements of the bar construction to $B_{\phi_{2}}$. 
2.2.1. Relating $\boldsymbol{B}_{\phi_{\mathbf{2}}}$ to $\boldsymbol{B}_{\phi_{4}}$ To understand the action of $\sigma$ on $I(R)$, one wants to calculate $\left.\Lambda_{\phi_{2}}(P)-\Lambda_{\phi_{2}}(\sigma P)\right)$. This is a difficult calculation, and it is easier to break down into intermediate steps. I use the results of the last section to relate the algebras $\Lambda_{\phi_{2}}(P)-\Lambda_{\phi_{4}}(P)$. I then study the action of $\sigma$ on the algebra $B_{\phi_{4}}$.

Definition 66. Let $b(P)=\{$ backwards arrows of $P\}$.

Recall that $\mathfrak{T}_{\phi_{4}}$ and $\mathfrak{T}_{\phi_{3}}$ are both dissection compatible Hopf algebras, and that $\phi_{3}$ and $\phi_{4}$ define the same differential. The difference set between the dual tree generating sets $\phi_{3}$ and $\phi_{4}$ is $\mathcal{S}=\bigcup_{P R \text {-deco }} b(P)$. The algebra $\mathfrak{T}_{\phi_{3}}$ is almost $\phi_{4}$ compatible.

Theorem 67. For $P$ an $R$-deco polygon of weight $n$, and $d \in D(P)$, let $\left\{P_{d}^{1}, \ldots, P_{d}^{|d|+1}\right\}$ be the set of polygons decorating the generator $T_{\phi_{4}, d}(P)$. Then, for the map $q_{n}$ as defined in Definition 61

$$
q_{n-1}\left(\Lambda_{\phi_{2}}(P)\right)=q_{n-1}\left(\Lambda_{\phi_{4}}(P)+\sum_{d \subseteq b(P)}(-1)^{|d|} \amalg_{j=1}^{|d|+1} \Lambda_{\phi_{4}}\left(P_{d}^{j}\right)\right) .
$$

Proof. For all arrows $d \subset b(P), \operatorname{sign}_{\phi_{4}}(d)=+1$. By Theorem 54

$$
\Lambda_{\phi_{3}}(P)=\Lambda_{\phi_{4}}(P)+\sum_{d \subseteq b(P)}(-1)^{|d|} \amalg_{j=1}^{|d|+1} \Lambda_{\phi_{4}}\left(P_{d}^{j}\right) .
$$

For $d \in D(P)$, with $|b(P) \cap d|=j$, let the set $\left\{P_{d}^{1}, \ldots, P_{d}^{|d|+1}\right\}$ decorate the vertexes of $T_{\phi_{3}, d}(P)$ and $T_{\phi_{4}, d}(P)$ enumerated $\left\{P_{d}^{1}, \ldots, P_{d}^{|d|+1}\right\}$ so that $\left\{P_{d}^{1}, \ldots, P_{d}^{j}\right\}$ decorate the terminal vertexes of the edges associated to a backwards arrow in $T_{\phi_{3}, d}(P)$. Then the set

$$
\left\{\tau P_{d}^{1}, \ldots, \tau P_{d}^{j}, P_{d}^{j+1}, \ldots, P_{d}^{|d|+1}\right\}
$$

decorates the vertexes of $T_{\phi_{2}, d}(P)$. The generators $T_{\phi_{2}, d}(P)$ and $T_{\phi_{4}, d}(P)$ have different signs, but the same underlying trees, with $P_{d}^{m}$ replacing $\tau P_{d}^{m}$ for $m \leq j$. Let $P_{d}^{i} \in V_{n_{i}}(R)$ with $\sum_{i=1}^{|d|+1} n_{i}=n$.

Recall that

$$
\operatorname{sign}_{\phi_{2}}(d)=(-1)^{\sum_{i=1}^{j} n_{i}} \quad \text { and } \quad \operatorname{sign}_{\phi_{3}}(d)=(-1)^{j} .
$$

Define a series of signed trees $\left\{T_{i, d}(P)\right\}, 1 \leq i \leq j$ by replacing the polygons $\left\{\tau P_{d}^{1}, \ldots, \tau P_{d}^{i}\right\}$ in $T_{\phi_{2}, d}(P)$ with the set $\left\{(-1)^{n_{1}} P_{1}, \ldots,(-1)^{n_{i}} P_{d}^{i}\right\}$. In this 
series, $T_{\phi_{2}, d}(P)=T_{0, d}(P), T_{j, d}(P)=(-1)^{j} T_{\phi_{3}, d}(P)$, and

$$
\Lambda\left(T_{i-1, d}(P)+T_{i, d}(P)\right)
$$

is in $\operatorname{ker}\left(q_{n_{i}}\right)$. The alternating sum

$$
\sum_{i=1}^{j}(-1)^{i-1} \Lambda\left(T_{i-1, d}(P)+T_{i, d}(P)\right)=\Lambda\left(T_{\phi_{2}, d}(P)-T_{\phi_{3}, d}(P)\right)
$$

is in $\operatorname{ker}\left(q_{\sum_{i=1}^{j} n_{i}}\right)$. Summing over all dissections $d \in D(P)$ gives

$$
\Lambda_{\phi_{2}}(P)-\Lambda_{\phi_{3}}(P) \in \operatorname{ker} q_{n-1}
$$

Plugging this into Equation (2.6) gives

$$
\left.q_{n-1}\left(\Lambda_{\phi_{2}}(P)\right)=q_{n-1}\left(\Lambda_{\phi_{4}}(P)\right)+\sum_{d \subseteq b(P)}(-1)^{|d|} \amalg_{j=1}^{|d|+1} \Lambda_{\phi_{4}}\left(P_{d}^{j}\right)\right)
$$

Example 68. Let $P_{2}=a b c, P_{3}=a b c d$ be $R$-deco polygons of weight 2 and 3. The following are the explicit calculations for $P_{2}$, abd $P_{3}$.

$$
d=\overbrace{b}^{c}
$$

Since $\operatorname{ker} q_{1}=0$, this is an exact relation:

$$
\left.\left.\Lambda_{\phi_{2}}\left(P_{2}\right)\right)-\Lambda_{\phi_{4}}\left(P_{2}\right)\right)=-\Lambda_{\phi_{4}}(a c) \amalg \Lambda_{\phi_{4}}(b a)
$$

For $\left.P_{3}, q_{3}\left(\Lambda_{\phi_{2}}\left(P_{3}\right)-\Lambda_{\phi_{4}}\left(P_{3}\right)\right)\right)=$

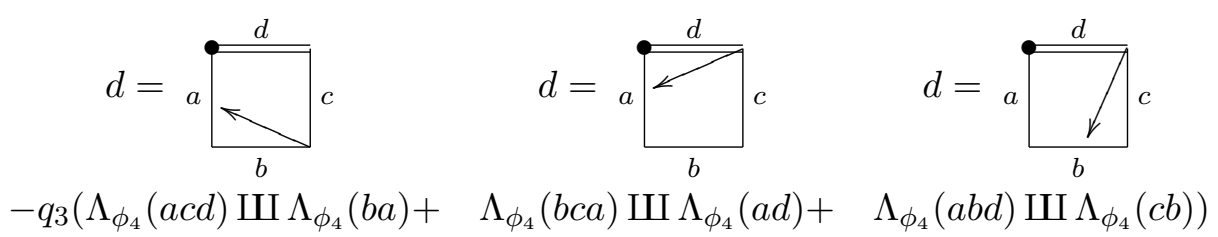




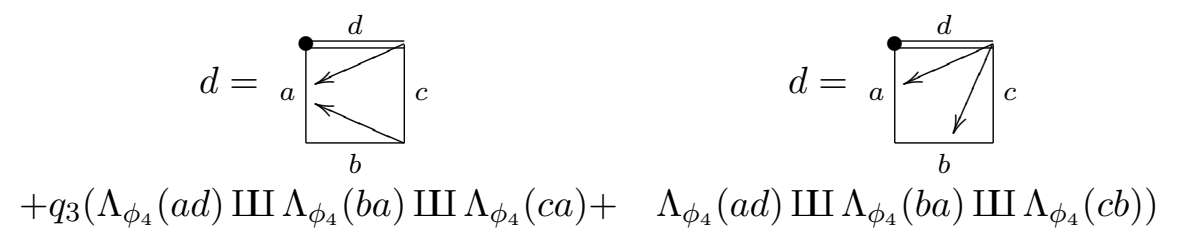

Since ker $q_{2} \neq 0$, computing the difference explicitly gives

$$
\begin{aligned}
\Lambda_{\phi_{2}}\left(P_{3}\right)-\Lambda_{\phi_{4}}\left(P_{3}\right)= & -\Lambda_{\phi_{4}}(a c d) \amalg \Lambda_{\phi_{4}}(b a)-\Lambda_{\phi_{4}}(b c a) \amalg \Lambda_{\phi_{4}}(a d) \\
& -\Lambda_{\phi_{4}}(a b d) \amalg \Lambda_{\phi_{4}}(c b)+\Lambda_{\phi_{4}}(a d) \amalg \Lambda_{\phi_{4}}(b a) \amalg \Lambda_{\phi_{4}}(c a) \\
& +\Lambda_{\phi_{4}}(a d) \amalg \Lambda_{\phi_{4}}(b a) \amalg \Lambda_{\phi_{4}}(c b)+[a d \mid b c a+c b a]
\end{aligned}
$$

The Hopf algebra $B_{\phi_{4}}$ is contained in $H^{0}\left(B_{\partial_{3}}\left(\mathcal{P}_{\bullet}^{(\star)}(R)\right)\right)$ by Theorem 46 .

2.2.2. Introducing a new symmetry. Instead of directly comparing $\Lambda_{\phi_{2}}(P)$ and $\Lambda_{\phi_{2}}(\sigma P)$ in this subsection, I compare $\Lambda_{\phi_{4}}(P)$ and $\Lambda_{\phi_{4}}(\sigma P)$. Theorem 67 then relates these terms to $\Lambda_{\phi_{2}}(P)$ and $\Lambda_{\phi_{2}}(\sigma P)$, respectively, as desired.

Definition 69. For $P$ the $R$-deco polygon $12 \ldots n$, let $(\sigma P)$ be the $R$-deco polygon $2 \ldots n 1$ with labels rotated one place in a clockwise direction.

Example 70. For the weight 3 polygon $P=1234$ one has

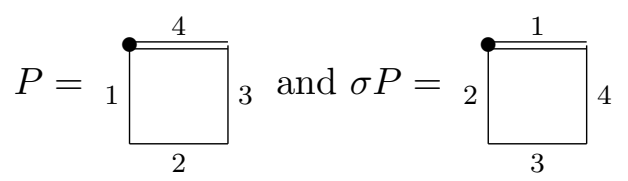

Example 71. For a weight 1 polygon, $P=a b, \sigma P=b a$, applying the map $\Phi$ from Equation (1.1)

$$
\begin{aligned}
\Phi\left(\Lambda_{\phi_{4}}(P)-\Lambda_{\phi_{4}}(\sigma P)\right) & =\Phi\left(\Lambda_{\phi_{2}}(P)-\Lambda_{\phi_{2}}(\sigma P)\right) \\
& =\mathbb{L i}_{1}\left(\frac{a}{b}\right)-\mathbb{L i}_{1}\left(\frac{b}{a}\right)=\ln (b)-\ln (a)
\end{aligned}
$$

The last equality holds up to a power of $i \pi$. 
Direct calculation shows that for $P=a b c$,

$$
\begin{aligned}
& \Lambda_{\phi_{4}}(P)-\Lambda_{\phi_{4}}(\sigma P) \\
& =P-\sigma P+[a b \amalg b c]-[b a \amalg c b]+[(a c-c a+b a-a b) \mid b c] \\
& \quad+[b a \mid(a c-c a+c b-b c)] .
\end{aligned}
$$

Applying Theorem 67 gives

$$
\begin{aligned}
& \Lambda_{\phi_{2}}(P)-\Lambda_{\phi_{2}}(\sigma P) \\
& =P-\sigma P+[a b \amalg b c]-[a c \amalg b a]+[(a c-c a+b a-a b) \mid b c] \\
& \quad+[b a \mid(a c-c a+c b-b c)] .
\end{aligned}
$$

Subsequent direct calculations get increasingly complex.

To calculate this relation for higher weight polygons, I examine the action of $\sigma$ on the dissecting arrows of an $R$-deco polygon $P$ of weight $n$. The rotation map $\sigma$ acts on dissecting arrows, rotating the starting vertex and ending edge one position backwards, as defined by the orientation of the polygon. Therefore, $\sigma\left({ }_{i} \alpha_{j}\right)={ }_{i-1} \alpha_{j-1}$ if $i$ or $j \neq 1, \sigma\left({ }_{1} \alpha_{j}\right)={ }_{n+1} \alpha_{j-1}$ and $\sigma\left({ }_{i} \alpha_{1}\right)={ }_{i-1} \alpha_{n+1}$.

Example 72. For the 4-gons $P$ and $\sigma P$, the dissecting arrows $\alpha$ and $\sigma \alpha$ are as follows:
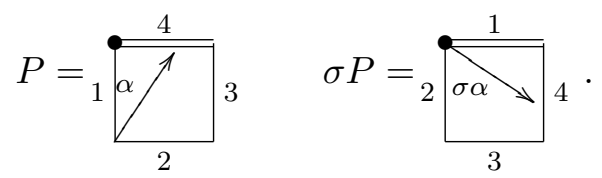

For a general $d \in D(P)$, write $d=\left\{\alpha_{1}, \ldots \alpha_{k}\right\}$ and $\sigma d=\left\{\sigma \alpha_{1}, \ldots, \sigma \alpha_{k}\right\}$. To compare $\Lambda_{\phi_{4}}(P)$ and $\Lambda_{\phi_{4}}(\sigma P)$, I start with dissections of $P$ with one arrow. There are two cases to consider.

(1) The dissecting arrow $\alpha$ starts at the first vertex. The first vertex is in both $P_{\alpha}^{r}$ and in $P_{\alpha}^{l}$. The associated subpolygons $P$ are related to the subpolygons of $\sigma P$ by

$$
\sigma\left(P_{\alpha}^{r}\right)=(\sigma P)_{\sigma \alpha}^{r} ; \quad \sigma\left(P_{\alpha}^{l}\right)=(\sigma P)_{\sigma \alpha}^{l}
$$


as illustrated in the following example:

$$
\begin{aligned}
& P={ }_{1} \stackrel{4}{\alpha} \rightarrow P_{\alpha}^{l}=34 ; P_{\alpha}^{r}=123 \\
& \sigma P={ }_{2}^{\frac{1}{\sigma \alpha}} \underset{3}{\frac{\sigma}{\sigma}} \rightarrow(\sigma P)_{\sigma \alpha}^{l}=43 ;(\sigma P)_{\sigma \alpha}^{r}=231
\end{aligned}
$$

(2) The dissecting arrow $\alpha$ does not start at the first vertex: There are three sub-cases.

(a) The dissecting arrow $\alpha$ ends on the first edge in $P$ (labeled 1). The first vertex is in $P_{\alpha}^{r}$. The dissected polygons of $P$ and $\sigma P$ are

$$
\sigma\left(P_{\alpha}^{r}\right)=(\sigma P)_{\sigma \alpha}^{r} ; \quad P_{\alpha}^{l}=(\sigma P)_{\sigma \alpha}^{l}
$$

as illustrated in the following example:

$$
\begin{gathered}
P=1 \underbrace{}_{2} \rightarrow P_{\alpha}^{l}=21 ; P_{\alpha}^{r}=134 \\
\sigma P=2 \underbrace{\frac{1}{\int_{\sigma \alpha}}}_{3} \rightarrow(\sigma P)_{\sigma \alpha}^{l}=21 ;(\sigma P)_{\sigma \alpha}^{r}=341
\end{gathered}
$$

(b) The dissecting arrow $\alpha$ ends on the root edge in $P$ (labeled $n$ ). The first vertex is in $P_{\alpha}^{l}$. The dissected polygons of $P$ and $\sigma P$ are

$$
P_{\alpha}^{r}=(\sigma P)_{\sigma \alpha}^{r} ; \quad \sigma\left(P_{\alpha}^{l}\right)=\sigma P_{\sigma \alpha}^{l}
$$

as illustrated in the following example:

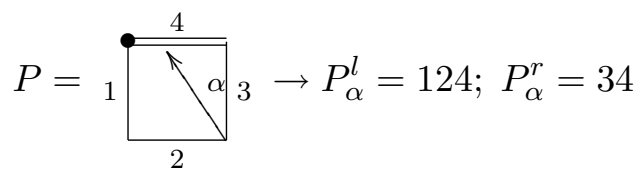




$$
\sigma P=2 \underbrace{}_{3} \frac{1}{\sigma \alpha /} 4 \rightarrow \sigma P_{\sigma \alpha}^{l}=241 ;(\sigma P)_{\sigma \alpha}^{r}=34
$$

(c) The dissecting arrow $\alpha$ ends on neither the first edge or root edge in $P$. The root vertex is in $P_{\alpha}^{l}$ if $\alpha$ is forward $\left(P_{\alpha}^{r}\right.$ if $\alpha$ backward). The dissected polygons of $P$ and $\sigma P$ are

$$
\sigma\left(P_{\alpha}^{l}\right)=(\sigma P)_{\sigma \alpha}^{l} ; \quad P_{\alpha}^{r}=(\sigma P)_{\sigma \alpha}^{r}
$$

if $\alpha$ is forwards, and

$$
\sigma\left(P_{\alpha}^{r}\right)=(\sigma P)_{\sigma \alpha}^{r} ; \quad P_{\alpha}^{l}=(\sigma P)_{\sigma \alpha}^{l}
$$

if $\alpha$ is backwards. This is illustrated in the following example:

$$
\begin{gathered}
P=1 \sum_{\frac{\alpha}{2}}^{4} \rightarrow P_{\alpha}^{l}=134 ; P_{\alpha}^{r}=23 \\
\sigma P=\underbrace{\frac{1}{\sigma \alpha}}_{3} \rightarrow(\sigma P)_{\sigma \alpha}^{l}=341 ;(\sigma P)_{\sigma \alpha}^{r}=23 .
\end{gathered}
$$

This exhaustively categorizes all possible dissecting arrows. I summarize the results as follows.

Lemma 73. Let $P$ be an $R$-deco polygon. For arrows of the form $1 \alpha_{j}$, starting at the first vertex, both subpolygons $P_{\alpha}^{r}$ and $P_{\alpha}^{l}$ contain the first vertex. The subpolygons of $P$ associated to a single dissecting arrow can be classified in the following way:

$$
(\sigma P)_{\sigma \alpha}^{r}= \begin{cases}\sigma\left(P_{\alpha}^{r}\right) & \text { if } P_{\alpha}^{r} \text { contains the first vertex of } P \\ P_{\alpha}^{r} & \text { otherwise }\end{cases}
$$

The same is true if $r$ is replaced with $l$.

To calculate the action of the operator $\sigma$ on the Hopf algebra $B_{\phi_{4}}$, I compare terms in the Hopf algebra $B_{\phi_{4}}$ to two new algebras $B_{\phi_{f v}}$ and $B_{\phi_{\sigma f v}}$, defined by new generating sets $\phi_{f v}$ and $\phi_{\sigma f v}$ that exploit the symmetries defined in Lemma 73. 
Definition 74. Let $f v(P)$ be set of arrows that start at the $f$ irst vertex of a polygon $P$. If $P \in V_{n}(R)$, write $f v(P)=\left\{\alpha_{2}, \ldots, \alpha_{n+1}\right\}$ where $\alpha_{i}$ ends at the $i$ th side. Define $\sigma f v(\sigma P)=\left\{\sigma \alpha_{2}, \ldots, \sigma \alpha_{n+1}\right\}$ to be the set of arrows that start at the $(n+1)^{t h}$ (last) vertex of $\sigma P$.

Define the generating sets $\phi_{f v}$ and $\phi_{\sigma f v}$ that define the same differential as $\phi_{4}$. Their difference set from $\phi_{4}$ is $\cup_{P} f v(P)$ and $\cup_{P} \sigma f v(P)$, respectively. Let $\mathfrak{T}_{\phi_{f v}}$ and $\mathfrak{T}_{\phi_{\sigma f v}}$ be the two almost $\phi_{4}$ compatible algebras defined by the dual tree generating sets $\phi_{f v}$ and $\phi_{\sigma f v}$.

Let $P$ be an $R$-deco polygon of weight $n$. Consider non-trivial dissections $d \in D(P)$, with $d \cap f v(P)=\left\{\alpha_{i_{1}} \cdots \alpha_{i_{j}}\right\}$, with $i_{1}<i_{2} \cdots<i_{j}$. Let $\left\{P_{d}^{0}, \ldots P_{d}^{|d|}\right\}$ be the set of polygons decorating $T_{\phi_{f v}, d}(P)$ and $T_{\phi_{4}, d}(P)$, with each $P_{d}^{i} \in V_{n_{i}}(R)$ with $\sum_{i=0}^{|d|} n_{i}=n$. For $m \leq j$, the polygons $P_{d}^{m-1}$ and $P_{d}^{m}$ are adjacent, connected by $\alpha_{i_{m}}$, and $P_{d}^{m-1} \prec P_{d}^{m}$ in $T_{\phi_{f v}, d}(P)$ and $P_{d}^{m} \prec P_{d}^{m-1}$ in $T_{\phi_{4}, d}(P)$. If $d \cap f v(P)=\emptyset, P_{d}^{0}$ is the subpolygon containing the first vertex of $P$. The set $\left\{\sigma P_{d}^{0}, \ldots, \sigma P_{d}^{j}, P_{d}^{j+1}, \ldots, P_{d}^{|d|}\right\}$ decorates the generator $T_{\phi_{4}, \sigma d}(\sigma P)$ with $\sigma P_{d}^{m} \prec \sigma P_{d}^{m-1}$, and $T_{\phi_{\sigma f v}, \sigma d}(\sigma P)$ with $\sigma P_{d}^{m-1} \prec \sigma P_{d}^{m}$. The subpolygons $\sigma P_{d}^{m-1}$ and $\sigma P_{d}^{m}$ are adjacent, connected by $\sigma \alpha_{i_{m}}$. If $d \cap f v(P)=\emptyset$, then $\sigma P_{0}$ is the subpolygon containing the last vertex of $\sigma P$. Recall that

$$
\operatorname{sign}\left(T_{f v, d}(P)\right)=\operatorname{sign}\left(T_{\sigma f v, \sigma d}(\sigma P)\right)=(-1)^{|d \cap f v(P)|},
$$

while $\operatorname{sign}\left(T_{\phi_{4}, d}(P)\right)=1$. If $\beta \notin f v(P)$, write $d_{\beta}=d \cap D\left(P_{\beta}^{\sqcup}\right)$. The polygon $P_{\beta}^{\sqcup}$ does not contain the first vertex of $P$, therefore $P_{\beta}^{\sqcup}=(\sigma P)_{\sigma \beta}^{\sqcup}$. By Lemma 73, the pruned subtrees corresponding to the admissible cut $c=\beta$ in $T_{\phi_{4}, d}(P)$, and $T_{\phi_{f v}, d}(P)$ are the same: $T_{\phi_{4}, d_{\beta}}\left(P_{\beta}^{\sqcup}\right)$. Similarly for $\sigma c=\sigma \beta$ in $T_{\phi_{\sigma f v}, \sigma d}(\sigma P)$ and $T_{\phi_{4}, \sigma d}(\sigma P)$ the pruned trees are both $T_{\phi_{4}, \sigma d_{\beta}}\left(\sigma P P_{\beta}^{\sqcup}\right)$. I summarize this in the following diagrams. Note that $P_{d}^{0}$ and $P_{d}^{j}$ need not be a root vertex of these generators. These trees are drawn without a root vertex specified.

$$
T_{\phi_{4}, d}(P)=T_{T_{4}, d_{\epsilon}}\left(P_{\epsilon}^{\sqcup}\right)
$$




$$
T_{\phi_{f v}, d}(P)=(-1)^{j} T_{\phi_{4}, d_{\beta}}\left(P_{\beta}^{\sqcup}\right)
$$

These diagrams also illustrate the following lemma.

Lemma 75. Consider $c \in D(P)$ such that $T_{\phi_{f v}, c}(P) \quad\left(\right.$ and $T_{\phi_{\sigma f v}, \sigma c}(\sigma P)$ ) only have root and leaf vertexes. Let $\left\{P_{c}^{0}, \ldots P_{c}^{|c|}\right\}$ be the set of labels of $T_{\phi_{f v}, c}(P)$, with $\left\{P_{c}^{0}, \ldots P_{c}^{m}\right\}$ labeling the root vertexes. Then

$$
\Delta_{c} \Lambda_{\phi_{f v}}(P)=\amalg_{j=0}^{m} \Lambda_{*}\left(P_{c}^{j}\right) \otimes \amalg_{i=m+1}^{|c|} \Lambda_{*} P_{c}^{i}
$$

with

$$
*=\left\{\begin{array}{ll}
\phi_{f v} & \text { if }\left(P_{c}^{i}\right) \text { contains first vertex of } P \\
\phi_{4} & \text { else. }
\end{array} .\right.
$$

Similarly,

$$
\Delta_{\sigma c} \Lambda_{\sigma f v}(\sigma P)=\amalg_{j=0}^{m} \Lambda_{*}\left((\sigma P)_{\sigma c}^{j}\right) \otimes \amalg_{i=m+1}^{|c|} \Lambda_{*}\left((\sigma P)_{\sigma c}^{i}\right)
$$

with

$$
*= \begin{cases}\phi_{\sigma f v} & \text { if } P_{c}^{i} \text { contains first vertex of } P \\ \phi_{4} & \text { else. }\end{cases}
$$


Proof. For a fixed $c$ as above and any dissection $d \in D(P)$, such that $c \subset d$, write $d=c \cup_{i=1}^{|c|} d_{i}$ with $d_{i} \in D\left(P_{c}^{i}\right)$. By definition of $\phi_{f v}, d_{i} \cap f v(P)=\emptyset$ if and only if $P_{c}^{i}$ does not inherit the first vertex from $P$. That is, if and only if

$$
T_{\phi_{f v}, d_{i}}\left(P_{c}^{i}\right)=T_{\phi_{4}, d_{i}}\left(P_{c}^{i}\right)
$$

Varying over all dissections $d$ containing $c$ shows that

$$
\Lambda_{\phi_{f v}}\left(P_{c}^{i}\right)=\Lambda_{\phi_{4}}\left(P_{c}^{i}\right)
$$

if and only if $P_{c}^{i}$ does not inherit the first vertex of $P$.

By abuse of notation, call the dissections $c \in D(P)$ such that $T_{\phi_{f v}, c}(P)$ only has leaf and root vertexes the admissible dissections of $P$ in $\phi_{f v}$.

Corollary 76. Using the definitions above, write

$$
\Delta \Lambda_{\phi_{f v}}(P)=\sum_{c \text { admis. dis. }} \Delta_{c} \Lambda_{\phi_{f v}}(P)
$$

and

$$
\Delta \Lambda_{\phi_{\sigma f v}}(\sigma P)=\sum_{c \text { admis. dis. }} \Delta_{c} \Lambda_{\phi_{\sigma f v}}(\sigma P) .
$$

Instead of calculating $\Lambda_{\phi_{4}}(P)-\Lambda_{\phi_{4}}(\sigma P)$, I calculate the expression

$$
\begin{aligned}
& \left.\left.\left.\left.\left(\Lambda_{\phi_{4}}(P)\right)-\Lambda_{\phi_{f v}}(P)\right)\right)-\left(\Lambda_{\phi_{4}}(\sigma P)\right)-\Lambda_{\phi_{\sigma f v}}(\sigma P)\right)\right) \\
& \left.\quad+\left(\Lambda_{\phi_{f v}}(P)\right)-\Lambda_{\phi_{\sigma f v}}(\sigma P)\right) .
\end{aligned}
$$

This is done in steps. The first two terms of (2.9) are derived from Theorem 54; the third term can be calculated up to a primitive coideal.

Lemma 77. Let $P=1, \ldots, n+1$ be an $R$-deco polygon. For $d \subset f v(P)$, let $\left\{P_{d}^{0}, \ldots, P_{d}^{j}\right\}$ be the polygons decorating $T_{\phi_{4}, d}(P)$. There are two expressions for

$$
\begin{gathered}
\left(\Lambda_{\phi_{4}}(P)-\Lambda_{\phi_{f v}}(P)\right)-\left(\Lambda_{\phi_{4}}(\sigma P)-\Lambda_{\phi_{\sigma f v}}(\sigma P)\right) \\
(1)=\sum_{\left((\sigma P)_{\sigma \alpha_{i}}^{r}\right.}^{n} \Lambda_{\phi_{f v}}\left(P_{\alpha_{i}}^{l}\right) \amalg \Lambda_{\phi_{4}}\left(P_{\alpha_{i}}^{r}\right)-\sum_{i=2}^{n} \Lambda_{\phi_{4}}\left((\sigma P)_{\sigma \alpha_{i}}^{l}\right) \amalg \Lambda_{\phi_{\sigma f v}} \\
(2)=-\sum_{d \in f v(P)}(-1)^{|d|} \amalg_{j=0}^{|d|} \Lambda_{\phi_{4}}\left(P_{d}^{j}\right)-\amalg_{j=0}^{|d|} \Lambda_{\phi_{4}}\left(\sigma\left(P_{d}^{j}\right)\right) .
\end{gathered}
$$


Proof. To see expression (1), note that for $\alpha_{i}, \alpha_{j} \in f v(P)$, with $i<j, \alpha_{i}$ dissects the subpolygon $P_{\alpha_{j}}^{r}$, and $\alpha_{j}$ dissects the subpolygon $P_{\alpha_{i}}^{l}$. For $\sigma \alpha_{i}$, $\sigma \alpha_{j} \in \sigma f v(\sigma P)$, with $i<j, \sigma \alpha_{i}$ dissects the subpolygon $(\sigma P)_{\sigma \alpha_{j}}^{r}$, and $\sigma \alpha_{j}$ dissects the subpolygon $(\sigma P)_{\sigma \alpha_{i}}^{l}$. Therefore the generators $T_{\phi_{f v}, f v(P)}(P)$ and $T_{\phi_{\sigma f v}, \sigma f v(P)}(\sigma P)$ are linear, and the result follows form Corollarys 56 and 57.

To see expression (2), Theorem 54 gives

$$
\left(\Lambda_{\phi_{4}}(P)-\Lambda_{\phi_{f v}}(P)\right)=\sum_{\substack{d \subset f v(P) \\ d \neq \emptyset}}(-1)^{|d|+1} \amalg_{j=0}^{|d|} \Lambda_{\phi_{4}}\left(P_{d}^{j}\right)
$$

and

$$
\left(\Lambda_{\phi_{4}}(\sigma P)-\Lambda_{\phi_{\sigma f v}}(\sigma P)\right)=\sum_{\substack{d \subset f v(P) \\ d \neq \emptyset}}(-1)^{|d|+1} \amalg_{j=0}^{|d|} \Lambda_{\phi_{4}}\left(\sigma\left(P_{d}^{j}\right)\right) .
$$

In the following example, I compute $\left(\Lambda_{\phi_{4}}(P)-\Lambda_{\phi_{f v}}(P)\right)$ and $\left(\Lambda_{\phi_{4}}(\sigma P)\right.$ $\left.-\Lambda_{\phi_{\sigma f v}}(\sigma P)\right)$ for a polygon of weight 3 .

Example 78. Consider $P=1234$. By expression (1) of Lemma 77, one has

$$
\begin{aligned}
\Lambda_{\phi_{4}}(P)-\Lambda_{\phi_{f v}}(P)= & \Lambda_{\phi_{4}}(12) \amalg \Lambda_{\phi_{4}}(234)+\Lambda_{\phi_{4}}(123) \amalg \Lambda_{\phi_{4}}(34) \\
& -\Lambda_{\phi_{4}}(12) \amalg \Lambda_{\phi_{4}}(23) \amalg \Lambda_{\phi_{4}}(34) .
\end{aligned}
$$

Applying Theorem 67 gives

$$
\begin{aligned}
q_{2}\left(\Lambda_{\phi_{4}}(P)-\Lambda_{\phi_{f v}}(P)\right)= & q_{2}\left(\Lambda_{\phi_{2}}(12) \amalg\left(\Lambda_{\phi_{2}}(234)+\Lambda_{\phi_{2}}(24) \amalg \Lambda_{\phi_{2}}(32)\right)\right. \\
& +\left(\Lambda_{\phi_{2}}(123)+\Lambda_{\phi_{2}}(13) \amalg \Lambda_{\phi_{2}}(21)\right) \amalg \Lambda_{\phi_{2}}(34) \\
& \left.-\Lambda_{\phi_{2}}(12) \amalg \Lambda_{\phi_{2}}(23) \amalg \Lambda_{\phi_{2}}(34)\right) .
\end{aligned}
$$

The expression involving $\sigma P=2341$ is, using expression (1) of Lemma 77,

$$
\begin{aligned}
\Lambda_{\phi_{4}}(\sigma P)-\Lambda_{\phi_{\sigma f v}}(\sigma P)= & \Lambda_{\phi_{4}}(21) \amalg \Lambda_{\phi_{4}}(342)+\Lambda_{\phi_{4}}(231) \amalg \Lambda_{\phi_{4}}(43) \\
& -\Lambda_{\phi_{4}}(43) \amalg \Lambda_{\phi_{4}}(32) \amalg \Lambda_{\phi_{4}}(21) .
\end{aligned}
$$


Applying Theorem 67 gives

$$
\begin{aligned}
q_{2}\left(\Lambda_{\phi_{4}}(\sigma P)-\Lambda_{\phi_{f v}}(\sigma P)\right)= & q_{2}\left(\Lambda_{\phi_{2}}(21) \amalg\left(\Lambda_{\phi_{2}}(342)+\Lambda_{\phi_{2}}(32) \amalg \Lambda_{\phi_{2}}(43)\right)\right. \\
& +\left(\Lambda_{\phi_{2}}(231)+\Lambda_{\phi_{2}}(21) \amalg \Lambda_{\phi_{2}}(32)\right) \amalg \Lambda_{\phi_{2}}(43) \\
& \left.-\Lambda_{\phi_{2}}(43) \amalg \Lambda_{\phi_{2}}(32) \amalg \Lambda_{\phi_{2}}(21)\right) .
\end{aligned}
$$

Adding the two expressions gives

$$
\begin{aligned}
& q_{2}\left(\Lambda_{\phi_{4}}(P)-\Lambda_{\phi_{f v}}(P)-\Lambda_{\phi_{4}}(\sigma P)+\Lambda_{\phi_{\sigma f v}}(\sigma P)\right) \\
&= q_{2}\left(\Lambda_{\phi_{2}}(12) \amalg \Lambda_{\phi_{2}}(234)+\Lambda_{\phi_{2}}(123) \amalg \Lambda_{\phi_{2}}(34)\right. \\
&-\Lambda_{\phi_{2}}(231) \amalg \Lambda_{\phi_{2}}(43)-\Lambda_{\phi_{2}}(21) \amalg \Lambda_{\phi_{2}}(342) \\
&\left.+\Lambda_{\phi_{2}}(12) \amalg \Lambda_{\phi_{2}}(24) \amalg \Lambda_{\phi_{2}}(32)+\Lambda_{\phi_{2}}(13) \amalg \Lambda_{\phi_{2}}(21)\right) \amalg \Lambda_{\phi_{2}}(34) \\
&\left.-\Lambda_{\phi_{2}}(12) \amalg \Lambda_{\phi_{2}}(23) \amalg \Lambda_{\phi_{2}}(34)\right)-\Lambda_{\phi_{2}}(21) \amalg \Lambda_{\phi_{2}}(32) \amalg \Lambda_{\phi_{2}}(43) .
\end{aligned}
$$

The rest of this section calculates the third term in (2.9).

Definition 79. Let $J_{n} \subset \mathcal{P}_{\bullet}^{(1)}(R)$ be the linear subspace generated by $\{P$ $\sigma P \mid P$ polygon of weight $n\}$. These are primitive coideals in $B_{\phi_{4}}$.

Theorem 80. Define the quotient map

$$
r_{n}: \bar{T}(V(R)) \rightarrow \bar{T}(V(R)) /\left(\sum_{k=1}^{n} J_{k}\right) .
$$

Then for $P$ a polygon of weight $n$,

$$
\left.r_{n}\left(\left(\Lambda_{\phi_{f v}}(P)\right)-\Lambda_{\phi_{\sigma f v}}(\sigma P)\right)\right)=0 .
$$

The coideals $J_{n}$ are problematic in this context since $J_{1}$ is not in the kernel of $\Phi$ (Equation (1.1)),

$$
\Phi([a b]-[b a])=\mathbb{L i}\left(\frac{a}{b}\right)-\mathbb{L i}\left(\frac{b}{a}\right)=\ln (b)-\ln (a),
$$

as shown in Example 71. Instead, I work with a modified quotient map.

Definition 81. Define an ideal $\tilde{J}_{1}=\{a c-c a+c b-b c-a b+b a\}$ to reflect the image of $\Phi$ restricted to 2 -gons. Define the quotient map

$$
\tilde{r}_{n}: \bar{T}(V(R)) \rightarrow \bar{T}(V(R)) /\left(\tilde{J}_{1}+\sum_{k=2}^{n} J_{k}\right) .
$$


I need the following definition to calculate $\tilde{r}_{n}\left(\Lambda_{\phi_{f v}}(P)-\Lambda_{\phi_{\sigma f v}}(P)\right)$.

Definition 82. For $P=1 \ldots n+1$ be polygon of weight $n$, define two polygons $A_{P}=2 \ldots n+1$ and $B_{P}=2 \ldots n 1$ of weight $n-1$. The polygon $A_{P}$ can be drawn as a subpolygon of $P, A_{P}=P_{\alpha_{2}}^{l}$. Define

$$
A_{P, \alpha_{i}}^{l}=P_{\alpha_{i+1}}^{l}, \text { and } A_{P, \alpha_{i}}^{r}=P_{2 \alpha_{i+1}}^{r} \text {. }
$$

Similarly, $B_{P}$ can be drawn as a subpolygon of $\sigma P, B_{P}=(\sigma P)_{\sigma \alpha_{n}}^{r}$. Define

$$
B_{P, \sigma \alpha_{i}}^{l}=\sigma\left(P_{n+1}^{l} \alpha_{i}\right)=(\sigma P)_{\sigma\left({ }_{n+1} \alpha_{i}\right)}^{l} \text {, and } B_{P, \sigma \alpha_{i}}^{r}=\sigma\left(P_{\alpha_{i}}^{r}\right)=(\sigma P)_{\sigma \alpha_{i}}^{r} .
$$

Recall that in the above notation, when I write $P_{\beta}^{r}$, I mean $\beta \in D(P)$. For example, when I write $A_{P, \alpha_{i}}^{l}, \alpha_{i} \in D\left(A_{P}\right)$, whereas in $P_{\alpha_{i+1}}^{l}, \alpha_{i+1} \in D(P)$.

Example 83. The following diagram shows $A_{P, \alpha_{4}}^{l}, A_{P, \alpha_{4}}^{r}, B_{\sigma P, \sigma \alpha_{4}}^{l}$ and $B_{\sigma P, \sigma \alpha_{4}}^{r}$
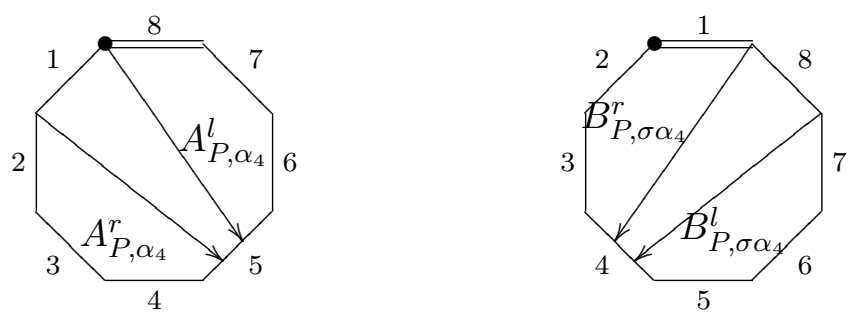

Definition 84. Write $\{\alpha, d, P\}=\left\{v_{r, \alpha}, v_{l, \alpha}\right\}$ to indicate the set of subpolygons to the right and left of the dissecting arrow $\alpha \in d \in D(P)$.

Example 85. For example, $\{\beta, d, P\}$, for the polygons $P=123456$ and dissection $d=\{\alpha, \beta, \gamma\}$ as drawn below,

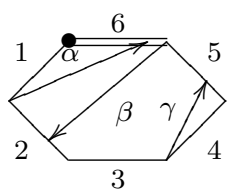

is given by $\{\beta, P\}=\left\{v_{r, \beta}=26, v_{l, \beta}=352\right\}$

I now construct a generalization of the insertion operators $\star_{\prec v}$ and $\star_{\succ v}$, defined in Definition 23 to apply to words of the form $\Lambda_{\phi}(P)$. 
Definition 86. Consider $u \in V(R)$, and $P$ and $R$-deco polygon. Define

$$
u \star \prec\{\alpha, P\} \Lambda_{\phi}(P)=\sum_{\substack{d \in D(P) \\ d=d^{r} \cup d^{l} \cup \alpha}} u \star \prec\{\alpha, d, P\} \Lambda_{\phi}\left(T_{\phi, d^{r}}\left(P_{\alpha}^{r}\right)\right) \amalg \Lambda_{\phi}\left(T_{\phi, d^{l}}\left(P_{\alpha}^{l}\right)\right)
$$

where $d^{r} \in D\left(P_{\alpha}^{r}\right)$ and $d^{l} \in D\left(P_{\alpha}^{l}\right)$. Similarly

$$
u \star_{\succ\{\alpha, P\}} \Lambda_{\phi}(P)=\sum_{\substack{d \in D(P) \\ d=d^{r} \cup d^{l} \cup \alpha}} u \star_{\succ\{\alpha, d, P\}} \Lambda_{\phi}\left(T_{\phi, d^{r}}\left(P_{\alpha}^{r}\right)\right) \amalg \Lambda_{\phi}\left(T_{\phi, d^{l}}\left(P_{\alpha}^{l}\right)\right)
$$

The next theorem gives an expression for $\Lambda_{\phi_{f v}}(P)-\Lambda_{\phi_{\sigma f v}}(\sigma P)$.

Theorem 87. Let $P, A_{P}, B_{P}$ and associated subpolygons be as above. Define the quotient map

$$
\tilde{r}_{n}: \Lambda\left(\mathfrak{T}_{\phi_{4}}\right) \rightarrow \Lambda\left(\mathfrak{T}_{\phi_{4}}\right) /\left(\tilde{J}_{1}+\sum_{k=2}^{n} J_{k}\right)
$$

For $P$ an $R$-deco polygon of weight $n \geq 2$,

$$
\begin{aligned}
\left.\tilde{r}_{n}\left(\Lambda_{\phi_{f v}}(P)-\Lambda_{\phi_{\sigma f v}}(\sigma P)\right)\right) \\
=\sum_{i=2}^{n} \tilde{r}_{n}\left((i(n+1)-(n+1) i) \star \prec\left\{\alpha_{i-1}, A_{P}\right\} \Lambda_{\phi_{4}}\left(A_{P, \alpha_{i-1}}^{r}\right) \amalg \Lambda_{\phi_{f v}}\left(A_{P, \alpha_{i-1}}^{l}\right)\right. \\
\quad-(i 1-1 i) \star \succ\left\{\sigma \alpha_{i}, B_{P}\right\} \\
\left.\quad \Lambda_{\phi_{4}}\left(B_{P, \sigma \alpha_{i}}^{l}\right) \amalg \Lambda_{\phi_{\sigma f v}}\left(B_{P, \sigma \alpha_{i}}^{r}\right)\right) .
\end{aligned}
$$

Remark 88. Note that

$$
\begin{aligned}
i(n+1)-(n+1) i \star \prec\left\{\alpha_{i-1}, A_{P}\right\} & \left(\Lambda_{\phi_{4}}\left(A_{P, \alpha_{i-1}}^{r}\right) \amalg \Lambda_{\phi_{f v}}\left(A_{P, \alpha_{i-1}}^{l}\right)\right) \\
= & i(n+1) \star_{\prec\left\{\alpha_{i-1}, A_{P}\right\}}\left(\Lambda_{\phi_{4}}\left(A_{P, \alpha_{i-1}}^{r}\right) \amalg \Lambda_{\phi_{f v}}\left(A_{P, \alpha_{i-1}}^{l}\right)\right) \\
& -(n+1) i \star \prec\left\{\alpha_{i-1}, A_{P}\right\} \\
& \left(\Lambda_{\phi_{4}}\left(A_{P, \alpha_{i-1}}^{r}\right) \amalg \Lambda_{\phi_{f v}}\left(A_{P, \alpha_{i-1}}^{l}\right)\right) .
\end{aligned}
$$

Before proving this theorem, I use it to prove Theorem 80.

Proof of Theorem 80. Since every term in Theorem 26 calculation involves a term in $J_{1}$, specifically, the newly inserted difference,

$$
r_{1} \circ \tilde{r}_{n}\left(\Lambda_{f v}(P)-\Lambda_{\sigma f v}(\sigma P)\right)=0
$$

for any $R$-deco polygon $P$. 
I have now computed all the necessary terms for $\Lambda_{\phi_{4}}(P)-\Lambda_{\phi_{4}}(\sigma P)$. Let $P$ be a polygon of weight $n$. Combining line (2) of Lemma 77 with Theorem 87 gives

$$
\begin{aligned}
\tilde{r}_{n}\left(\Lambda_{\phi_{4}}(P)-\Lambda_{\phi_{4}}(\sigma P)\right) & -\sum_{d \in f v(P)}(-1)^{|d|} \amalg_{j=0}^{|d|}\left(\Lambda_{\phi_{4}}\left(P_{d}^{j}\right)-\amalg_{j=0}^{|d|} \Lambda_{\phi_{4}}\left(\sigma\left(P_{d}^{j}\right)\right)\right) \\
& +\tilde{r}_{n} \sum_{i=2}^{n}\left(i(n+1)-(n+1) i \star \prec\left\{\alpha_{i-1}, A_{P}\right\} \Lambda_{\phi_{4}}\left(A_{P, \alpha_{i-1}}^{r}\right) \amalg \Lambda_{\phi_{f v}}\left(A_{P, \alpha_{i-1}}^{l}\right)\right) \\
& \quad-(i 1-1 i) \star \succ\left\{\sigma \alpha_{i}, B_{P}\right\} \\
& \left.\quad \Lambda_{\phi_{4}}\left(B_{P, \sigma \alpha_{i}}^{l}\right) \amalg \Lambda_{\phi_{\sigma f v}}\left(B_{P, \sigma \alpha_{i}}^{r}\right)\right),
\end{aligned}
$$

where $\left\{P_{d}^{0}, \ldots, P_{d}^{|d|}\right\}$ decorate the vertexes of $T_{\phi_{4}, d}(P)$, for $d \subset f v(P)$.

Proof of Theorem 8\%. Let $c_{r}$ be an admissible dissection of $A_{P, \alpha_{i}-1}^{r}$ in $\Lambda_{\phi_{4}}$ and $c_{l}$ of $A_{P, \alpha_{i}-1}^{l}$ in $\Lambda_{\phi_{f v}}$. By Definition 86 and Lemma 25,

$$
\begin{aligned}
& \Delta(i(n+1)-(n+1) i) \star \prec\left\{\alpha_{i-1}, A_{P}\right\}\left(\Lambda_{\phi_{4}}\left(A_{P, \alpha_{i-1}}^{l}\right) \amalg \Lambda_{\phi_{f v}}\left(A_{P, \alpha_{i-1}}^{r}\right)\right) \\
& =\sum_{c_{r}, c_{l}} \Lambda_{\phi_{4}}\left(R_{c_{r}}\left(A_{P, \alpha_{i-1}}^{r}\right)\right) \amalg \Lambda_{\phi_{f v}}\left(R_{c_{l}}\left(A_{P, \alpha_{i-1}}^{l}\right)\right) \otimes(i(n+1) \\
& -(n+1) i) \star_{\prec\left\{\alpha_{i-1}, A_{P}\right\}}\left(\Lambda_{\phi_{4}}\left(L_{c_{r}}\left(A_{P, \alpha_{i-1}}^{r}\right)\right) \amalg \Lambda_{\phi_{f v}}\left(L_{c_{l}}\left(A_{P, \alpha_{i-1}}^{l}\right)\right)\right) \\
& +\sum_{c_{r}, c_{l}}(i(n+1)-(n+1) i) \star \prec\left\{\alpha_{i-1}, A_{P}\right\}\left(\Lambda_{\phi_{4}}\left(R_{c_{r}}\left(A_{P, \alpha_{i-1}}^{r}\right)\right) \amalg\right. \\
& \left.\Lambda_{\phi_{f v}}\left(R_{c_{l}}\left(A_{P, \alpha_{i-1}}^{l}\right)\right)\right) \otimes \Lambda_{\phi_{4}}\left(L_{c_{r}}\left(A_{P, \alpha_{i-1}}^{r}\right)\right) \amalg \Lambda_{\phi_{f v}}\left(L_{c_{l}}\left(A_{P, \alpha_{i-1}}^{l}\right)\right) \\
& +\sum_{c_{r}, c_{l}}(i(n+1)-(n+1) i) \star \prec v_{r, \alpha_{i-1}}\left(\Lambda_{\phi_{4}}\left(R_{c_{r}}\left(A_{P, \alpha_{i-1}}^{r}\right)\right) \amalg\right. \\
& \left.\Lambda_{\phi_{f v}}\left(R_{c_{l}}\left(A_{P, \alpha_{i-1}}^{l}\right)\right)\right) \otimes \Lambda_{\phi_{4}}\left(L_{c_{r}}\left(A_{P, \alpha_{i-1}}^{r}\right)\right) \amalg \Lambda_{\phi_{f v}}\left(L_{c_{l}}\left(A_{P, \alpha_{i-1}}^{l}\right)\right) \\
& +\sum_{c_{r}, c_{l}} \Lambda_{\phi_{4}}\left(R_{c_{r}}\left(A_{P, \alpha_{i-1}}^{r}\right)\right) \amalg(i(n+1)-(n+1) i) \star \prec v_{l, \alpha_{i-1}} \\
& \Lambda_{\phi_{f v}}\left(R_{c_{l}}\left(A_{P, \alpha_{i-1}}^{l}\right)\right) \otimes \Lambda_{\phi_{4}}\left(L_{c_{r}}\left(A_{P, \alpha_{i-1}}^{r}\right)\right) \amalg \Lambda_{\phi_{f v}}\left(L_{c_{l}}\left(A_{P, \alpha_{i-1}}^{l}\right)\right) .
\end{aligned}
$$

There is a similar expression for

$$
\Delta\left((i 1-1 i) \star_{\succ\left\{\sigma \alpha_{i}, B_{P}\right\}} \Lambda_{\phi_{\sigma f v}}\left(B_{P, \alpha_{i}}^{l}\right) \amalg \Lambda_{\phi_{4}}\left(B_{P, \alpha_{i}}^{r}\right)\right) .
$$


This proof proceeds by comparing the coproduct of both sides of (2.11). In fact, I only consider the coproduct of terms involving the inserted sum $i(n+1)-(n+1) i$. The arguments for $i 1-1 i$ are similar, and not done here.

This proof proceeds by induction. Note from Example 71 that this theorem holds for $n=2$, with $P=123$. By expression (1) of Lemma 77

$$
\left(\Lambda_{\phi_{4}}(P)-\Lambda_{\phi_{f v}}(P)\right)-\left(\Lambda_{\phi_{4}}(\sigma P)-\Lambda_{\phi_{\sigma f v}}(\sigma P)\right)=23 \amalg 12-21 \amalg 32 .
$$

Comparing this to expression (2.8) in Example 71 gives

$$
\begin{aligned}
& \Lambda_{\phi_{f v}}(P)-\Lambda_{\phi_{\sigma f v}}(\sigma P) \\
& \quad=[123]-[231]+[13-31-12+21 \mid 23]+[21 \mid 13-31-23+32] .
\end{aligned}
$$

In this case, the polygons $A=23$, and $B=21$. Recall that modulo $\tilde{J}_{1}$, $13-31-12+21=23-32$ and $13-31-23+32=12-21$. Therefore, under the quotient map $\tilde{r}_{n}$,

$$
\Lambda_{\phi_{f v}}(P)-\Lambda_{\phi_{\sigma f v}}(\sigma P)=[23-32 \mid 23]-[21 \mid 21-12]
$$

as desired. Suppose Equation (2.11) holds for all polygons of weight $m$ for $m<n$.

Consider a general polygon $P$ of weight $n$. Let $c$ be an admissible dissection of $P$ in $\phi_{f v}$ as in Corollary 76. Since the generator $T_{\phi_{f v}, f v(P)}(P)$ is linear, $c$ contains at most one arrow in $f v(P)$. Therefore, there are two cases to consider: $c \cap f v(P)=\emptyset$, and $|c \cap f v(P)|=1$.

If $c \cap f v(P)=\emptyset$, there is only one label of $T_{\phi_{f v}, c}(P)$ which inherits its first vertex from $P, P_{c}^{\bullet}$. By Lemma 75 , the generators $T_{\phi_{f v}, c}(P)$ and $T_{\phi_{\sigma f v}, \sigma c}(\sigma P)$ are identical after replacing $P_{c}^{\bullet}$ with $\sigma P_{c}^{\bullet}$. The dissection $c$ contains either an arrow ending on the root side, $\gamma_{c}(n+1)$, or one ending on the first side $\gamma_{c}(1)$, but not both. If $P_{c}^{\bullet}$ is a root (resp. leaf) label of $T_{f v, c}(P), c$ contains $\gamma_{c}(n+1)$ (resp. $\gamma_{c}(1)$ ). Either arrow may be trivial. Write $c^{\prime}=c \backslash\left\{\gamma_{c}(n+1), \gamma_{c}(1)\right\}$ and

$$
P_{c^{\prime}}^{\bullet}=1 a_{2} \cdots a_{l} n+1
$$

the polygon associated to the dissection $c^{\prime}$ that inherits the first vertex of $P$. Write $a_{1}=1, c_{1}=c^{\prime} \cup \gamma_{c}\left(a_{1}\right), a_{l+1}=n+1, c_{l+1}=c^{\prime} \cup \gamma_{c}\left(a_{l+1}\right)$. Define polygons $Q_{i}$ and $T_{i}$ such that $Q_{i}=\left(P_{c^{\prime}}^{\bullet-}\right)_{\gamma_{c}\left(a_{i}\right)}^{l}$, and $T_{i}=\left(P_{c^{\prime}}^{\bullet-}\right)_{\gamma_{c}\left(a_{i}\right)}^{r}$ for 
$i \in\{1, l+1\}$. Write the polygon

$$
Q_{l+1}=1 a_{2} \cdots a_{q}(n+1) .
$$

for later use, define a family of arrows

$$
\gamma_{c}\left(a_{m}\right)={ }_{a_{q}+1} \alpha_{a_{m}}
$$

By construction $P_{c}^{\bullet} \in\left\{Q_{i}, T_{i}\right\}$. If $\gamma\left(a_{l+1}\right)$ is trivial, $a_{q}=n$ and $P_{c}^{\bullet}=Q_{l+1}$ decorates a root vertex of $T_{\phi_{f v}, c}(P)$, and $T_{l+1}$ a trivial polygon. If $\gamma\left(a_{1}\right)$ is trivial, $a_{1}=1$ and $P_{c}^{\bullet}=T_{1}$ is a leaf polygon of $T_{\phi_{f v}, c}(P)$, and $Q_{1}$ a trivial polygon.

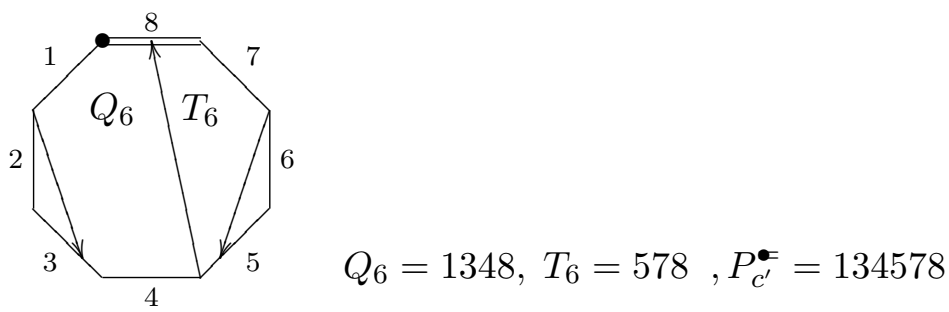

Let $\left\{R_{1}, \ldots, R_{l}, Q_{i}, T_{i}, L_{1}, \ldots, L_{n}\right\}$ be the labels of $T_{f v, c_{i}}(P)$ for $i \in$ $\{1, l+1\}$, with the $R_{k}$ and $L_{j}$ corresponding to root and leaf labels respectively. By Lemma 75 , I write

$$
\begin{aligned}
\Delta_{c_{1}} & +\Delta_{c_{l+1}}\left(\Lambda_{\phi_{f v}}(P)-\Lambda_{\phi_{\sigma f v}}(\sigma P)\right) \\
= & \amalg_{k} \Lambda_{\phi_{4}}\left(R_{k}\right) \amalg \Lambda_{\phi_{f v}}\left(Q_{1}\right) \otimes\left(\Lambda_{\phi_{f v}}\left(T_{1}\right)-\Lambda_{\phi_{\sigma f v}}\left(\sigma T_{1}\right)\right) \amalg_{j} \Lambda_{\phi_{4}}\left(L_{j}\right) \\
& +\left(\Lambda_{\phi_{f v}}\left(Q_{l+1}\right)-\Lambda_{\phi_{\sigma f v}}\left(\sigma Q_{l+1}\right)\right) \amalg_{k} \Lambda_{\phi_{4}}\left(R_{k}\right) \otimes \amalg_{j} \Lambda_{\phi_{4}}\left(L_{j}\right) \\
& \quad \amalg \Lambda_{\phi_{\sigma f v}}\left(\sigma T_{l+1}\right) .
\end{aligned}
$$

Consider the case of admissible cuts such that $c \cap f v(P)=1$. Specifically, consider admissible dissections of $P$ in $\Lambda_{f v}(P)$ of the form $c_{m}=c^{\prime} \cup$ $\alpha_{a_{m}}, \gamma_{c}\left(a_{m}\right)$ for $m \in\{2 \ldots l\}$ (where $\gamma_{c}\left(a_{m}\right)$ defined as in Equation (2.17)). For $d \supseteq\left\{\alpha_{a_{m}}, \gamma_{c}\left(a_{m}\right)\right\}$, let the polygons $Q_{m}, T_{m}$ and $S_{m}$ decorate the vertexes of $T_{\phi_{f v}, d}\left(P_{c^{\prime}}^{\bullet}\right)$ such that $Q_{m} \prec T_{m}$ in $T_{\phi_{f v}, d}(P)$ are adjacent to the arrow $\alpha_{a_{m}}$ and $Q_{m} \prec S_{m}$, with both adjacent to the arrow $\gamma_{c}\left(\alpha_{m}\right)$. The polygon $S_{m}$ decorates the remaining vertex. If $m=q+1$ (or $q$ ), then $S_{m}$ is trivial, and $T_{m}=T_{l+1}\left(\right.$ or $\left.Q_{m}=Q_{1}\right)$. 

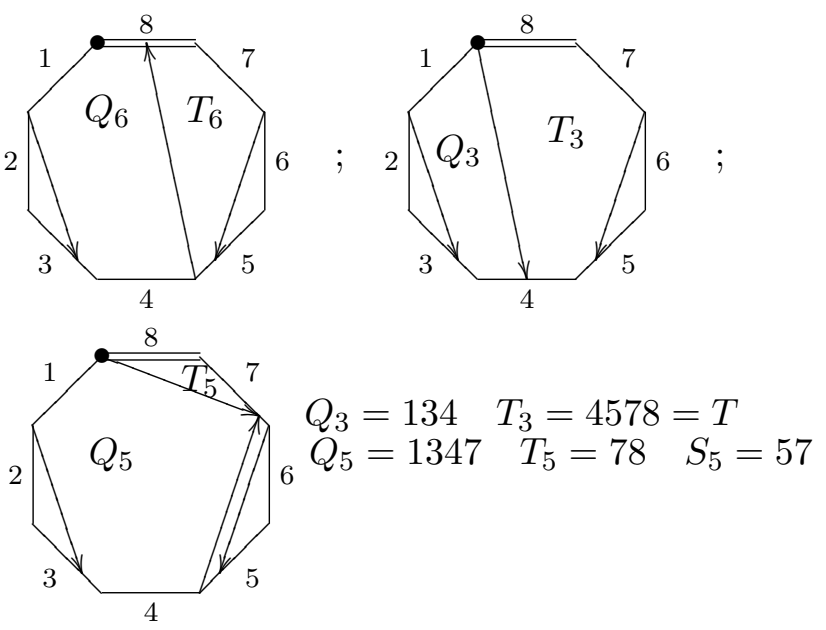

Write

$$
\begin{aligned}
& \left.\sum_{m=2}^{l} \Delta_{c_{m}}\left(\Lambda_{\phi_{f v}}(P)\right)-\Lambda_{\phi_{\sigma f v}}(\sigma P)\right) \\
& \quad=-\sum_{m=2}^{l} \amalg_{k} \Lambda_{\phi_{4}}\left(R_{k}\right) \amalg \Lambda_{\phi_{f v}}\left(Q_{m}\right) \otimes\left(\Lambda_{\phi_{f v}}\left(T_{m}\right)-\Lambda_{\phi_{\sigma f v}}\left(\sigma T_{m}\right)\right) \\
& \quad \amalg_{j} \Lambda_{\phi_{4}}\left(L_{j}\right)+\amalg_{k} \Lambda_{\phi_{4}}\left(R_{k}\right) \amalg\left(\Lambda_{\phi_{f v}}\left(Q_{m}\right)-\Lambda_{\phi_{\sigma f v}}\left(\sigma Q_{m}\right)\right) \\
& \quad \otimes \Lambda_{\phi_{\sigma f v}}\left(\sigma T_{m}\right) \amalg_{j} \Lambda_{\phi_{4}}\left(L_{j}\right)
\end{aligned}
$$

where the negative sign comes from $\operatorname{sign}_{f v}\left(c_{m}\right)$ and the fact that $c_{m} \cap$ $f v(P)=\alpha_{m}$. In this expression, I have included the term $\Lambda_{\phi_{4}}\left(S_{m}\right)$ in the set $\left\{\Lambda_{\phi_{4}}\left(R_{k}\right)\right\}$ if $m \leq q$ and in the set $\left\{\Lambda_{\phi_{4}}\left(L_{k}\right)\right\}$ if $m>q$.

The set of non-trivial admissible dissections of $P$ in $\phi_{f v}$ can be partitioned into sets of the form $\left\{c_{i}\right\}_{i=1}^{l+1}$. For the remainder of the proof, I calculate the contribution to the coproduct from $\sum_{m=1}^{l+1} \Delta_{c_{i}}$. The result can be derived by summing over all such subsets.

From expression (1) of Lemma 77,

$$
\Lambda_{\phi_{4}}\left(T_{l+1}\right)-\Lambda_{\phi_{\sigma f v}}\left(T_{l+1}\right)=\sum_{m=q+2}^{l} \Lambda_{\phi_{\sigma f v}}\left(T_{m}\right) \amalg \Lambda_{\phi_{4}}\left(S_{m}\right) .
$$

Since $T_{q+1}=T_{l+1}$, and $S_{q+1}$ is trivial,

$$
\Lambda_{\phi_{4}}\left(T_{l+1}\right)=\sum_{m=q+1}^{l} \Lambda_{\phi_{\sigma f v}}\left(T_{m}\right) \amalg \Lambda_{\phi_{4}}\left(S_{m}\right) .
$$


Similarly,

$$
\Lambda_{\phi_{4}}\left(Q_{1}\right)=\sum_{m=2}^{q} \Lambda_{\phi_{f v}}\left(Q_{m}\right) \amalg \Lambda_{\phi_{4}}\left(S_{m}\right) .
$$

Inserting Equations (2.20) and (2.21) into (2.18) gives

$$
\begin{aligned}
& \left(\Delta_{c_{1}}+\Delta_{c_{l+1}}\right)\left(\Lambda_{\phi_{f v}}(P)-\Lambda_{\phi_{\sigma f v}}(\sigma P)\right) \\
& =\amalg_{k} \Lambda_{\phi_{4}}\left(R_{k}\right) \amalg\left(\Lambda_{\phi_{f v}}\left(Q_{l+1}\right)-\Lambda_{\phi_{\sigma f v}}\left(\sigma Q_{l+1}\right)\right) \\
& \quad \otimes \sum_{m=q+1}^{l}\left[\Lambda_{\phi_{\sigma f v}}\left(\sigma T_{m}\right) \amalg \Lambda_{\phi_{4}}\left(S_{m}\right)\right] \amalg \Lambda_{j} \Lambda_{\phi_{4}}\left(L_{j}\right) \\
& \quad+\amalg_{k} \Lambda_{\phi_{4}}\left(R_{k}\right) \amalg \sum_{m=2}^{q}\left[\Lambda_{\phi_{f v}}\left(Q_{m}\right) \amalg \Lambda_{\phi_{4}}\left(S_{m}\right)\right] \\
& \quad \otimes\left(\Lambda_{\phi_{f v}}\left(T_{1}\right)-\Lambda_{\phi_{\sigma f v}}\left(\sigma T_{1}\right)\right) \amalg_{j} \Lambda_{\phi_{4}}\left(L_{j}\right)
\end{aligned}
$$

Combining (2.22) with (2.19) gives an expression for

$$
\begin{aligned}
\sum_{m=1}^{l+1} & \Delta_{c_{m}}\left(\Lambda_{\phi_{f v}}(P)-\Lambda_{\phi_{\sigma f v}}(\sigma P)\right) \\
= & \sum_{m=q+1}^{l}\left(\Lambda_{\phi_{f v}}\left(Q_{l+1}\right)-\Lambda_{\phi_{\sigma f v}}\left(\sigma Q_{l+1}\right)-\Lambda_{\phi_{f v}}\left(Q_{m}\right)+\Lambda_{\phi_{\sigma f v}}\left(\sigma Q_{m}\right)\right) \\
& \amalg_{k} \Lambda_{\phi_{4}}\left(R_{k}\right) \otimes\left[\Lambda_{\phi_{\sigma f v}}\left(T_{m}\right) \amalg \Lambda_{\phi_{4}}\left(S_{m}\right)\right] \amalg_{j} \Lambda_{\phi_{4}}\left(L_{j}\right) \\
& -\sum_{m=2}^{q} \amalg_{i} \Lambda_{\phi_{4}}\left(R_{i}\right) \amalg\left(\Lambda_{\phi_{f v}}\left(Q_{m}\right)-\Lambda_{\phi_{\sigma f v}}\left(\sigma Q_{m}\right)\right) \amalg \Lambda_{\phi_{4}}\left(S_{m}\right) \\
& \left.\otimes \Lambda_{\phi_{\sigma f v}}\left(T_{m}\right) \amalg_{j} \Lambda_{\phi_{4}}\left(L_{j}\right)\right) \\
& +\sum_{m=2}^{q} \amalg_{k} \Lambda_{\phi_{4}}\left(R_{k}\right) \amalg\left[\Lambda_{\phi_{f v}}\left(Q_{m}\right) \amalg \Lambda_{\phi_{4}}\left(S_{m}\right)\right] \\
& \otimes\left(\Lambda_{\phi_{f v}}\left(T_{1}\right)-\Lambda_{\phi_{\sigma f v}}\left(\sigma T_{1}\right)-\Lambda_{\phi_{f v}}\left(T_{m}\right)+\Lambda_{\phi_{\sigma f v}}\left(\sigma T_{m}\right)\right) \amalg_{j} \Lambda_{\phi_{4}}\left(L_{j}\right) \\
& -\sum_{m=q+1}^{l} \amalg_{i} \Lambda_{\phi_{4}}\left(R_{i}\right) \amalg \Lambda_{\phi_{f v}}\left(Q_{m}\right) \\
& \otimes \Lambda_{\phi_{4}}\left(S_{m}\right) \amalg\left(\Lambda_{\phi_{f v}}\left(T_{m}\right)-\Lambda_{\phi_{\sigma f v}}\left(\sigma T_{m}\right)\right) \amalg_{j} \Lambda_{\phi_{4}}\left(L_{j}\right)
\end{aligned}
$$


Consider the sum in (2.23). The following arguments are similar for the terms in 2.24. By induction,

$$
\begin{aligned}
\tilde{r}_{n} & \sum_{m=q+1}^{l}\left(\Lambda_{\phi_{f v}}\left(Q_{l+1}\right)-\Lambda_{\phi}\left(\sigma Q_{l+1}\right)-\Lambda_{\phi_{f v}}\left(Q_{m}\right)+\Lambda_{\phi}\left(\sigma Q_{m}\right)\right) \\
= & \tilde{r}_{n} \sum_{m=q+1}^{l} \sum_{i=2}^{q}\left(a_{i}(n+1)-(n+1) a_{i}\right) \star \prec\left\{\alpha_{i-1}, A_{Q}\right\} \\
& \amalg \Lambda_{\phi_{4}}\left(A_{Q_{m}, \alpha_{i-1}}^{r}\right) \\
& \left.\amalg \Lambda_{\phi_{f v}}\left(A_{Q_{m}, \alpha_{i-1}}^{l}\right)\right)-\left(a_{i} a_{m}-a_{m} a_{i}\right) \star \prec\left\{\alpha_{i-1}, A_{Q_{m}}\right\} \\
& \left(\Lambda_{\phi_{4}}\left(A_{Q_{m}, \alpha_{i-1}}^{r}\right) \amalg \Lambda_{\phi_{f v}}\left(A_{Q_{m}, \alpha_{i-1}}^{l}\right)\right) .
\end{aligned}
$$

Since

$$
B_{Q}=B_{Q_{m}}=a_{2} \cdots a_{q} 1
$$

for all $j$, all terms involving $a_{i} 1-a_{i} 1$ cancel. Similarly

$$
\begin{aligned}
\tilde{r}_{n} \sum_{m=2}^{q}\left(\Lambda_{\phi_{f v}}\left(Q_{m}\right)-\Lambda_{\phi}\left(\sigma Q_{m}\right)\right) \\
=\tilde{r}_{n}\left(\sum_{m=2}^{q} \sum_{i=2}^{m-1}\left(a_{i} a_{m}-a_{m} a_{i}\right) \star \prec\left\{\alpha_{i-1}, A_{Q_{m}}\right\}\right. \\
\quad\left(\Lambda_{\phi_{4}}\left(A_{Q_{m}, \alpha_{i-1}}^{r}\right) \amalg \Lambda_{\phi_{f v}}\left(A_{Q_{m}, \alpha_{i-1}}^{l}\right)\right) \\
\quad-\left(a_{i} 1-1 a_{i}\right) \star \prec\left\{\sigma \alpha_{i}, B_{Q_{m}}\right\} \\
\left.\quad\left(\Lambda_{\phi_{4}}\left(B_{Q_{m}, \sigma \alpha_{i}}^{l}\right) \amalg \Lambda_{\phi}\left(B_{Q_{m}, \sigma \alpha_{i}}^{r}\right)\right)\right) .
\end{aligned}
$$

Use the coideal $\tilde{J}_{1}$ to rewrite

$$
a_{i} a_{m}-a_{m} a_{i}=a_{i}(n+1)-(n+1) a_{i}-a_{m}(n+1)+(n+1) a_{m} .
$$

Inserting this into expressions (2.25) and (2.26) and substituting into (2.23) gives an expression for $\sum_{m=2}^{l+1} \Delta_{c_{m}} \Lambda_{\phi_{f v}}(P)-\Lambda_{\sigma f v}(\sigma P)$

$$
\begin{aligned}
& \sum_{i=2}^{q}\left(a_{i}(n+1)-(n+1) a_{i}\right) \star \prec\left\{\alpha_{i-1}, A_{Q_{l+1}}\right\} \\
& \quad \amalg_{k}\left(\Lambda_{\phi_{4}} R_{k}\right) \otimes \Lambda_{\phi_{4}}\left(T_{l+1}\right){A_{Q_{l+1}, \alpha_{i-1}}}_{j}\left(\Lambda_{\phi_{4}} L_{j}\right)
\end{aligned}
$$


$(2.28)$

$$
\begin{aligned}
& +\sum_{m=2}^{q} \sum_{i=2}^{m-1}\left(a_{i} 1-1 a_{i}\right) \star \succ\left\{\alpha_{i}, B_{Q_{m}}\right\} \\
& \left.\amalg_{k}\left(\Lambda_{\phi_{4}} R_{k}\right) \otimes \Lambda_{\phi}\left(T_{m}\right) \amalg_{j} \Lambda_{\phi_{4}}\left(L_{j}\right)\right) \\
& .29) \\
& -\sum_{Q_{m}, \alpha_{i}}^{l} \sum_{i=2}^{l} \sum_{\Lambda_{\phi}}^{\min (m-1, q)}\left(a_{i}(n+1)-(n+1) a_{i}\right) \star \prec\left\{\alpha_{i-1}, A_{Q_{m}}\right\} \\
& \left.\amalg \Lambda_{\phi_{f v}}\left(A_{Q_{m}, \alpha_{i-1}}^{l}\right)\right) \amalg_{\phi_{4}}\left(A_{Q_{m}, \alpha_{i-1}}^{r}\right)
\end{aligned}
$$

$$
\begin{aligned}
& +\sum_{m=2}^{l}\left(a_{m}(n+1)-(n+1) a_{m}\right) \star \prec A_{Q_{m}}^{\star} \Lambda_{\phi_{4}}\left(A_{Q_{m}}\right) \\
& \left.\amalg_{i}\left(\Lambda_{\phi_{4}} R_{i}\right) \otimes \Lambda_{\phi}\left(T_{m}\right) \amalg_{j} \Lambda_{\phi_{4}}\left(L_{j}\right)\right) .
\end{aligned}
$$

Expression for (2.27) is derived with the aid of Equation (2.20). Line (2.28) is derived from line (2.26). In lines (2.28), (2.29) and (2.30) the $S_{m}$ terms are absorbed in to the set of $R_{i}$ (if $m \leq q$ ) or $L_{i}$ (if $m>q$ ). Finally, line (2.30) is derived from statement (1) of Lemma 77, namely that

$$
\Lambda_{\phi_{4}}\left(A_{Q_{m}}\right)=\sum_{i=2}^{\text {weight }}\left(\Lambda_{\phi_{4}}\left(A_{Q_{m}, \alpha_{i-1}}^{r}\right) \amalg \Lambda_{\phi_{f v}}\left(A_{Q_{m}, \alpha_{i-1}}^{l}\right)\right) .
$$

I use this lines $(2.27),(2.28),(2.29)$ and (2.30) to compare (2.23) to the coproduct of the terms involving $i(n+1)-(n+1) i$ in Equation (2.11). Line (2.28) I ignore, as it contributes to the coproduct of terms involving $a_{i} 1-1 a_{i}$. Instead, I consider

$$
\begin{aligned}
- & \left.\sum_{m=q+1}^{l} \sum_{i=m+1}^{l} \amalg_{i}\left(\Lambda_{\phi_{4}} R_{i}\right) \amalg \Lambda_{\phi}\left(Q_{m}\right) \otimes \amalg_{j} \Lambda_{\phi_{4}}\left(L_{j}\right)\right) \amalg \\
& \left(a_{i}(n+1)-a_{i}(n+1)\right) \star \prec\left\{\alpha_{i-1}, A_{T_{m}}\right\} \\
& \left(\Lambda_{\phi_{4}}\left(A_{T_{m}, \alpha_{i-1}}^{l}\right) \amalg \Lambda_{\phi_{f v}}\left(A_{T_{m}, \alpha_{i-1}}^{r}\right)\right)
\end{aligned}
$$

which comes from expression (2.24).

Note that $\left\{c_{m} \mid 1 \leq m \leq l+1\right\}$ are admissible dissections of $P$ in $\phi_{f v}$. They need not be admissible dissections of $A_{P}$ or $B_{P}$ in either $\phi_{4}$ of $\phi_{f v}$. However, each can be partitioned into admissible dissections of the subpolygons $A_{P, \alpha_{i-1}}^{l}$ and $A_{P, \alpha_{i-1}}^{r}$. Line (2.27) corresponds to the admissible cut 
$c_{l+1}$. This can be partitioned $c_{l+1}=c_{A_{P, \alpha_{a}-1}^{r}} \cup c_{A_{P, \alpha_{a_{i}-1}}^{l}}$ for $2 \leq i \leq q$, where $c_{A_{P, \alpha_{a_{i}-1}}^{r}}$ is an admissible dissection of $A_{P, \alpha_{a_{i}-1}}^{l}$ in $\Lambda_{\phi_{f v}}$ and $c_{A_{P, \alpha_{a_{i}-1}}^{r}}$ is an admissible dissection of $A_{P, \alpha_{i-1}}^{r}$ in $\Lambda_{\phi_{4}}$. It cannot be partitioned admissible dissections of $B_{P, \alpha_{a_{i}}}^{r}$ and $B_{P, \alpha_{a_{i}}}^{l}$ for any $i$, as the arrow $\alpha_{q}+1 \alpha \in c$ is not in $D\left(B_{P}\right)$. For instance, for $i=3$,

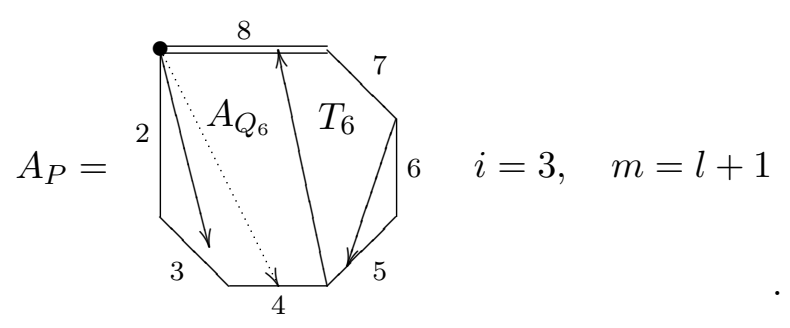

The terms in (2.27) correspond to terms in (2.14) with $c_{r}=c_{A_{P, \alpha_{a_{i}-1}}^{r}}$ and $c_{l}=c_{A_{P, \alpha_{a}-1}^{l}}$.

Consider the admissible dissections contributing to (2.31). Here, $i>m>$ $q$; therefore $\alpha_{a_{m}} \in D\left(B_{P, \alpha_{a_{i}}}^{r}\right)$.

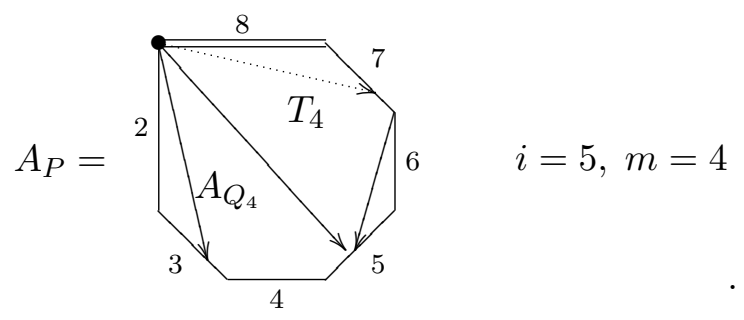

The terms of (2.31) correspond to terms in (2.13), with $c_{r}=c_{A_{P, \alpha_{a_{i}}}^{r}}$ and $c_{l}=c_{A_{P, \alpha_{i}}^{l}} ; c_{m}=c_{A_{P, \alpha_{a_{i}}}^{r}} \cup c_{A_{P, \alpha_{i}}^{l}}$.

For $2 \leq m \leq l$, and $2<i \stackrel{\alpha_{i}}{<}$ wt $Q_{m}$

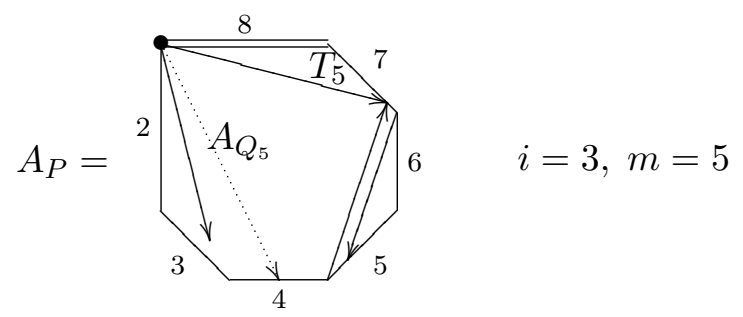

the terms of (2.29) corresponds to terms of (2.14), with $c_{r}=c_{A_{P, \alpha_{i-1}}^{r}}$ and $c_{l}=c_{A_{P, \alpha_{i}-1}^{l}} \cup \alpha_{a_{m}}$. For $i=2$ the subpolygon $\left(A_{Q_{m}}\right)_{\alpha_{a_{2}-1}}^{r}$ is trivial. The 
admissible dissection can be written $c_{m}=c_{A_{P, \alpha_{i-1}}^{r}} \cup c_{A_{P, \alpha_{i}-1}^{l}} \cup \alpha_{a_{2}}$. In this case, the terms of (2.29) corresponds to terms of (2.15), with $c_{r}=c_{A_{P, \alpha_{a_{i}-1}}^{r}}$ and $c_{l}=c_{A_{P, \alpha_{a}-1}^{l}}$.

Finally, the terms of (2.30) corresponds to terms of (2.15), with $c_{r}=c_{A_{P, \alpha_{a_{m}-1}}^{r}}$ and $c_{l}=c_{A_{P, \alpha_{a_{m}-1}}^{l}}$.

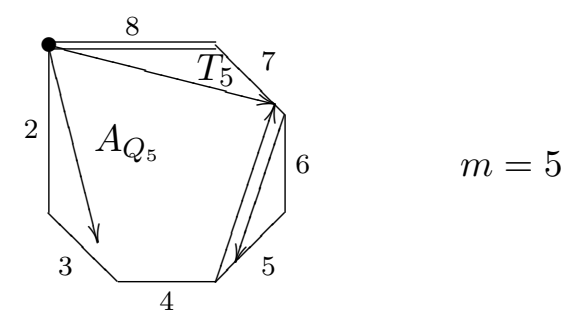

Varying the dissections $c$ and associated $c_{m}$ account for all terms in the expressions $(2.14),(2.13),(2.15)$ and $(2.16)$, showing that the coproduct of the two sides of (2.11) are equal.

\section{Acknowledgments}

The author would like to thank Herbert Gangl for significant guidance and inspiration during the writing of this paper.

\section{References}

[1] S. Bloch and I. Kriz, Mixed Tate motives, Ann. Math. 140 (1994), 557-605.

[2] F. Brown, The massless higher-loop two point function, Commun. Math. Phys. 287 (2009), 925-958.

[3] K.-T. Chen, Iterated path integrals, Bull. Amer. Math. Soc. 83(5) (1977), 831-879.

[4] A. Connes and D. Kreimer, Hopf algebras, renormalization and non-commtative geometry, Commun. Math. Phys. 199 (1998), 203-242, arXiv:hep-th/9808042v1.

[5] C. Duhr, Hopf algebras, coproducts and symbols: an application to Higgs boson amplitudes, arXiv:1203.0454v1 [hep-ph], 2012.

[6] C. Duhr, H. Gangl and J. Rhodes, From polylogs and symbols to polylogarithmic functions, arXiv:1110.0458v1 [math-ph], 2011. 
[7] H. Gangl, A.B. Goncharov and A. Levin, Multiple polylogarithms, polygons, trees and algebraic cycles, Symposia in Pure Mathematics 80(2) (2009), arXiv.org/abs/math/0508066v1.

[8] A.B. Goncharov, Multiple polylogarithms, cyclotomy and modular complexes, Math Res. Lett. 5 (1998), 497-516, arXiv:1105.2076v1.

[9] _ Multiple polylogarithms and mixed tate motives, (2001), arXiv:math/0103059v4.

[10] _ Galois symmetries of fundamental groupoids and noncommutative geometry, Duke Math. J. 128 (2005), 209-284, arXiv:math/0208144v4.

[11] A.B. Goncharov, M. Spradlin, C. Vergu and A. Volovich, Classical polylogarithms for amplitudes and wilson loops, Phys. Rev. Lett. 105(15) (2010), 151605.

[12] J.-L. Loday and B. Vallette, Algebraic operads, Springer, New York, 2012.

UNIVERSITY OF HAMBURG

Mathematics Department

Bundestrasse, 55

20146, HAMBURG

GERMANY

E-mail address: susama.agarwala@math.hamburg.edu

Received 11 September, 2012 